\title{
MUTUALLY CATALYTIC BRANCHING IN THE PLANE: UNIQUENESS
}

\author{
Donald A. DAWSON ${ }^{\mathrm{a}, 1}$, Klaus FLEISCHMANN ${ }^{\mathrm{b}, 2}$, \\ Leonid MYTNIK $^{\mathrm{c}, *, 3}$, Edwin A. PERKINS ${ }^{\mathrm{d}, 4}$, Jie XIONG ${ }^{\mathrm{e}, 5}$ \\ ${ }^{\text {a }}$ School of Mathematics and Statistics, Carleton University, Ottawa, Canada K1S 5B6 \\ ${ }^{\mathrm{b}}$ Weierstrass Institute for Applied Analysis and Stochastics, Mohrenstr. 39, 10117 Berlin, Germany \\ ${ }^{\mathrm{c}}$ Faculty of Industrial Engineering and Management, Technion-Israel Institute of Technology, \\ Haifa 32000, Israel \\ ${ }^{\mathrm{d}}$ The University of British Columbia, Department of Mathematics, 1984 Mathematics Road, \\ Vancouver, B.C., Canada V6T 1Z2 \\ ${ }^{\mathrm{e}}$ University of Tennessee, Department of Mathematics, Knoxville, TN 37996-1300, USA
}

Received 16 February 2001, revised 27 November 2001

ABSTRACT. - We study a pair of populations in $\mathbb{R}^{2}$ which undergo diffusion and branching. The system is interactive in that the branching rate of each type is proportional to the local density of the other type. Previous work had established the existence of such a process and derived some of its small scale and large scale properties. This paper is primarily focused on the proof of uniqueness of solutions to the martingale problem associated with the model. The self-duality property of solutions, which is crucial for proving uniqueness and was used in the previous work to derive many of the qualitative properties of the process, is also established.

\footnotetext{
* Corresponding author.

E-mail addresses: ddawson@math.carleton.ca (D.A. Dawson), fleischmann@wias-berlin.de (K. Fleischmann), leonid@ie.technion.ac.il (L. Mytnik), perkins@math.ubc.ca (E.A. Perkins), jxiong@math.utk.edu (J. Xiong).

URLs: http://www.fields.utoronto.ca/dawson.html (D.A. Dawson), http://www.wias-berlin.de/ fleischm (K. Fleischmann), http://ie.technion.ac.il/leonid.phtml (L. Mytnik), http://www.math.ubc.ca/ perkins/perkins.html (E.A. Perkins), http://www.math.utk.edu/ jxiong/ (J. Xiong).

${ }^{1}$ Supported in part by an NSERC grant and a Max Planck Award.

${ }^{2}$ Supported in part by the DFG.

${ }^{3}$ Supported in part by the US-Israel Binational Science Foundation (grant No. 2000065) and the Israel Science Foundation (grant No. 116/01-10.0).

${ }^{4}$ Supported in part by an NSERC grant.

${ }^{5}$ Supported in part by UT-ORNL Science Alliance.
} 
(C) 2003 Éditions scientifiques et médicales Elsevier SAS

$M S C$ : primary 60K35; secondary 60G57, 60J80

Keywords: Catalytic super-Brownian motion; Collision local time; Martingale problem; Duality; Uniqueness; Markov property

RÉSUMÉ. - On étudie un couple de populations dans le plan, sujettes à des phénomènes de branchement et de diffusion.

Le taux de branchement de chaque type est supposé proportionnel à ka densité de l'autre type. L'existence et les propriétés à grande et petite échelle d'un tel processus a été établie dans un article précédent. Ce travail est centré sur l'unicité de la solution du problème des martingales associé au modèle.

La propriété d'auto-dualité, qui est cruciale dans la démonstration de l'unicité et a été employée dans le travail précédent pour établir de nombreuses propriétés qualitatives du processus, est également démontrée.

○ 2003 Éditions scientifiques et médicales Elsevier SAS

\section{Introduction and statement of results}

\subsection{Background, motivation and brief description of results}

Super-Brownian motion is a measure-valued process which arises as a limit of branching particle systems undergoing Brownian motion and critical (or asymptotically critical) branching. For example let us take large population of $N$ particles in $\mathbb{R}^{d}$ with small masses of $N^{-1}$ per particle and let them move as independent Brownian motions with diffusion rate $\sigma^{2}$. Suppose that each particle dies independently of the others with rate $N \rho(x)$ at site $x$ at time $t$ and at the time of death each particle (independently of the others) is replaced by 2 particles or by nothing with probability $1 / 2$ to each event. The replacement particles, if there are any, perform independent Brownian motions and the story of alternating branching and diffusions continues. If we define measure-valued process $X^{N}$ by

$$
X_{t}^{N}(A)=\{\text { mass of the particles alive at time } t \text { in } A\}, \quad \forall A \in \mathcal{B}\left(\mathbb{R}^{d}\right),
$$

then as $N$ goes to infinity the resulting $X^{N}$ converges in an appropriate topology to the measure-valued process known as super-Brownian motion with diffusion rate $\sigma^{2}$ and branching rate $\rho$. In dimension $d=1$ super-Brownian motion takes values in the space of measures which are absolutely continuous with respect to Lebesgue measure and its density satisfies the following stochastic partial differential equation (see $[9,15])$ :

$$
\frac{\partial X_{t}(x)}{\partial t}=\frac{\sigma^{2}}{2} \Delta X_{t}(x)+\sqrt{\rho(x) X_{t}(x)} \dot{W}_{t}(x), \quad(t, x) \in \mathbb{R}_{+} \times \mathbb{R},
$$

where $\Delta$ is the one-dimensional Laplacian and $\dot{W}$ is standard time-space white noise on $\mathbb{R}_{+} \times \mathbb{R}$. In dimensions $d \geqslant 2$ the super-Brownian motion takes values in the space of singular measures, therefore it can not be represented as a solution to the above SPDE (for discussion on parabolic SPDEs see e.g. [16]). It is characterized as a solution to an 
appropriate martingale problem (see e.g. Chapter 6 in [2]). One of the strongest tools available in the study of superprocesses is its Laplace transform. Write $\langle\mu, \varphi\rangle$ or $\mu(\varphi)$ to denote the integral of $\varphi$ with respect to a measure $\mu$. Then for the super-Brownian motion $X$ adapted to filtration $\mathcal{F}_{t}$ we have

$$
P\left[\mathrm{e}^{-\left\langle X_{t}, \varphi\right\rangle} \mid \mathcal{F}_{0}\right]=\mathrm{e}^{-\left\langle X_{0}, V_{t}(\varphi)\right\rangle}, \quad \forall \varphi \geqslant 0,
$$

where $V_{t}(\varphi)$ solves the following nonlinear partial differential equation:

$$
\left\{\begin{array}{l}
\frac{\partial v_{t}(x)}{\partial t}=\frac{\sigma^{2}}{2} \Delta v_{t}(x)-\frac{\rho(x)}{2} v_{t}(x)^{2}, \\
v_{0}=\varphi
\end{array}\right.
$$

This formula for Laplace transform is the key for proving that super-Brownian motion is a unique solution to the martingale problem and so is strong Markov.

Recently there has been considerable interest in the area of the superprocesses with interactions. In [5] solutions to the following system of stochastic partial differential equations were studied:

$$
\frac{\partial X_{t}^{i}(x)}{\partial t}=\frac{\sigma^{2}}{2} \Delta X_{t}^{i}(x)+\sqrt{\gamma X_{t}^{1}(x) X_{t}^{2}(x)} \dot{W}_{t}^{i}(x), \quad(t, x) \in \mathbb{R}_{+} \times \mathbb{R}, i=1,2,
$$

where $\dot{W}^{1}, \dot{W}^{2}$ are independent space time white noises and $\gamma>0$. In this model, called the mutually catalytic branching model, there are two types of particles each of which may branch only in the presence of the other type. More precisely the branching rate of each type at a site is proportional to the density of the other type present at that site.

A number of approaches are used to study uniqueness in law of interactive superprocesses (see e.g. the use of "historical calculus" in $[13,14,8]$, or exchangeable particle representation in [6]). For the one-dimensional mutually catalytic branching model (1.4), uniqueness was resolved in [12], by deriving the so-called exponential selfduality formula. We will introduce this formula now. Here and elsewhere we identify non-negative functions $X(x)$ which are integrable on compact sets with Radon measures $X(x) d x \equiv X(d x)$. If $\left(X^{1}, X^{2}\right)$ and $\left(\widetilde{X}^{1}, \widetilde{X}^{2}\right)$ are two independent solutions to (1.4) starting at $\left(X_{0}^{1}, X_{0}^{2}\right)$ and $\left(\widetilde{X}_{0}^{1}, \widetilde{X}_{0}^{2}\right)$ respectively (suppose that the initial conditions are in the space of continuous functions) then the self-duality formula states that

$$
\begin{aligned}
& P\left[\exp \left\{-\left\langle X_{t}^{1}+X_{t}^{2}, \widetilde{X}_{0}^{1}+\widetilde{X}_{0}^{2}\right\rangle+i\left\langle X_{t}^{1}-X_{t}^{2}, \widetilde{X}_{0}^{1}-\widetilde{X}_{0}^{2}\right\rangle\right\}\right] \\
& \quad=\widetilde{P}\left[\exp \left\{-\left\langle X_{0}^{1}+X_{0}^{2}, \widetilde{X}_{t}^{1}+\widetilde{X}_{t}^{2}\right\rangle+i\left\langle X_{0}^{1}-X_{0}^{2}, \widetilde{X}_{t}^{1}-\widetilde{X}_{t}^{2}\right\rangle\right\}\right] .
\end{aligned}
$$

In addition to proving the uniqueness result, the above self-duality formula is the key tool for deriving the long time behavior of the processes with infinite initial conditions through the long time behavior of the processes with finite initial conditions.

The question of extending the mutually catalytic branching model to dimensions greater than one was of interest for a number of reasons. One's intuition does not work very well in this case (see, for example, the intuitive "non-existence" argument in the introduction of [3]). Eventually the existence of the model in dimension $d=2$ was proved in $[3,4]$, providing $\gamma / \sigma^{2}$ is small enough. More specifically, it was shown 
that a mutually catalytic process $\mathbf{X}=\left(X^{1}, X^{2}\right)$ makes sense in dimension $d=2$ as a pair of measure-valued processes that solves the following martingale problem. For an appropriate class of test functions $\varphi_{i}, i=1,2$,

$$
M_{t}^{i}\left(\varphi_{i}\right)=\left\langle X_{t}^{i}, \varphi_{i}\right\rangle-\left\langle X_{0}^{i}, \varphi_{i}\right\rangle-\int_{0}^{t}\left\langle X_{s}^{i}, \frac{\sigma^{2} \Delta \varphi_{i}}{2}\right\rangle d s, \quad t \geqslant 0, i=1,2
$$

are orthogonal continuous $L^{2}$-martingales such that $M_{0}^{i}\left(\varphi_{i}\right)=0$ and

$$
\left\langle M^{i}\left(\varphi_{i}\right)\right\rangle_{t}=\gamma\left\langle L_{\mathbf{X}}(t), \varphi_{i}^{2}\right\rangle, \quad t \geqslant 0, i=1,2 .
$$

Here $L_{\mathbf{X}}$ is the collision local time of $X^{1}$ and $X^{2}$ loosely described by

$$
L_{\mathbf{X}}(t, d x) \equiv \int_{0}^{t} \int_{\mathbb{R}^{2}} \delta_{x}(y) X_{s}^{1}(d x) X_{s}^{2}(d y) d s, \quad t \geqslant 0,
$$

whereas the precise definition is given in Definition 1.1 below. [3] deals with the finite measures state space and [4] handles the infinite measures case. Several interesting properties of mutually catalytic process were derived in these papers, such as absolute continuity at fixed times, segregation of types property, the extinction of one type as time goes to infinity and a number of others. The proof of some of these properties is based on self-duality formula which we are going to derive in this paper under additional integrability condition (IntC). The uniqueness for the mutually catalytic martingale problem, which is the major result of this work, follows from the self-duality formula. The derivation of self-duality formula in $d=2$ is by no means simple generalization of $d=1$ case. The main problem is unboundedness of the densities of the processes. To circumvent this problem, first, we introduce an additional integrability condition (IntC) and prove self-duality, and hence uniqueness, under this condition. Second, to show that our uniqueness result is not vacuous, we verify existence of the processes satisfying (IntC). Some moment calculations are required to show that the processes with finite initial conditions constructed in [3] satisfy (IntC), and hence are unique solutions to the corresponding martingale problem. Note that these moment calculations are based on moment duality introduced in [3]. Besides uniqueness, we prove the strong Markov property for the finite measure case-the requirement of a side condition like (IntC) means, there is a bit to say here. Moreover, (IntC) also allows us to give a simple proof of absolute continuity of collision local time with respect to time, that is, the existence of a collision measure process $K_{\mathbf{X}}(t, \cdot)$ such that $L_{\mathbf{X}}(d t, \cdot)=K_{\mathbf{X}}(t, \cdot) d t$.

In the case of infinite measures initial conditions, the situation is more complicated. We were able to derive (IntC), and hence self-duality and uniqueness, only for initial conditions with bounded densities. As the problem of finding a proper state space for these infinite measure-valued processes remains unresolved, we do not have results on the Markov and strong Markov properties in this case. 


\subsection{Notation}

We will try to use the same notation as in [3] and [4].

We use $c$ to denote a positive (finite) constant which may vary from place to place. A $c$ with some additional subscript or superscript usually denotes a specific constant. Write $|\cdot|$ for the Euclidean norm in $\mathbb{R}^{d}, d \geqslant 1$.

For $\lambda \in \mathbb{R}$ introduce the reference function $\phi_{\lambda}$ :

$$
\phi_{\lambda}(x) \equiv \mathrm{e}^{-\lambda|x|}, \quad x \in \mathbb{R}^{d} .
$$

For $f: \mathbb{R}^{d} \mapsto \mathbb{R}$ put

$$
|f|_{\lambda} \equiv \sup _{x \in \mathbb{R}^{d}}|f(x)| / \phi_{\lambda}(x), \quad \lambda \in \mathbb{R}
$$

and

$$
\|f\|_{\infty} \equiv \sup _{x \in \mathbb{R}^{d}}|f(x)| .
$$

For $\lambda \in \mathbb{R}$, let $\mathcal{B}_{\lambda}=\mathcal{B}_{\lambda}\left(\mathbb{R}^{2}\right)$ denote the set of all measurable (real-valued) functions $f$ such that $|f|_{\lambda}$ is finite. Introduce the spaces

$$
\begin{aligned}
& \mathcal{B}_{\text {tem }}=\mathcal{B}_{\text {tem }}\left(\mathbb{R}^{2}\right) \equiv \bigcap_{\lambda>0} \mathcal{B}_{-\lambda}, \quad \mathcal{B}_{\text {exp }}=\mathcal{B}_{\text {exp }}\left(\mathbb{R}^{2}\right) \equiv \bigcup_{\lambda>0} \mathcal{B}_{\lambda}, \\
& \mathcal{B}_{\text {rap }}=\mathcal{B}_{\text {rap }}\left(\mathbb{R}^{2}\right) \equiv \bigcap_{\lambda>0} \mathcal{B}_{\lambda}
\end{aligned}
$$

of tempered, exponentially decreasing and rapidly decreasing functions respectively. Also let $\mathcal{B}=\mathcal{B}\left(\mathbb{R}^{2}\right)$ (respectively $\mathcal{B}_{\mathrm{b}}=\mathcal{B}_{\mathrm{b}}\left(\mathbb{R}^{2}\right), \mathcal{B}_{\mathrm{b}, \text { com }}=\mathcal{B}_{\mathrm{b} \text {, com }}\left(\mathbb{R}^{2}\right) \mathcal{C}=\mathcal{C}\left(\mathbb{R}^{2}\right), \mathcal{C}_{\mathrm{b}}=$ $\mathcal{C}_{\mathrm{b}}\left(\mathbb{R}^{2}\right), \mathcal{C}_{\text {com }}=\mathcal{C}_{\text {com }}\left(\mathbb{R}^{2}\right)$ ) be the set of all measurable (respectively bounded measurable, bounded measurable with compact support, continuous, bounded continuous, continuous with compact support) functions on $\mathbb{R}^{2}$. Note that $\mathcal{B}$ will also serve as a notation for Borel sets in $\mathbb{R}^{2}$.

Let $\mathcal{C}_{\lambda}$ refer to the set of continuous $f$ in $\mathcal{B}_{\lambda}$ with the additional property that the $f(x) / \phi_{\lambda}(x)$ has a finite limit as $|x| \uparrow \infty$. The definition of $\mathcal{C}_{\text {tem }}, \mathcal{C}_{\text {exp }}$ and $\mathcal{C}_{\text {rap }}$ is analogous to that of $\mathcal{B}_{\text {tem }}, \mathcal{B}_{\text {exp }}$ and $\mathcal{B}_{\text {rap }}$. If $F$ is any subset of continuous functions on $\mathbb{R}^{d}$ then $F^{(m)}$ (respectively $F^{\infty}$ ) is a subset of functions in $F$ whose partial derivatives up to the order $m$ (respectively of any order) belong to $F$. For example $\mathcal{C}_{\text {com }}^{\infty}$ is the subspace of infinitely differentiable functions in $\mathcal{C}_{\text {com }}$.

If $F$ is a set of functions we will write $F_{+}$or $F^{+}$for non-negative functions in $F$.

The topology on $\mathcal{C}_{\text {tem }}$ is induced by the metric

$$
d_{\mathrm{tem}}(f, g) \equiv \sum_{n=1}^{\infty} 2^{-n}\left(|f-g|_{-1 / n} \wedge 1\right), \quad f, g \in \mathcal{C}_{\mathrm{tem}} .
$$

Let $\mathcal{M}$ be the set of all Radon measures on $\mathbb{R}^{2}$. Then let $\mathcal{M}_{\mathrm{tem}}=\mathcal{M}_{\mathrm{tem}}\left(\mathbb{R}^{2}\right)$ denote the subset of all measures $\mu$ in $\mathcal{M}$ such that $\left\langle\mu, \phi_{\lambda}\right\rangle<\infty$, for all $\lambda>0$. We topologize 
the set of tempered measures $\mathcal{M}_{\text {tem }}$ by the metric

$$
d_{\mathrm{tem}}(\mu, \nu) \equiv d_{0}(\mu, \nu)+\sum_{n=1}^{\infty} 2^{-n}\left(|\mu-v|_{1 / n} \wedge 1\right), \quad \mu, v \in \mathcal{M}_{\mathrm{tem}} .
$$

Here $d_{0}$ is a complete metric on the space of Radon measures on $\mathbb{R}^{2}$ inducing the vague topology, and $|\mu-v|_{\lambda}$ is abbreviation for $\left|\left\langle\mu, \phi_{\lambda}\right\rangle-\left\langle v, \phi_{\lambda}\right\rangle\right|$. Note that $\left(\mathcal{M}_{\mathrm{tem}}, d_{\mathrm{tem}}\right)$ is a Polish space and $\mu_{n} \rightarrow \mu$ in $\mathcal{M}_{\text {tem }}$ if and only if $\left\langle\mu_{n}, \varphi\right\rangle \rightarrow\langle\mu, \varphi\rangle$ for all $\varphi \in \mathcal{C}_{\text {exp }}$. Let $\mathcal{M}_{\mathrm{f}}=\mathcal{M}_{\mathrm{f}}\left(\mathbb{R}^{2}\right)$ denote the space of finite measures on $\mathbb{R}^{2}$ with the topology of weak convergence. Let $\mathcal{M}_{\text {rap }}$ be the space of rapidly decreasing measures $\mu$ on $\mathbb{R}^{2}$ such that $\langle\mu, \varphi\rangle<\infty$ for any $\varphi \in \mathcal{B}_{\text {tem }}$. We say that $\mu_{n} \Rightarrow \mu$ in $\mathcal{M}_{\text {rap }}$ if $\mu_{n} \Rightarrow \mu$ in $\mathcal{M}_{\mathrm{f}}$ and $\sup _{n}\left\langle\mu_{n}, \phi_{\lambda}\right\rangle<\infty$ for any $\lambda<0$.

For any metric space $E$ let $D\left(\mathbb{R}_{+}, E\right)$ (respectively $C\left(\mathbb{R}_{+}, E\right)$ ) be the space of cadlag (respectively continuous) $E$-valued functions with Skorohod topology. Let $\mathcal{M}_{1}(E)$ denote the set of probability measures on $E$ and $\mathcal{B}(E)$ serve as a notation for Borel sets in $E$.

Let ${ }^{\varepsilon} \mathrm{p}_{t}(x) \equiv \varepsilon^{-2} P\left({ }^{\varepsilon} \xi_{t}=x \mid{ }^{\varepsilon} \xi_{0}=\mathbf{0}\right), t>0, x \in \varepsilon \mathbb{Z}^{2}$, where ${ }^{\varepsilon} \xi_{t}$ is a continuous time simple random walk which jumps to a nearest neighbor in $\varepsilon \mathbb{Z}^{2}$ with rate $2 \varepsilon^{-2} \sigma^{2}$. Write $\left(\xi, \Pi_{x}, x \in \mathbb{R}^{2}\right)$ for the Brownian motion on $\mathbb{R}^{2}$ with variance parameter $\sigma^{2}$,

$$
\mathrm{p}_{t}(x, y)=\left(2 \pi \sigma^{2} t\right)^{-1} \exp \left\{-|x-y|^{2} / 2 t \sigma^{2}\right\}, \quad t>0, x, y \in \mathbb{R}^{2},
$$

for its transition density, and $\left\{S_{t}: t \geqslant 0\right\}$ for the corresponding semigroup. With slight abuse of notation let $\mathrm{p}_{t}(x) \equiv \mathrm{p}_{t}(x, 0)$. If $\mu \in \mathcal{M}_{\mathrm{tem}}$, set $S_{t} \mu(x) \equiv \int \mathrm{p}_{t}(x-y) \mu(d y)$.

For $E$ a topological space let $\mathcal{L}(X)$ be the law on $E$ of $E$-valued random variable $X$.

\subsection{Uniqueness theorems}

In this subsection we will state our main uniqueness theorems. We start with necessary definitions. Let $\mathbf{X}=\left(X^{1}, X^{2}\right)$ denote an $\mathcal{M}_{\text {tem }}^{2}$-valued process, where $\mathcal{M}_{\text {tem }}^{2}=\mathcal{M}_{\text {tem }} \times$ $\mathcal{M}_{\text {tem }}$. Define a pair of measures on the plane by

$$
\begin{gathered}
L_{\mathbf{X}}^{*, \delta}(t, d x) \equiv \frac{1}{\delta} \int_{0}^{\delta} \int_{0}^{t} S_{r} X_{s}^{1}(x) S_{r} X_{s}^{2}(x) d s d r d x, \quad t \geqslant 0, \delta>0, \\
L_{\mathbf{X}}^{\delta}(t, d x) \equiv \int_{0}^{t} S_{\delta} X_{s}^{1}(x) S_{\delta} X_{s}^{2}(x) d s d x, \quad t \geqslant 0, \delta>0 .
\end{gathered}
$$

Definition 1.1 (Collision local time). - Let $\mathbf{X}=\left(X^{1}, X^{2}\right)$ be an $\mathcal{M}_{\mathrm{tem}}^{2}$-valued continuous process. The collision local time of $\mathbf{X}$ (if it exists) is a continuous nondecreasing $\mathcal{M}_{\mathrm{tem}}$-valued stochastic process $t \mapsto L_{\mathbf{X}}(t)=L_{\mathbf{X}}(t, \cdot)$ such that

$$
\left\langle L_{\mathbf{X}}^{*, \delta}(t), \varphi\right\rangle \rightarrow\left\langle L_{\mathbf{X}}(t), \varphi\right\rangle \quad \text { as } \delta \downarrow 0 \text { in probability, }
$$

for all $t>0$ and $\varphi \in \mathcal{C}_{\text {com }}$. 
The collision local time will be also considered as a locally finite measure $L_{\mathbf{X}}(d s, d x)$ on $\mathbb{R}_{+} \times \mathbb{R}^{2}$.

Now we are ready to introduce the martingale problem for the mutually catalytic model in $d=2$. Note that all filtrations will be assumed to be right continuous and contain the null sets at time 0 .

DEFINITION 1.2 (Martingale problem $(\mathbf{M P})_{\mathbf{X}_{0}}^{\sigma, \gamma}$ ). - A continuous $\mathcal{F}_{t}$-adapted $\mathcal{M}_{\text {tem }}^{2}$ valued process $\mathbf{X}=\left(X^{1}, X^{2}\right)$ on some $\left(\Omega, \mathcal{F}, \mathcal{F}_{t}, P\right)$ satisfies $(\mathbf{M P})_{\mathbf{X}_{0}}^{\sigma, \gamma}$ if and only if $\forall \varphi_{i} \in \mathcal{C}_{\text {exp }}^{(2)}, i=1,2$,

$$
M_{t}^{i}\left(\varphi_{i}\right)=\left\langle X_{t}^{i}, \varphi_{i}\right\rangle-\left\langle X_{0}^{i}, \varphi_{i}\right\rangle-\int_{0}^{t}\left\langle X_{s}^{i}, \frac{\sigma^{2} \Delta \varphi_{i}}{2}\right\rangle d s, \quad t \geqslant 0, i=1,2
$$

are orthogonal continuous $L^{2} \mathcal{F}_{t}$-martingales, such that $M_{0}^{i}\left(\varphi_{i}\right)=0$ and

$$
\left\langle M^{i}\left(\varphi_{i}\right)\right\rangle_{t}=\gamma\left\langle L_{\mathbf{X}}(t), \varphi_{i}^{2}\right\rangle, \quad t \geqslant 0, i=1,2 .
$$

Note that $\mathbf{X}_{0}$ may be a random $\mathcal{F}_{0}$-measurable initial condition.

To present the results dealing with solutions to the above martingale problem we need to define spaces of measures satisfying some regularity conditions.

DEFINITION 1.3. - Define

$$
\begin{gathered}
\overline{\mathrm{p}}_{t}\left(\mu_{1}, \mu_{2}\right)(x) \equiv S_{t} \mu_{1}(x) S_{t} \mu_{2}(x), \quad t>0, \\
\bar{g}_{t}\left(\mu_{1}, \mu_{2}\right)(x) \equiv \int_{0}^{t} S_{s} \mu_{1}(x) S_{s} \mu_{2}(x) d s=\int_{0}^{t} \overline{\mathrm{p}}_{s}\left(\mu_{1}, \mu_{2}\right)(x) d s, \quad t>0 .
\end{gathered}
$$

Definition 1.4. - Write $\mu=\left(\mu^{1}, \mu^{2}\right) \in \mathcal{M}_{\mathrm{f}, \mathrm{e}}$ and say that $\mu$ satisfies the energy condition if and only if $\mu \in \mathcal{M}_{\mathrm{f}}^{2} \equiv \mathcal{M}_{\mathrm{f}} \times \mathcal{M}_{\mathrm{f}}$ and

$$
\int_{\mathbb{R}^{2}} \bar{g}_{t}\left(\mu_{1}, \mu_{2}\right)(x) d x<\infty, \quad \forall 0<t<\infty .
$$

Write $\mu=\left(\mu^{1}, \mu^{2}\right) \in \mathcal{M}_{\mathrm{f}, \mathrm{se}}$ and say that $\mu$ satisfies the strong energy condition if and only if $\mu \in \mathcal{M}_{\mathrm{f}}^{2}$ and $\forall p \in(0,1]$ there exists $c=c(p, \mu)$ such that for all $t>0$

$$
\max _{1 \leqslant i, j \leqslant 2} \int_{\mathbb{R}^{2}} \overline{\mathrm{p}}_{t}\left(\mu_{i}, \mu_{j}\right)(x) d x \leqslant c t^{-p} .
$$

Remark 1.5. - In view of Lemma 8(b) of [3] (see also (1.8) below) the strong energy condition need only be checked for $0<t<1$ (as $c t^{-1} \leqslant c t^{-p}$ for $t \geqslant 1$ ). As we only need to check $t=2^{-n}$ and $p=p_{n} \downarrow 0,(n \in \mathbb{N})$, clearly $\mathcal{M}_{\mathrm{f}, \mathrm{e}}$ is a Borel subset of $\mathcal{M}_{\mathrm{f}}^{2}$. 
Definition 1.6. - Write $\mu=\left(\mu^{1}, \mu^{2}\right) \in \mathcal{M}_{\text {tem,e }}$ and say that $\mu$ satisfies the energy condition if and only if $\mu \in \mathcal{M}_{\mathrm{tem}}^{2}$ and

$$
\int_{\mathbb{R}^{2}} \bar{g}_{t}\left(\mu_{1}, \mu_{2}\right)(x) \phi_{\lambda}(x) d x<\infty, \quad \forall \lambda>0,0<t<\infty .
$$

Write $\mu=\left(\mu^{1}, \mu^{2}\right) \in \mathcal{M}_{\text {tem,se }}$ and say that $\mu$ satisfies the strong energy condition if and only if $\mu \in \mathcal{M}_{\text {tem }}^{2}$ and for any $p \in(0,1)$ and $\lambda>0$ there is $c=c(p, \lambda, \mu)$ such that for any $t>0$

$$
\max _{1 \leqslant i, j \leqslant 2} \int_{\mathbb{R}^{2}} \overline{\mathrm{p}}_{t}\left(\mu_{i}, \mu_{j}\right)(x) \phi_{\lambda}(x) d x<c t^{-p} .
$$

Definition 1.7. - Write $\mu=\left(\mu^{1}, \mu^{2}\right) \in \mathcal{M}_{\text {rap,e }}$ and say that $\mu$ satisfies the energy condition if and only if $\mu \in \mathcal{M}_{\text {rap }}^{2}$ and

$$
\int_{\mathbb{R}^{2}} \bar{g}_{t}\left(\mu_{1}, \mu_{2}\right)(x) \phi_{-\lambda}(x) d x<\infty, \quad \forall \lambda>0,0<t<\infty .
$$

In Theorem 11(a) of [3] solutions to (MP) ${ }_{\mathbf{X}_{0}}^{\sigma, \gamma}$ were constructed for $\mathbf{X}_{0}$, a deterministic initial condition in $\mathcal{M}_{\mathrm{f}, \mathrm{e}}$, providing

$$
\gamma / \sigma^{2}<\left(3 \sqrt{6} \pi c_{\mathrm{rw}}\right)^{-1}
$$

where $c_{\mathrm{rw}}$ is a universal constant (independent of $\sigma^{2}$ ) defined in Lemma 8(b) of [3] by

$$
c_{\mathrm{rw}} \equiv \sup _{x \in \varepsilon \mathbb{Z}^{2}, t \geqslant 0}{ }^{\varepsilon} \mathrm{p}_{t}(x) t \sigma^{2}
$$

In Theorem 4 of [4] solutions to $(\mathbf{M P})_{\mathbf{X}_{0}}^{\sigma, \gamma}$ were constructed for infinite initial conditions $\mathbf{X}_{0} \in \mathcal{M}_{\text {tem }}^{2}$ which have densities $\left(x_{0}^{1}, x_{0}^{2}\right)$ in $\mathcal{B}_{\text {tem }}^{2}$. Recall that we often use the same letter to denote a measure and its density.

In order to establish uniqueness in law of solutions to $(\mathbf{M P})_{\mathbf{X}_{0}}^{\sigma, \gamma}$, we need to assume additional integrability condition:

For any compact $K \subset \mathbb{R}^{2}$ set

$$
H_{\varepsilon, K}\left(\mathbf{X}_{s}\right)=\int_{K} \int_{K}\left(1+|x-y|^{-1}\right) S_{\varepsilon} X_{s}^{1}(x) S_{\varepsilon} X_{s}^{2}(x) S_{\varepsilon} X_{s}^{1}(y) S_{\varepsilon} X_{s}^{2}(y) d x d y, \quad \varepsilon>0 .
$$

(IntC) For each $0<\delta<T<\infty$, compact $K \subset \mathbb{R}^{2}$,

$$
P\left[\int_{\delta}^{T} H_{\varepsilon, K}\left(\mathbf{X}_{s}\right) d s \mid \mathcal{F}_{\delta}\right] \text { is bounded in probability as } \varepsilon \downarrow 0
$$

i.e. $\forall \eta>0 \exists M>0$ such that $\limsup _{\varepsilon \downarrow 0} P\left(P\left[\int_{\delta}^{T} H_{\varepsilon, K}\left(\mathbf{X}_{s}\right) d s \mid \mathcal{F}_{\delta}\right]>M\right)<\eta$. 
Note that (IntC) is implied by the simpler condition

(SIntC)

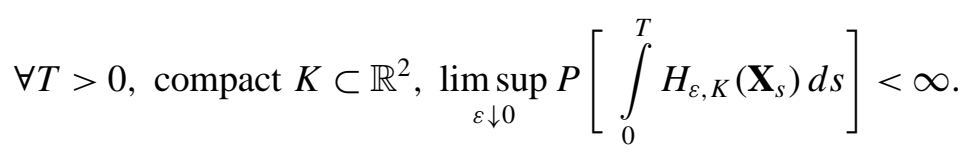

We now introduce an integrability condition on possibly random initial condition $\mathbf{X}_{0}$ :

(EnC)

$$
P\left[\sum_{j=1}^{2} X_{0}^{j}\left(\phi_{\lambda}\right)^{2}+\int_{\mathbb{R}^{2}} \bar{g}_{t}\left(X_{0}^{1}, X_{0}^{2}\right)(x) \phi_{\lambda}(x) d x\right]<\infty, \quad \forall t>0, \forall \lambda>0 .
$$

Now we are ready to present our main result.

THEOREM 1.8 (General uniqueness theorem). - Assume $\gamma / \sigma^{2}<\left(\sqrt{6} \pi c_{\mathrm{rw}}\right)^{-1}$ and let $\mathbf{X}_{0} \in \mathcal{M}_{\mathrm{tem}, \mathrm{e}}$ be a possibly random initial condition satisfying (EnC). Then there is at most one solution to $(\mathbf{M P})_{\mathbf{X}_{0}}^{\sigma, \gamma}$ satisfying (IntC).

Notation. - Let $\Omega_{0} \equiv C\left(\mathbb{R}_{+}, \mathcal{M}_{\mathrm{f}}^{2}\right), \Omega_{\text {rap }} \equiv C\left(\mathbb{R}_{+}, \mathcal{M}_{\text {rap }}^{2}\right), \Omega_{\text {tem }} \equiv C\left(\mathbb{R}_{+}, \mathcal{M}_{\text {tem }}^{2}\right)$ with the usual topology of uniform convergence on compact subsets of $\mathbb{R}_{+}$.

It will be shown in Theorem 5.1 of Section 5.1 that the solutions constructed in [3] with $\mathbf{X} \in \mathcal{M}_{\mathrm{f}, \mathrm{e}}$ satisfy (IntC). It was also shown in [3] that $\mathbf{X}_{t} \in \mathcal{M}_{\mathrm{f}, \mathrm{e}}$ for all $t>0$ a.s. and this allows us to show the strong Markov property for the processes starting from finite initial conditions and satisfying (IntC). Overall we have the following theorem.

THEOREM 1.9 (Finite measure initial conditions). - Assume $\gamma / \sigma^{2}<\left(3 \sqrt{6} \pi c_{\mathrm{rw}}\right)^{-1}$ and $\mathbf{X}_{0} \in \mathcal{M}_{\mathrm{f}, \mathrm{e}}$.

(a) There is a process $\mathbf{X}$ satisfying the martingale problem $(\mathbf{M P})_{\mathbf{X}_{0}}^{\sigma, \gamma}$ and the integrability condition (IntC), and such that $\mathbf{X}_{t} \in \mathcal{M}_{\mathrm{f}, \mathrm{e}}$ for any $t \geqslant 0$ a.s. If $\mathbf{X}_{0} \in \mathcal{M}_{\mathrm{f}, \text { se }}$ then there is a solution to (MP) $)_{\mathbf{X}_{0}, \gamma}^{\sigma, \gamma}$ satisfying (SIntC).

(b) The law $P_{\mathbf{X}_{0}}$ on $\Omega_{0}$ of the solution in (a) is unique.

(c) There is a time-homogeneous Borel Markov transition kernel $\mathrm{P}=\left\{\mathrm{P}_{t}(\mu, d v)\right.$ : $\left.t>0, \mu \in \mathcal{M}_{\mathrm{f}, \mathrm{e}}\right\}$ on $\mathcal{M}_{\mathrm{f}, \mathrm{e}}$ such that any process satisfying (MP) $)_{\mathbf{X}_{0}, \gamma}^{\sigma, \gamma}$ and (IntC) on $\left(\Omega, \mathcal{F}, \mathcal{F}_{t}, P\right)$ is $\left(\mathcal{F}_{t}\right)$-strong Markov with transition kernel $\mathrm{P}$.

The martingale problem (MP) $)_{\mathbf{X}_{0}}^{\sigma, \gamma}$ for finite initial conditions was defined in [3] with a larger set of test functions than in Definition 1.2. Clearly any solution to the martingale problem $(\mathbf{M P})_{\mathbf{X}_{0}}^{\sigma, \gamma}$ in [3] is a solution to the $(\mathbf{M P})_{\mathbf{X}_{0}}^{\sigma, \gamma}$ of Definition 1.2. Therefore the existence part of (a) follows from the proof of Theorem 11(a) in [3]. To complete the proof of (a) we need to verify integrability conditions (IntC) and (SIntC) for the constructed processes. This will be accomplished in Section 5.1. Then part (b) will follow from part (a) and Theorem 1.8. Part (c) will be proved in Section 5.4.

Remark 1.10. - To ensure the existence of solutions to (MP) $\mathbf{\mathbf { X }}_{0}^{\sigma, \gamma}$ satisfying (IntC), condition (1.7) maybe weakened to $\gamma / \sigma^{2}<1 / \sqrt{6}$, although to ensure only existence (without (IntC)) it may be weakened to $\gamma / \sigma^{2}<2 / \sqrt{6}$ (see Remark 12(ii) of [3] and Theorem 5.1 of Section 5.1 deriving (IntC) below). 
The uniqueness in law of the mutually catalytic branching process $\mathbf{X}$ with $\mathbf{X}_{0} \in \mathcal{B}_{\mathrm{b}}^{2}$, constructed in [4], is established in the following theorem.

THEOREM 1.11 (Initial conditions with bounded densities). - Assume

$$
\gamma / \sigma^{2}<\left(\sqrt{6} \pi c_{\mathrm{rw}}\right)^{-1}
$$

and $\mathbf{X}_{0} \in\left(\mathcal{B}_{\mathrm{b}}^{+}\right)^{2}$. Then

(a) There is a solution to (MP) $)_{\mathbf{X}_{0}}^{\sigma, \gamma}$ satisfying (SIntC).

(b) The law $P_{\mathbf{X}_{0}}$ on $\Omega_{\text {tem }}$ of the solution in (a) is unique.

The existence of a solution to (MP) $\mathbf{\mathbf { X }}_{0}^{\sigma, \gamma}$ follows from Theorem 4 and Remark 9(i) of [4]. To complete the proof of (a) we need to verify integrability condition (SIntC) for the processes constructed in [4]. This will be accomplished in Section 2 in Corollary 2.11. Part (b) then follows immediately from part (a) and Theorem 1.8.

\subsection{Duality relation}

The key ingredient in the uniqueness argument is an exponential duality introduced in [12], [5] for solutions to the analogue of $(\mathbf{M P})_{\mathbf{X}_{0}}^{\sigma, \gamma}$ on $\mathbb{R}^{1}$ and on a lattice respectively. For our continuum setting the dual process $\widetilde{\mathbf{X}}$ will be a particular solution to $(\mathbf{M P})_{\mathbf{X}_{0}}^{\sigma, \gamma}$ constructed in [3], [4] for particularly nice initial conditions, which we now describe. Let $\tilde{x}_{0}^{j}(\cdot) \in \mathcal{B}_{\mathrm{b}}^{+}$and set $\widetilde{X}_{0}^{j}(d x)=\tilde{x}_{0}^{j}(x) d x, j=1,2$. For $\varepsilon>0$ define $\widetilde{X}_{0}^{j, \varepsilon}: \mathbb{Z}^{2} \mapsto[0, \infty)$ by

$$
\tilde{X}_{0}^{j, \varepsilon}\left(\varepsilon^{-1} x\right)=\varepsilon^{-2} \int_{C_{\varepsilon}(x)} \tilde{x}_{0}^{j}(y) d y, \quad j=1,2,
$$

where $C_{\varepsilon}(x)$ is the square of sidelength $\varepsilon$ and southwest corner $x \in \varepsilon \mathbb{Z}^{2}$. Let $\left\{W_{t}^{j}(x): x \in \mathbb{Z}^{2}, j=1,2\right\}$ be a collection of independent standard 1-dimensional Brownian motions on some filtered probability space and consider the unique (in law) solutions of

$$
\begin{gathered}
\tilde{X}_{t}^{j, \varepsilon}(x)=\widetilde{X}_{0}^{j, \varepsilon}(x)+\int_{0}^{t} \frac{\sigma^{2}}{2} \Delta \widetilde{X}_{s}^{j, \varepsilon}(x) d s+\int_{0}^{t} \sqrt{\gamma \widetilde{X}_{s}^{1, \varepsilon}(x) \widetilde{X}_{s}^{2, \varepsilon}(x)} d W_{s}^{j}(x), \\
x \in \mathbb{Z}^{2}, t \geqslant 0, j=1,2,
\end{gathered}
$$

constructed in [5]. Here ${ }^{1} \Delta$ is the usual discrete Laplacian on $\mathbb{Z}^{2}\left({ }^{1} \Delta f(x)=\sum_{i=1}^{2}(f(x+\right.$ $\left.\left.e_{i}\right)+f\left(x-e_{i}\right)-2 f(x)\right), e_{i}$ is the $i$ th unit basis vector). We then consider the rescaled process

$$
{ }^{\varepsilon} \widetilde{X}_{t}^{j}(x) \equiv \widetilde{X}_{t \varepsilon^{-2}}^{j, \varepsilon}\left(x \varepsilon^{-1}\right), \quad x \in \varepsilon \mathbb{Z}^{2}, t \geqslant 0
$$

and define its associated measure-valued process ${ }^{\varepsilon} \widetilde{\mathbf{X}} .=\left({ }^{\varepsilon} \widetilde{X}^{1},{ }^{\varepsilon} \widetilde{X}^{2}\right)$ by

$$
\left\langle\tilde{X}_{t}^{j}, \varphi\right\rangle=\sum_{x \in \varepsilon \mathbb{Z}^{2}}{ }^{\varepsilon} \widetilde{X}_{t}^{j}(x) \varphi(x) \varepsilon^{2}=\int_{\mathbb{R}^{2}}{ }^{\varepsilon} \tilde{X}_{t}^{j}(x) \varphi(x) d^{\varepsilon} x, \quad t \geqslant 0, j=1,2,
$$


where $d^{\varepsilon} x$ assigns mass $\varepsilon^{2}$ to each point in $\varepsilon \mathbb{Z}^{2}$. Let ${ }^{\varepsilon} \mathcal{M}_{\text {tem }}$ denote the subspace of $\mathcal{M}_{\text {tem }}$ of measures with densities with respect to $d^{\varepsilon} x$. Then ${ }^{\varepsilon} \widetilde{\mathbf{X}}$. is a ${ }^{\varepsilon} \mathcal{M}_{\text {tem }}^{2}$-valued process. Clearly ${ }^{\varepsilon} \widetilde{\mathbf{X}}_{0} \Rightarrow \widetilde{\mathbf{X}}_{0}$ in $\mathcal{M}_{\text {tem }}^{2}$. Propositions 37, 38 and Remark 9(i) of [4] show that if

$$
\gamma / \sigma^{2}<\left(\sqrt{6} \pi c_{\mathrm{rw}}\right)^{-1}
$$

then $\left\{\tilde{}{ }^{\varepsilon}: \varepsilon>0\right\}$ are tight in $C\left(\mathbb{R}_{+}, \mathcal{M}_{\text {tem }}^{2}\right)$ and any weak limit point $\widetilde{\mathbf{X}}=\left(\widetilde{X}^{1}, \widetilde{X}^{2}\right)$ (that is

$$
\varepsilon_{n} \widetilde{\mathbf{X}} \Rightarrow \widetilde{\mathbf{X}}
$$

for some $\varepsilon_{n} \downarrow 0$ ) satisfies (MP) ${\underset{\mathbf{\mathbf { X }}}{0}}_{\text {, }}^{\sigma, \gamma}$. Using notation from [4], we set

$$
\begin{aligned}
{ }^{\varepsilon} m_{t}^{1122}(\vec{x}) & \equiv{ }^{\varepsilon} m_{t}^{1122}\left(x_{1}, x_{2}, x_{3}, x_{4}\right) \\
& =\widetilde{P}\left({ }^{\varepsilon} \widetilde{X}_{t}^{1}\left(x_{1}\right)^{\varepsilon} \widetilde{X}_{t}^{1}\left(x_{2}\right)^{\varepsilon} \widetilde{X}_{t}^{2}\left(x_{3}\right)^{\varepsilon} \widetilde{X}_{t}^{2}\left(x_{4}\right)\right), \quad\left(\vec{x} \in\left(\varepsilon \mathbb{Z}^{2}\right)^{4}\right) .
\end{aligned}
$$

Then Corollary 31 of [4] states that if

$$
\gamma / \sigma^{2}<\frac{\sin (\pi(1-p))}{\sqrt{6} \pi c_{\mathrm{rw}}}
$$

for some $p$ : $0<p<1$, then there is $c_{t}\left(\gamma, \sigma^{2}, p\right)>0$ which is increasing in $t$ and satisfies

$$
\begin{aligned}
{ }^{\varepsilon} m_{t}^{1122}(\vec{x}) \leqslant & c_{t}\left(\gamma, \sigma^{2}, p\right)\left(\left\|\tilde{x}_{0}^{1}\right\|_{\infty} \vee\left\|\tilde{x}_{0}^{2}\right\|_{\infty}\right)^{4} \\
& \times\left(1+\int_{0}^{t} s^{-p}\left({ }^{\varepsilon} \mathrm{p}_{2 s}\left(x^{1}-x^{2}\right)+{ }^{\varepsilon} \mathrm{p}_{2 s}\left(x^{3}-x^{4}\right)\right) d s\right), \quad \forall t \geqslant 0,
\end{aligned}
$$

and

$$
\sup _{t \leqslant T} c_{t}\left(\gamma, \sigma^{2}, p\right)=c_{T}\left(\gamma, \sigma^{2}, p\right)<\infty, \quad \forall T>0 .
$$

In particular, if $p=1 / 2$, then

$$
\begin{aligned}
{ }^{\varepsilon} m_{t}^{1122}(\vec{x}) \leqslant & c_{t}\left(\gamma, \sigma^{2}\right)\left(\left\|\tilde{x}_{0}^{1}\right\|_{\infty} \vee\left\|\tilde{x}_{0}^{2}\right\|_{\infty}\right)^{4} \\
& \times\left(1+\int_{0}^{t} s^{-1 / 2}\left({ }^{\varepsilon} \mathrm{p}_{2 s}\left(x^{1}-x^{2}\right)+{ }^{\varepsilon} \mathrm{p}_{2 s}\left(x^{3}-x^{4}\right)\right) d s\right), \quad \forall t \geqslant 0 .
\end{aligned}
$$

These moment properties of $\widetilde{\mathbf{X}}$ play an important role in the proof of the dual propositions stated below.

Proposition 1.12 (Duality under (IntC)). - Assume $\gamma / \sigma^{2}<\left(\sqrt{6} \pi c_{\mathrm{rw}}\right)^{-1}$. Let $\left(\tilde{x}_{0}^{1}, \tilde{x}_{0}^{2}\right) \in\left(\mathcal{C}_{\text {rap }}^{+}\right)^{2}$ and $\widetilde{\mathbf{X}}$ be the particular solution of $(\mathbf{M P})_{\widetilde{\mathbf{X}}_{0}, \gamma}^{\sigma, \gamma}$ given by $(1.11)$ on some $\left(\widetilde{\Omega}, \widetilde{\mathcal{F}}, \widetilde{\mathcal{F}}_{t}, \widetilde{P}\right)$. Let $\mathbf{X}$ be any solution to $(\mathbf{M P})_{\mathbf{X}_{0}}^{\sigma, \gamma}$ satisfying $\left(\right.$ IntC) on $\left(\Omega, \mathcal{F}, \mathcal{F}_{t}, P\right)$ for some $\mathcal{F}_{0}$ measurable initial condition $\mathbf{X}_{0}$ satisfying $(\mathbf{E n C})$. Then for any $t \geqslant 0$ 


$$
\begin{aligned}
& P\left[\exp \left\{-\left\langle X_{t}^{1}+X_{t}^{2}, \tilde{x}_{0}^{1}+\tilde{x}_{0}^{2}\right\rangle+i\left\langle X_{t}^{1}-X_{t}^{2}, \tilde{x}_{0}^{1}-\tilde{x}_{0}^{2}\right\rangle\right\}\right] \\
& =\lim _{\varepsilon \downarrow 0} P \times \widetilde{P}\left[\operatorname { e x p } \left\{-\left\langle X_{0}^{1}+X_{0}^{2}, S_{\varepsilon}\left(\widetilde{X}_{t}^{1}+\widetilde{X}_{t}^{2}\right)\right\rangle\right.\right. \\
& \left.\left.\quad+i\left\langle X_{0}^{1}-X_{0}^{2}, S_{\varepsilon}\left(\widetilde{X}_{t}^{1}-\widetilde{X}_{t}^{2}\right)\right\rangle\right\}\right] .
\end{aligned}
$$

In particular, if $X_{0}^{j}(d x)=x_{0}^{j}(x) d x$ for some (deterministic) $x_{0}^{j} \in \mathcal{C}_{\text {tem }}^{+}$, then

$$
\begin{aligned}
P & {\left[\exp \left\{-\left\langle X_{t}^{1}+X_{t}^{2}, \tilde{x}_{0}^{1}+\tilde{x}_{0}^{2}\right\rangle+i\left\langle X_{t}^{1}-X_{t}^{2}, \tilde{x}_{0}^{1}-\tilde{x}_{0}^{2}\right\rangle\right\}\right] } \\
& =\widetilde{P}\left[\exp \left\{-\left\langle X_{0}^{1}+X_{0}^{2}, \widetilde{X}_{t}^{1}+\widetilde{X}_{t}^{2}\right\rangle+i\left\langle X_{0}^{1}-X_{0}^{2}, \widetilde{X}_{t}^{1}-\widetilde{X}_{t}^{2}\right\rangle\right\}\right] .
\end{aligned}
$$

Remark 1.13.-

(a) The restriction on $\gamma / \sigma^{2}$ is required for the existence of $\widetilde{\mathbf{X}}$ and (1.14). This restriction may be weakened to $\gamma \sigma^{-2}<2 / \sqrt{6}$ (see Remark 1.10).

(b) As $\widetilde{\mathbf{X}}$ is a fixed particular solution, the right hand side of $\left(D_{1}\right)$ depends only on $\mathcal{L}\left(\mathbf{X}_{0}\right)$. This will allow us to to derive the Markov property of solutions with finite initial conditions - see the proof of Theorem 1.9 below.

Proof of $\left(D_{1}\right) \rightarrow\left(D_{2}\right)$. - We will show that $\left(D_{2}\right)$ is an easy consequence of $\left(D_{1}\right)$. Note that

$$
S_{\varepsilon} x_{0}^{j}(x) \rightarrow x_{0}^{j}(x), \quad \forall x \in \mathbb{R}^{2},
$$

as $\varepsilon \downarrow 0$, and also by Lemma A.1 of the Appendix we have

$$
\sup _{\varepsilon \leqslant 1} S_{\varepsilon} x_{0}^{j}(x) \leqslant c_{A 1}(1, \lambda)\left|x_{0}^{j}\right|_{-\lambda} \phi_{-\lambda}(x), \quad j=1,2,
$$

for any $\lambda>0 . \widetilde{P}\left\langle\phi_{-\lambda}, \widetilde{\mathbf{X}}_{t}^{j}\right\rangle<\infty, j=1,2$, therefore by Dominated Convergence

$$
\left\langle X_{0}^{i}, S_{\varepsilon} \widetilde{X}_{t}^{j}\right\rangle=\left\langle S_{\varepsilon} x_{0}^{i}, \widetilde{X}_{t}^{j}\right\rangle \rightarrow\left\langle x_{0}^{i}, \widetilde{X}_{t}^{j}\right\rangle, \quad \widetilde{P} \text {-a.s. }, i, j=1,2 .
$$

A second application of Dominated Convergence allows us to take the limit in $\left(D_{1}\right)$ inside the $P \times \widetilde{P}$ and derive $\left(D_{2}\right)$.

We can get another version of Proposition 1.12 under finite initial conditions for larger class of test functions.

Proposition 1.14 (Duality under (IntC) for finite initial conditions). - Assume $\gamma / \sigma^{2}<\left(\sqrt{6} \pi c_{\mathrm{rw}}\right)^{-1}$. Let $\left(\tilde{x}_{0}^{1}, \tilde{x}_{0}^{2}\right) \in\left(\mathcal{C}_{\mathrm{b}}^{+}\right)^{2}$ and $\widetilde{\mathbf{X}}$ be the particular solution of (MP) $\widetilde{\mathbf{X}}_{0}^{\sigma, \gamma}$ given by (1.11) on some $\left(\widetilde{\Omega}, \widetilde{\mathcal{F}}, \widetilde{\mathcal{F}}_{t}, \widetilde{P}\right)$. Let $\mathbf{X}$ be any solution to $(\mathbf{M P})_{\mathbf{X}_{0}}^{\sigma, \gamma}$ satisfying (IntC) on $\left(\Omega, \mathcal{F}, \mathcal{F}_{t}, P\right)$ for some $\mathcal{F}_{0}$ measurable initial condition $\mathbf{X}_{0} \in \mathcal{M}_{\mathrm{f}, \mathrm{e}}$ satisfying (EnC). Then for any $t \geqslant 0\left(D_{1}\right)$ is satisfied, that is,

$$
\begin{aligned}
P & {\left[\exp \left\{-\left\langle X_{t}^{1}+X_{t}^{2}, \tilde{x}_{0}^{1}+\tilde{x}_{0}^{2}\right\rangle+i\left\langle X_{t}^{1}-X_{t}^{2}, \tilde{x}_{0}^{1}-\tilde{x}_{0}^{2}\right\rangle\right\}\right] } \\
& =\lim _{\varepsilon \downarrow 0} P \times \widetilde{P}\left[\exp \left\{-\left\langle X_{0}^{1}+X_{0}^{2}, S_{\varepsilon}\left(\widetilde{X}_{t}^{1}+\widetilde{X}_{t}^{2}\right)\right\rangle+i\left\langle X_{0}^{1}-X_{0}^{2}, S_{\varepsilon}\left(\widetilde{X}_{t}^{1}-\widetilde{X}_{t}^{2}\right)\right\rangle\right\}\right] .
\end{aligned}
$$


Organization of the paper. Section 2 is devoted to the proof of basic duality Proposition 1.12. The proof of Theorem 1.11(a) is completed in Corollary 2.11 of Section 2. In Section 3 we prove uniqueness Theorem 1.8 for general initial conditions. Theorem 1.11(b) follows as a trivial corollary of Theorem 1.8 and Theorem 1.11(a). Section 4 is devoted to continuity of transition function of mutually catalytic process with respect to initial conditions. In Section 5 we prove Theorem 1.9, Proposition 1.14 and show existence of the collision measure for a process satisfying (IntC).

The last section is Appendix A where some auxiliary results are presented and proved. Note that all the results in Appendix A are labeled with capital letter "A" instead of a section number (for example Lemma A.1).

\section{Proof of Proposition 1.12}

We start with proving of Proposition 1.12 under the stronger (SIntC) instead of (IntC). That is we are going to prove

Proposition 2.1 (Duality under (SIntC)). - Assume $\gamma / \sigma^{2}<\left(\sqrt{6} \pi c_{\mathrm{rw}}\right)^{-1}$. Let $\tilde{x}_{0}^{j} \in \mathcal{C}_{\text {rap }}^{+}$and $\widetilde{\mathbf{X}}$ be the particular solution of (MP) $\widetilde{\mathbf{X}}_{0}^{\sigma, \gamma}$ constructed in Section 1.4 on some $\left(\widetilde{\Omega}, \widetilde{\mathcal{F}}, \widetilde{\mathcal{F}}_{t}, \widetilde{P}\right)$. Let $\mathbf{X}$ be any solution to $(\mathbf{M P})_{\mathbf{X}_{0}}^{\sigma, \gamma}$ satisfying $(\mathbf{S I n t C})$ on $\left(\Omega, \mathcal{F}, \mathcal{F}_{t}, P\right)$ for some $\mathcal{F}_{0}$ measurable initial condition $\mathbf{X}_{0}$ satisfying $(\mathbf{E n C})$. Then for any $t \geqslant 0$

$$
\begin{aligned}
& P\left[\exp \left\{-\left\langle X_{t}^{1}+X_{t}^{2}, \tilde{x}_{0}^{1}+\tilde{x}_{0}^{2}\right\rangle+i\left\langle X_{t}^{1}-X_{t}^{2}, \tilde{x}_{0}^{1}-\tilde{x}_{0}^{2}\right\rangle\right\}\right] \\
& =\lim _{\varepsilon \downarrow 0} P \times \widetilde{P}\left[\operatorname { e x p } \left\{-\left\langle X_{0}^{1}+X_{0}^{2}, S_{\varepsilon}\left(\widetilde{X}_{t}^{1}+\widetilde{X}_{t}^{2}\right)\right\rangle\right.\right. \\
& \left.\left.\quad+i\left\langle X_{0}^{1}-X_{0}^{2}, S_{\varepsilon}\left(\widetilde{X}_{t}^{1}-\widetilde{X}_{t}^{2}\right)\right\rangle\right\}\right] .
\end{aligned}
$$

We start with some first and second moment results for solutions of (MP) $)_{\mathbf{X}_{0}}^{\sigma, \gamma}$ satisfying (SIntC) and (EnC). Throughout this section we assume $\mathbf{X}$ is such a solution with $\mathbf{X}_{0}$ possibly random. To simplify our notation in the following let

$$
\begin{gathered}
L_{t}(d x)=L(t, d x)=L_{\mathbf{X}}(t, d x), \\
L_{t}^{*, \varepsilon}(d x)=L^{*, \varepsilon}(t, d x)=L_{\mathbf{X}}^{*, \varepsilon}(t, d x), \\
L_{t}^{\varepsilon}(d x)=L^{\varepsilon}(t, d x)=L_{\mathbf{X}}^{\varepsilon}(t, d x) .
\end{gathered}
$$

The proof of the next lemma shows that a weakened form of (SIntC) (the $|x-y|^{-1}$ term may be dropped in the definition of $H_{\varepsilon, K}$ ) implies square integrability of $L_{t}(\varphi)$ for any $\varphi \in \mathcal{B}_{\mathrm{b} \text {,com. Nonetheless we continue to assume our stronger standing assumptions }}$ (SIntC) and (EnC).

LEMmA 2.2. - (a) For each $T>0, \varphi \in \mathcal{B}_{\mathrm{b} \text {,com }}$ there exists $c_{T, \varphi}<\infty$ such that

$$
\underset{\varepsilon \downarrow 0}{\limsup } P\left[L_{t}^{*, \varepsilon}(\varphi)^{2}+L_{t}^{\varepsilon}(\varphi)^{2}\right] \leqslant c_{T, \varphi} t, \quad \forall t \in[0, T] .
$$

In particular

$$
P\left[L_{t}(\varphi)^{2}\right] \leqslant c_{T, \phi} t, \quad \forall t \in[0, T], \forall \varphi \in \mathcal{B}_{\mathrm{b}, \mathrm{com}},
$$


and

$$
L_{t}^{*, \varepsilon}(\varphi) \stackrel{\mathrm{L}^{1}}{\rightarrow} L_{t}(\varphi)
$$

as $\varepsilon \downarrow 0$ for any $\varphi \in \mathcal{C}_{\text {com. }}$.

(b) If $\varphi_{1}, \varphi_{2} \in \mathcal{B}_{+}$and $T$ is a stopping time, then on $\{t \geqslant T\}$

$$
P\left[X_{t}^{j}\left(\varphi_{j}\right) \mid \mathcal{F}_{T}\right]=\left\langle X_{T}^{j}, S_{t-T} \varphi_{j}\right\rangle, \quad j=1,2,
$$

and

$$
P\left[X_{t}^{1}\left(\varphi_{1}\right) X_{t}^{2}\left(\varphi_{2}\right) \mid \mathcal{F}_{T}\right]=\left\langle X_{T}^{1}, S_{t-T} \varphi_{1}\right\rangle\left\langle X_{T}^{2}, S_{t-T} \varphi_{2}\right\rangle .
$$

(c) If $\varphi_{1}, \varphi_{2} \in \mathcal{B}_{+}$then

$$
P\left[X_{t}^{j}\left(\varphi_{j}\right)\right]=P\left[\left\langle X_{0}^{j}, S_{t} \varphi_{j}\right\rangle\right], \quad j=1,2, t>0
$$

and

$$
P\left[X_{t}^{1}\left(\varphi_{1}\right) X_{t}^{2}\left(\varphi_{2}\right)\right]=P\left[\left\langle X_{0}^{1}, S_{t} \varphi_{1}\right\rangle\left\langle X_{0}^{2}, S_{t} \varphi_{2}\right\rangle\right], \quad t>0
$$

where all these quantities are finite if $S_{t} \varphi_{j} \in \mathcal{B}_{\lambda}$ for some $\lambda>0$.

(d) If $\varphi: \mathbb{R}^{2} \mapsto[0, \infty]$ is Borel, then

$$
P\left[L_{t}(\varphi)\right]=\int_{0}^{t} \int_{\mathbb{R}^{2}} P\left[S_{s} X_{0}^{1}(x) S_{s} X_{0}^{2}(x)\right] \varphi(x) d x d s .
$$

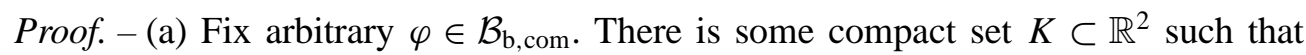
$\operatorname{supp}(\varphi) \subset K$. If $t \leqslant T$, then

$$
\begin{aligned}
P\left[L_{t}^{*, \varepsilon}(\varphi)^{2}\right] & =P\left[\left(\frac{1}{\varepsilon} \int_{0}^{\varepsilon} \int_{0}^{t} \int_{\mathbb{R}^{2}} S_{u} X_{s}^{1}(x) S_{u} X_{s}^{2}(x) \varphi(x) d x \frac{d s}{t} d u\right)^{2}\right] t^{2} \\
& \leqslant\|\varphi\|_{\infty}^{2} \frac{1}{\varepsilon} \int_{0}^{\varepsilon} d u \int_{0}^{t} \frac{d s}{t} P\left[\int_{K} S_{u} X_{s}^{1}(x) S_{u} X_{s}^{2}(x) d x^{2}\right] t^{2} \\
& \leqslant\|\varphi\|_{\infty}^{2} \frac{1}{\varepsilon} \int_{0}^{\varepsilon} d u \int_{0}^{t} d s P\left[H_{u, K}\left(\mathbf{X}_{s}\right)\right] t .
\end{aligned}
$$

An analogous bound holds for $P\left[L_{t}^{\varepsilon}(\varphi)^{2}\right]$ by an even simpler argument. (SIntC) now implies the first inequality in (a). Fatou's lemma gives the second. The above $L^{2}$ boundedness shows that the convergence in probability in the definition of $L_{t}(\varphi)$ may be strengthen to $L^{1}$ convergence.

(b) If $t \geqslant t_{0}>0$ are fixed we may argue as in Corollary 43 of [4] to see that if $\varphi \in \mathcal{C}_{\text {com }}^{\infty}$, then

$$
X_{t}^{j}(\varphi)=X_{t_{0}}^{j}\left(S_{t-t_{0}} \varphi\right)+\int_{0}^{t} \int_{\mathbb{R}^{2}} 1\left(r \geqslant t_{0}\right) S_{t-r} \varphi(x) d M^{(j)}(r, x), \quad j=1,2 .
$$


(See Chapter 2 of [16] for the construction and properties of the stochastic integral with respect to the orthogonal martingale measure $M^{(j)}$.) Let $t_{0}$ be a stopping time taking on finitely many values $\left\{t_{1}, \ldots, t_{n}\right\}$ with $\left\{t_{1}, \ldots, t_{n}\right\} \cap[0, t]=\left\{t_{1}, \ldots, t_{k}\right\}$. Then on $\left\{t_{0} \leqslant t\right\}$,

$$
\int_{0}^{t} \int_{\mathbb{R}^{2}} 1\left(r \geqslant t_{0}\right) S_{t-r} \varphi(x) d M^{(j)}(r, x)=\sum_{i=1}^{k} 1\left(t_{0}=t_{i}\right) \int_{t_{i}}^{t} \int_{\mathbb{R}^{2}} S_{t-r} \varphi(x) d M^{(j)}(r, x)
$$

as one can easily check by noting that $1\left(r \geqslant t_{0}\right) S_{t-r} \varphi(x)=1\left(r \geqslant t_{i}\right) S_{t-r} \varphi(x)$ on $\left\{t_{0}=t_{i}\right\}$ and then following the proof of the corresponding "localization" result for ordinary stochastic integrals (see Theorem 27 on p. 307 of Meyer [10]). It follows that (2.4) remains valid for the finite-valued stopping time $t_{0}$ on $\left\{t_{0} \leqslant t\right\}$. Let $T$ be an $\left(\mathcal{F}_{t}\right)$ stopping time and let $T_{n} \downarrow T$ be the standard set of finite-valued stopping times (we may allow $T_{n}=\infty$ ). Apply (2.4) with $T_{n}$ in place of $t_{0}$ and let $n \rightarrow \infty$ to see that on $\{T<t\} \subset \bigcup_{n=1}^{\infty}\left\{T_{n} \leqslant t\right\}$,

$$
X_{t}^{j}(\varphi)=X_{T}^{j}\left(S_{t-T} \varphi\right)+\int_{0}^{t} \int_{\mathbb{R}^{2}} 1(r \geqslant T) S_{t-r} \varphi(x) d M^{(j)}(r, x), \quad j=1,2 .
$$

This is of course trivial on $\{T=t\}$ (again using the localization result) and by taking bounded pointwise limits we see (2.5) is valid on $\{T \leqslant t\}$ for $\varphi \in \mathcal{B}_{\mathrm{b} \text {,com. }}$. We get by (a) and the definition of (MP) $\mathbf{X}_{0}^{\sigma, \gamma}$ that the stochastic integrals $\int_{0}^{s} \int_{\mathbb{R}^{2}} 1(r \geqslant$ T) $S_{t-r} \varphi(x) d M^{(j)}(r, x), s \leqslant t, j=1,2$, in (2.5) are orthogonal $L^{2}$ martingales and hence (b) follows for $\varphi_{j} \in \mathcal{B}_{\mathrm{b} \text {,com }}, j=1,2$. The boundedness and compact support conditions are readily dropped by Monotone Convergence.

(c) The equalities are immediate from (b) with $T=0$. The finiteness follows from (EnC) condition on $\mathbf{X}_{0}$.

(d) It follows from Monotone Convergence that, to establish the equality in (d), it suffices to consider non-negative bounded $\varphi$ with compact support. The $L^{1}$-convergence of $L_{t}^{*, \varepsilon}(\varphi)$ as $\varepsilon \downarrow 0$ from (a), and the second moment result in (c) imply

$$
\begin{aligned}
P\left[L_{t}(\varphi)\right] & =\lim _{\varepsilon \downarrow 0} P\left[L_{t}^{*, \varepsilon}(\varphi)\right] \\
& =\lim _{\varepsilon \downarrow 0} \int_{0}^{t} \int_{0}^{\varepsilon} \int_{\mathbb{R}^{2}} P\left[S_{u} X_{s}^{1}(x) S_{u} X_{s}^{2}(x)\right] \varphi(x) d x \frac{d u}{\varepsilon} d s \\
& =\lim _{\varepsilon \downarrow} \int_{0}^{t} \int_{0}^{\varepsilon} \int_{\mathbb{R}^{2}} P\left[S_{u+s} X_{0}^{1}(x) S_{u+s} X_{0}^{2}(x)\right] \varphi(x) d x \frac{d u}{\varepsilon} d s \\
& =\lim _{\varepsilon \downarrow 0} \int_{0}^{t} P\left[\int_{0}^{\varepsilon} \int_{\mathbb{R}^{2}} S_{u+s} X_{0}^{1}(x) S_{u+s} X_{0}^{2}(x) \varphi(x) d x \frac{d u}{\varepsilon}\right] d s .
\end{aligned}
$$

Let $G_{\varepsilon}(s)$ denotes the expression in square brackets. Then 


$$
\begin{aligned}
\int_{0}^{t} P & {\left[G_{\varepsilon}(s)^{2}\right] d s } \\
& \leqslant \int_{0}^{t} \int_{0}^{\varepsilon} P\left[\int_{\mathbb{R}^{2}} S_{u+s} X_{0}^{1}(x) S_{u+s} X_{0}^{2}(x) \varphi(x) d x^{2}\right] \frac{d u}{\varepsilon} d s \\
& =\int_{0}^{t} \int_{0}^{\varepsilon} P\left[P\left[\int_{\mathbb{R}^{2}} S_{u} X_{s}^{1}(x) S_{u} X_{s}^{2}(x) \varphi(x) d x \mid \mathcal{F}_{0}\right]^{2}\right] \frac{d u}{\varepsilon} d s \quad \text { (by (b) with } T=0 \text { ) } \\
& \leqslant \int_{0}^{t} \int_{0}^{\varepsilon} P\left[\int_{\mathbb{R}^{2}} S_{u} X_{s}^{1}(x) S_{u} X_{s}^{2}(x) \varphi(x) d x^{2}\right] \frac{d u}{\varepsilon} d s \quad \text { (by Jensen inequality) }
\end{aligned}
$$

which is bounded uniformly in $\varepsilon<\varepsilon_{0}$ by (SIntC). This allows us to take the limit through the first two integrals in (2.6) and conclude

$$
P\left[L_{t}(\varphi)\right]=\int_{0}^{t} P\left[\lim _{\varepsilon \downarrow 0} \int_{0}^{\varepsilon} \int_{\mathbb{R}^{2}} S_{u+s} X_{0}^{1}(x) S_{u+s} X_{0}^{2}(x) \varphi(x) d x \frac{d u}{\varepsilon}\right] d s<\infty
$$

We are implicitly assuming this limit exists. To this end we claim

$$
u \mapsto \int_{\mathbb{R}^{2}} S_{u} X_{0}^{1}(x) S_{u} X_{0}^{2}(x) \varphi(x) d x
$$

is continuous on $(0, \infty)$.

Note first that $u \mapsto S_{u} X_{0}^{j}(x)$ is continuous on $(0, \infty)$ by Corollary A.5. As $\varphi$ has compact support and, by Corollary A.4, $S_{u} X_{0}^{j}(x) \leqslant c_{A 3}(T, \lambda) c_{A 4}(\varepsilon) \phi_{-\lambda}(x) X_{0}^{j}\left(\phi_{\lambda}\right)$ for $\varepsilon \leqslant u \leqslant T$, (2.8) follows from application of Dominated Convergence. (2.8) shows that the limit in (2.7) is $\int_{\mathbb{R}^{2}} S_{s} X_{0}^{1}(x) S_{s} X_{0}^{2}(x) \varphi(x) d x$ for $s>0$ and this gives the equality in $(\mathrm{d})$.

In the following we assume that $\widetilde{\mathbf{X}}_{0} \in\left(\mathcal{C}_{\text {rap }}^{+}\right)^{2}$ and $\widetilde{\mathbf{X}}$ is a particular solution to $(\mathbf{M P}) \underset{\widetilde{\mathbf{X}}_{0}}{\sigma, \gamma}$ constructed in Section 1.4, which is independent of $\mathbf{X}$.

Denote by $\mathcal{C}_{T, \text { rap }}^{(1,2)}$ (respectively $\mathcal{C}_{T, \text { tem }}^{(1,2)}$ ) the set of all real-valued functions $\psi$ on $[0, T] \times \mathbb{R}^{2}$ such that $t \mapsto \psi(t, \cdot), t \mapsto \frac{\partial \psi(t, \cdot)}{\partial t}$ and $t \mapsto \Delta \psi(t, \cdot)$ are continuous $\mathcal{C}_{\text {rap }}$ (respectively $\mathcal{C}_{\text {tem }}$ ) valued functions.

LEMmA 2.3. - (a) Let $\left(\mu_{1}, \mu_{2}\right) \in \mathcal{M}_{\text {tem,e }}$ and $T>0$. Define $\mu=\mu_{1}+\mu_{2}+i\left(\mu_{2}-\right.$ $\left.\mu_{1}\right)$, and let $\bar{\mu}=\mu_{1}+\mu_{2}-i\left(\mu_{2}-\mu_{1}\right)$. Then

$$
\begin{aligned}
& \mathrm{e}^{-\left\langle\widetilde{X}_{t}^{1}, S_{T-t}(\mu)\right\rangle-\left\langle\widetilde{X}_{t}^{2}, S_{T-t}(\bar{\mu})\right\rangle}=\mathrm{e}^{-\left\langle\widetilde{X}_{0}^{1}, S_{T}(\mu)\right\rangle-\left\langle\widetilde{X}_{0}^{2}, S_{T}(\bar{\mu})\right\rangle} \\
& \quad+4 \gamma \int_{0}^{t} \mathrm{e}^{-\left\langle\widetilde{X}_{s}^{1}, S_{T-s}(\mu)\right\rangle-\left\langle\widetilde{X}_{s}^{2}, S_{T-s}(\bar{\mu})\right\rangle} S_{T-s}\left(\mu_{1}\right)(x) S_{T-s}\left(\mu_{2}\right)(x) L_{\widetilde{X}}(d s, d x)
\end{aligned}
$$




$$
\begin{aligned}
& -\int_{0}^{t} \int_{\mathbb{R}^{2}} \mathrm{e}^{-\left\langle\widetilde{X}_{s}^{1}, S_{T-s}(\mu)\right\rangle-\left\langle\widetilde{X}_{s}^{2}, S_{T-s}(\bar{\mu})\right\rangle} \\
& \times\left(S_{T-s}(\mu)(x) \widetilde{M}^{1}(d s, d x)+S_{T-s}(\bar{\mu})(x) \widetilde{M}^{2}(d s, d x)\right), \quad 0 \leqslant t<T,
\end{aligned}
$$

where $\widetilde{M}^{l}(d s, d x)(l=1,2)$ are martingale measures.

(b) Let $\left(\mu_{1}, \mu_{2}\right) \in \mathcal{M}_{\text {rap,e }}$ and $T>0$. Define $\mu=\mu_{1}+\mu_{2}+i\left(\mu_{2}-\mu_{1}\right)$, and let $\bar{\mu}=\mu_{1}+\mu_{2}-i\left(\mu_{2}-\mu_{1}\right)$. Then

$$
\begin{aligned}
& \mathrm{e}^{-\left\langle X_{t}^{1}, S_{T-t}(\mu)\right\rangle-\left\langle X_{t}^{2}, S_{T-t}(\bar{\mu})\right\rangle}=\mathrm{e}^{-\left\langle X_{0}^{1}, S_{T}(\mu)\right\rangle-\left\langle X_{0}^{2}, S_{T}(\bar{\mu})\right\rangle} \\
& \quad+4 \gamma \int_{0}^{t} \mathrm{e}^{-\left\langle X_{s}^{1}, S_{T-s}(\mu)\right\rangle-\left\langle X_{s}^{2}, S_{T-s}(\bar{\mu})\right\rangle} S_{T-s}\left(\mu_{1}\right)(x) S_{T-s}\left(\mu_{2}\right)(x) L_{\mathbf{X}}(d s, d x) \\
& \quad-\int_{0}^{t} \int_{\mathbb{R}^{2}} \mathrm{e}^{-\left\langle X_{s}^{1}, S_{T-s}(\mu)\right\rangle-\left\langle X_{s}^{2}, S_{T-s}(\bar{\mu})\right\rangle} \\
& \quad \times\left(S_{T-s}(\mu)(x) M^{1}(d s, d x)+S_{T-s}(\bar{\mu})(x) M^{2}(d s, d x)\right), \quad 0 \leqslant t<T,
\end{aligned}
$$

where $M^{l}(d s, d x)(l=1,2)$ are martingale measures.

Proof. - Arguing as in Lemma 42 of [4] we get

$$
\begin{aligned}
X_{t}^{j}\left(\psi_{t}\right) \equiv & X_{0}^{j}\left(\psi_{0}\right)+\int_{0}^{t} X_{s}^{j}\left(\frac{1}{2} \Delta \psi_{s}+\frac{\partial}{\partial s} \psi_{s}\right) d s \\
& +\int_{0}^{t} \int_{\mathbb{R}^{2}} \psi_{s}(x) M^{j}(d s, d x), \quad 0 \leqslant t<T, j=1,2, \psi \in \mathcal{C}_{T, \text { rap }}^{(1,2)} \\
\widetilde{X}_{t}^{j}\left(\psi_{t}\right) \equiv & \widetilde{X}_{0}^{j}\left(\psi_{0}\right)+\int_{0}^{t} \widetilde{X}_{s}^{j}\left(\frac{1}{2} \Delta \psi_{s}+\frac{\partial}{\partial s} \psi_{s}\right) d s \\
& +\int_{0}^{t} \int_{\mathbb{R}^{2}} \psi_{s}(x) \widetilde{M}^{j}(d s, d x), \quad 0 \leqslant t<T, j=1,2, \psi \in \mathcal{C}_{T, \text { tem }}^{(1,2)}
\end{aligned}
$$

(A little bit of care is needed to be able to take $\psi \in \mathcal{C}_{T \text {,tem }}^{(1,2)}$ in the second case-the proof uses Monotone Convergence and simple moment calculations.) By choosing functions $\psi_{t}^{1}=S_{T-t}(\mu), \psi_{t}^{2}=S_{T-t}(\bar{\mu})$ in (a), (b), and then applying Itô's formula on the interval $[0, T)$ one can readily complete the proof of the lemma.

LEMMA 2.4. - If $\widetilde{\mathbf{X}}_{0} \in\left(\mathcal{C}_{\text {rap }}^{+}\right)^{2}$ and $\widetilde{\mathbf{X}}$ is as above then $\widetilde{\mathbf{X}} . \in \Omega_{\text {rap }}$ a.s.

Proof. - By Theorem 11(c) of [3] we get that $\widetilde{\mathbf{X}} . \in \Omega_{0}$. To complete the proof we have to show that

$$
P\left[\sup _{t \leqslant T}\left\langle\widetilde{X}_{t}^{j}, \phi_{\lambda}\right\rangle\right]<\infty, \quad j=1,2, \forall T>0, \lambda<0
$$


First, for any $\lambda<0$, choose $\tilde{\varphi}_{\lambda} \in \mathcal{C}_{\text {tem }}^{(2)}$ such that $\tilde{\varphi}_{\lambda} \geqslant \phi_{\lambda}$. Then use (2.10) with $\psi_{t}(\cdot)=$ $\tilde{\varphi}_{\lambda}(\cdot)$ for all $t \geqslant 0$. The result follows easily by moment calculations and by Doob's inequality.

To simplify our notation (as in (2.1)-(2.3)) in the following let

$$
\begin{gathered}
\tilde{L}_{t}(d x)=\tilde{L}(t, d x)=L_{\widetilde{\mathbf{X}}}(t, d x), \\
\tilde{L}_{t}^{*, \varepsilon}(d x)=\tilde{L}^{*, \varepsilon}(t, d x)=L_{\widetilde{\mathbf{x}}}^{*, \varepsilon}(t, d x), \\
\tilde{L}_{t}^{\varepsilon}(d x)=\tilde{L}^{\varepsilon}(t, d x)=L_{\widetilde{\mathbf{x}}}^{\varepsilon}(t, d x) .
\end{gathered}
$$

LEMMA 2.5. - Let $T$ be any bounded stopping time and $\varphi$ be a random function such that

$$
\varphi \in L^{1}\left(\mathbb{R}^{2} \times \Omega, L_{t}(\omega, d x) P(d \omega)\right), \quad \forall t>0
$$

and $\varphi$ is $\mathcal{B} \times \mathcal{F}_{T}$-measurable. Then

$$
P\left[L([T, T+t] \times \varphi) \mid \mathcal{F}_{T}\right]=\int_{T}^{T+t} \int_{\mathbb{R}^{2}} S_{u-T}\left(X_{T}^{1}\right)(x) S_{u-T}\left(X_{T}^{2}\right)(x) \varphi(x) d x d u, \quad P \text {-a.s. }
$$

Proof. - Take a non-random $\varphi \in \mathcal{C}_{\text {com. }} L_{t}$ is a continuous measure-valued process, therefore $L_{t}(\varphi)$ is continuous. If $T$ is a bounded stopping time, then by Dominated Convergence and Lemma 2.2(a)

$$
\lim _{\delta \downarrow 0} P\left[L_{T+\delta}(\varphi)-L_{T}(\varphi) \mid \mathcal{F}_{T}\right]=0, \quad P \text {-a.s. }
$$

Hence, for any $\delta>0$, for $P$-a.e $\omega$ there exists $\delta^{\prime}(\omega)>0, \delta^{\prime} \in \mathcal{F}_{T}$ such that

$$
P\left[L\left(\left[T, T+\delta^{\prime \prime}\right] \times|\varphi|\right) \mid \mathcal{F}_{T}\right] \leqslant \delta, \quad \forall 0 \leqslant \delta^{\prime \prime} \leqslant \delta^{\prime} .
$$

Arguing as in the proof of Lemma 2.2(b), that is by using approximation of the stopping time $T$ with finite values stopping times and continuity of $L_{t}$ we can show that

$$
P\left[L_{T+t}(\varphi)-L_{T+\delta^{\prime \prime}}(\varphi) \mid \mathcal{F}_{T}\right]=P\left[\lim _{\varepsilon \downarrow 0} L_{T+t}^{*, \varepsilon}(\varphi)-L_{T+\delta^{\prime \prime}}^{*, \varepsilon}(\varphi) \mid \mathcal{F}_{T}\right] .
$$

By Lemma 2.2(a) $L^{*, \varepsilon}$ converges to $L$ in $L^{1}$, therefore

$$
\begin{aligned}
P & {\left[L_{T+t}(\varphi)-L_{T+\delta^{\prime \prime}}(\varphi) \mid \mathcal{F}_{T}\right] } \\
& =\lim _{\varepsilon \downarrow 0} P\left[L_{T+t}^{*, \varepsilon}(\varphi)-L_{T+\delta^{\prime \prime}}^{*, \varepsilon}(\varphi) \mid \mathcal{F}_{T}\right] \\
& =\lim _{\varepsilon \downarrow 0} \int_{T+\delta^{\prime \prime}}^{T+t} \int_{\mathbb{R}^{2}} \frac{1}{\varepsilon} \int_{0}^{\varepsilon} P\left[S_{u}\left(X_{s}^{1}\right)(x) S_{u}\left(X_{s}^{2}\right)(x) \mid \mathcal{F}_{T}\right] \varphi(x) d u d x d s \\
& =\lim _{\varepsilon \downarrow 0} \int_{T+\delta^{\prime \prime}}^{T+t} \int_{\mathbb{R}^{2}} \frac{1}{\varepsilon} \int_{0}^{\varepsilon} S_{u+s-T}\left(X_{T}^{1}\right)(x) S_{u+s-T}\left(X_{T}^{2}\right)(x) \varphi(x) d u d x d s
\end{aligned}
$$




$$
=\int_{T+\delta^{\prime \prime}}^{T+t} \int_{\mathbb{R}^{2}} S_{s-T}\left(X_{T}^{1}\right)(x) S_{s-T}\left(X_{T}^{2}\right)(x) \varphi(x) d x d s, \quad \forall 0 \leqslant \delta^{\prime \prime} \leqslant \delta^{\prime},
$$

where the second equality is a consequence of Fubini's theorem and the third equality follows by Lemma 2.2(b). Hence, we get that

$$
\begin{aligned}
& \left|P\left[L([T, T+t] \times \varphi) \mid \mathcal{F}_{T}\right]-\int_{T+\delta^{\prime \prime}}^{T+t} \int_{\mathbb{R}^{2}} S_{u-T}\left(X_{T}^{1}\right)(x) S_{u-T}\left(X_{T}^{2}\right)(x) \varphi(x) d x d u\right| \leqslant \delta, \\
& \quad \forall 0<\delta^{\prime \prime} \leqslant \delta^{\prime}, P \text {-a.s. }
\end{aligned}
$$

Letting $\delta^{\prime \prime} \downarrow 0$, (2.14) follows since $\delta$ was arbitrary and $L_{t}$ is continuous.

The extension of (2.14) for any $\mathcal{B} \times \mathcal{F}_{T}$-measurable

$$
\varphi \in L^{1}\left(\mathbb{R}^{2} \times \Omega, L_{t}(\omega, d x) P(d \omega)\right), \quad \forall t>0,
$$

is trivial.

The next lemma does not give $\lim _{\varepsilon \downarrow 0} L^{\varepsilon}=L$ but it is nonetheless quite useful.

LEMMA 2.6. - Let $g$ be Borel $\times$ optional-measurable function on $\mathbb{R}_{+} \times \mathbb{R}^{2} \times \Omega$ such that for $P$-a.e. $\omega$ the mapping

$$
s \mapsto g(s, \cdot, \omega)
$$

is continuous from $\mathbb{R}_{+}$to $\mathcal{C}$ and for each compact set $K \subset \mathbb{R}^{2}$ and $t>0$, there exists a constant $C_{K, t}$ such that

$$
\|g\|_{t, K} \equiv \sup _{0 \leqslant s \leqslant t, x \in K}|g(s, x, \omega)| \leqslant C_{K, t}, \quad P \text {-a.s. }
$$

Assume also that

$$
\begin{gathered}
\sup _{0<\varepsilon \leqslant 1} P\left[\int_{0}^{t} \int_{\mathbb{R}^{2}}|g(s, x, \cdot)| L^{\varepsilon}(d s, d x)\right]+P\left[\int_{0}^{t} \int_{\mathbb{R}^{2}}|g(s, x, \cdot)| L(d s, d x)\right]<\infty, \\
\lim _{k \rightarrow \infty} \sup _{0<\varepsilon \leqslant 1} P\left[\int_{0}^{t} \int_{|x|>k}|g(s, x, \cdot)| L^{\varepsilon}(d s, d x)\right]=0 .
\end{gathered}
$$

Then

$$
\lim _{\varepsilon \downarrow 0} P\left[\int_{0}^{t} \int_{\mathbb{R}^{2}} g(s, x, \cdot) L^{\varepsilon}(d s, d x)\right]=P\left[\int_{0}^{t} \int_{\mathbb{R}^{2}} g(s, x, \cdot) L(d s, d x)\right] .
$$

Proof. - For any $\delta^{\prime}>0$ choose a compact set $K_{\delta^{\prime}} \subset \mathbb{R}^{2}$ such that

$$
\sup _{0<\varepsilon \leqslant 1} P\left[\int_{0}^{t} \int_{K_{\delta^{\prime}}^{c}}|g(s, x, \cdot)| L^{\varepsilon}(d s, d x)\right]+P\left[\int_{0}^{t} \int_{K_{\delta^{\prime}}^{c}}|g(s, x, \cdot)| L(d s, d x)\right] \leqslant \delta^{\prime} / 3 .
$$


By Lemma 2.5 for any $t \geqslant 0$ and $\delta>0$ and any $\mathcal{B} \times \mathcal{F}_{t}$-measurable, $L_{t+\delta}(\omega, d x) P(d \omega)$ integrable function $\varphi(\omega, x)$, we have

$$
P\left[L\left(\varphi 1_{K_{\delta^{\prime}}} \times[t, t+\delta]\right) \mid \mathcal{F}_{t}\right]=\int_{t}^{t+\delta} \int_{K_{\delta^{\prime}}} S_{u-t}\left(X_{t}^{1}\right)(x) S_{u-t}\left(X_{t}^{2}\right)(x) \varphi(x) d x d u .
$$

This means that for any optional step function $f=\sum_{k=1}^{n} \varphi_{k} 1\left(\left[t_{k}, t_{k+1}\right)\right)$, where $\varphi_{k}$ is an $\mathcal{B} \times \mathcal{F}_{t_{k}}$-measurable $L_{t}(\omega, d x) P(d \omega)$-integrable function, we have

$$
\begin{aligned}
\lim _{\varepsilon \downarrow 0} P & {\left[\int_{0}^{t} \int_{K_{\delta^{\prime}}} f(s, x, \cdot) L^{\varepsilon}(d s, d x)\right] } \\
= & \lim _{\varepsilon \downarrow 0} P\left[\sum_{k=1}^{n} \int_{t_{k} \wedge t}^{t_{k+1} \wedge t} \int_{K_{\delta^{\prime}}} S_{\varepsilon+u-t_{k}}\left(X_{t_{k}}^{1}\right)(x)\right. \\
& \left.\times S_{\varepsilon+u-t_{k}}\left(X_{t_{k}}^{2}\right)(x) \varphi_{k}(x) d x d u\right] \quad \text { (by Lemma 2.2(b)) } \\
= & P\left[\int_{0}^{t} \int_{K_{\delta^{\prime}}} f(s, x, \cdot) L(d s, d x)\right] \quad \text { (by Lemma 2.5). }
\end{aligned}
$$

For arbitrary $g$ (indicated in the assumptions of the theorem) the procedure is standard. Define:

$$
\begin{aligned}
& t_{0}^{k}=0, \\
& t_{n+1}^{k}=t_{n}^{k}+2^{-k}, \\
& g^{k}(t)=g\left(t_{n}^{k}\right), \quad t_{n}^{k} \leqslant t<t_{n+1}^{k}, \\
& A_{k, \delta^{\prime \prime}}=\left\{\omega: \sup _{0 \leqslant s \leqslant t, x \in K_{\delta^{\prime}}}\left|g(s, x, \omega)-g^{k}(s, x, \omega)\right|<\delta^{\prime \prime}\right\}
\end{aligned}
$$

$g(\cdot, \cdot, \omega)$ is continuous $P$-a.s. and so for each $0<\delta, \delta^{\prime \prime}<1$ there exists $K>0$ such that

$$
\begin{gathered}
P\left(A_{k, \delta^{\prime \prime}}\right)>1-\delta, \quad \forall k \geqslant K . \\
P\left[\int_{0}^{t} \int_{K_{\delta^{\prime}}} g(s, x, \cdot) L^{\varepsilon}(d s, d x)\right]=P\left[\int_{0}^{t} \int_{K_{\delta^{\prime}}} g(s, x, \cdot)-g^{k}(s, x, \cdot) L^{\varepsilon}(d s, d x)\right] \\
+P\left[\int_{0}^{t} \int_{K_{\delta^{\prime}}} g^{k}(s, x, \cdot) L^{\varepsilon}(d s, d x)\right], \quad \forall k \geqslant 1 .
\end{gathered}
$$

$P\left[L_{t}^{\varepsilon}\left(K_{\delta^{\prime}}\right)\right], P\left[L_{t}^{\varepsilon}\left(K_{\delta^{\prime}}\right)^{2}\right], P\left[L_{t}\left(K_{\delta^{\prime}}\right)\right], P\left[L_{t}\left(K_{\delta^{\prime}}\right)^{2}\right]$ are bounded by Lemma 2.2(a). Choose $\delta^{\prime \prime}$ such that

$$
\delta^{\prime \prime}\left(P\left[L_{t}^{\varepsilon}\left(K_{\delta^{\prime}}\right)\right]+P\left[L_{t}\left(K_{\delta^{\prime}}\right)\right]\right) \leqslant \delta^{\prime} / 3
$$


and choose $\delta$ such that

$$
2\|g\|_{t, K_{\delta^{\prime}}}\left(\sqrt{P\left[L_{t}^{\varepsilon}\left(K_{\delta^{\prime}}\right)^{2}\right] \delta}+\sqrt{P\left[L_{t}\left(K_{\delta^{\prime}}\right)^{2}\right] \delta}\right) \leqslant \delta^{\prime} / 3 .
$$

Now take $k$ such that (2.17) is satisfied. Then

$$
\begin{aligned}
& P\left[\int_{0}^{t} \int_{K_{\delta^{\prime}}}\left|g(s, x, \cdot)-g^{k}(s, x, \cdot)\right|\left(L^{\varepsilon}(d s, d x)+L(d s, d x)\right)\right] \\
& \leqslant P\left[1_{A_{k, \delta^{\prime \prime}}}(\cdot) \int_{0}^{t} \int_{K_{\delta^{\prime}}}\left|g(s, x, \cdot)-g^{k}(s, x, \cdot)\right|\left(L^{\varepsilon}(d s, d x)+L(d s, d x)\right)\right] \\
& +P\left[1_{A_{k, \delta^{\prime \prime}}^{c}}(\cdot) \int_{0}^{t} \int_{K_{\delta^{\prime}}}\left|g(s, x, \cdot)-g^{k}(s, x, \cdot)\right|\left(L^{\varepsilon}(d s, d x)+L(d s, d x)\right)\right] \\
& \leqslant \delta^{\prime \prime} P\left[1_{A_{k, \delta^{\prime \prime}}}(\cdot)\left(L_{t}^{\varepsilon}\left(K_{\delta^{\prime}}\right)+L_{t}\left(K_{\delta^{\prime}}\right)\right)\right] \\
& +2\|g\|_{t, K_{\delta^{\prime}}} P\left[1_{A_{k, \delta^{\prime \prime}}^{c}}(\cdot)\left(L_{t}^{\varepsilon}\left(K_{\delta^{\prime}}\right)+L_{t}\left(K_{\delta^{\prime}}\right)\right)\right] \\
& \leqslant \delta^{\prime \prime}\left(P\left[L_{t}^{\varepsilon}\left(K_{\delta^{\prime}}\right)\right]+P\left[L_{t}\left(K_{\delta^{\prime}}\right)\right]\right) \\
& +2\|g\|_{t, K_{\delta^{\prime}}}\left(\sqrt{P\left[L_{t}^{\varepsilon}\left(K_{\delta^{\prime}}\right)^{2}\right] P\left(A_{k, \delta^{\prime \prime}}^{c}\right)}+\sqrt{P\left[L_{t}\left(K_{\delta^{\prime}}\right)^{2}\right] P\left(A_{k, \delta^{\prime \prime}}^{c}\right)}\right) \\
& \leqslant \frac{2 \delta^{\prime}}{3}, \quad \forall 0<\varepsilon \leqslant 1 \text {. }
\end{aligned}
$$

Letting $\varepsilon \downarrow 0$ in (2.18) we get by (2.15), (2.16), (2.19) that

$$
\begin{aligned}
& \underset{\varepsilon \downarrow 0}{\limsup }\left|P\left[\int_{0}^{t} \int_{\mathbb{R}^{2}} g(s, x, \cdot) L^{\varepsilon}(d s, d x)\right]-P\left[\int_{0}^{t} \int_{\mathbb{R}^{2}} g(s, x, \cdot) L(d s, d x)\right]\right| \\
& \quad \leqslant \delta^{\prime} / 3+2 \delta^{\prime} / 3=\delta^{\prime} .
\end{aligned}
$$

Since $\delta^{\prime}$ was arbitrary we are done.

To simplify our notation in the following let

$$
\begin{aligned}
& \mathfrak{E}(\mu, \tilde{\mu}) \equiv \exp \left\{-\left\langle\mu_{1}+\mu_{2}, \tilde{\mu}_{1}+\tilde{\mu}_{2}\right\rangle+i\left\langle\mu_{1}-\mu_{2}, \tilde{\mu}_{1}-\tilde{\mu}_{2}\right\rangle\right\}, \\
& \quad \text { for } \mu=\left(\mu_{1}, \mu_{2}\right) \in \mathcal{M} \times \mathcal{M}, \tilde{\mu}=\left(\tilde{\mu}_{1}, \tilde{\mu}_{2}\right) \in \mathcal{B}_{+} \times \mathcal{B}_{+} \\
& \quad \text { or } \mu=\left(\mu_{1}, \mu_{2}\right) \in \mathcal{B}_{+} \times \mathcal{B}_{+}, \tilde{\mu}=\left(\tilde{\mu}_{1}, \tilde{\mu}_{2}\right) \in \mathcal{M} \times \mathcal{M}, \\
& L^{t}([0, s] \times B) \equiv L([0, t] \times B)-L([0, t-s] \times B), \quad 0 \leqslant s \leqslant t, \\
& L^{t, \varepsilon}([0, s] \times B) \equiv L^{\varepsilon}([0, t] \times B)-L^{\varepsilon}([0, t-s] \times B), \quad 0 \leqslant s \leqslant t,
\end{aligned}
$$

and we set $\mathfrak{E}(\mu, \tilde{\mu})=0$ if $\left\langle\mu_{1}+\mu_{2}, \tilde{\mu}_{1}+\tilde{\mu}_{2}\right\rangle=\infty$. For $\mu=\left(\mu_{1}, \mu_{2}\right) \in \mathcal{M} \times \mathcal{M}$ we set $S_{t} \mu \equiv\left(S_{t} \mu_{1}, S_{t} \mu_{2}\right)$. Given the Polish space $E$ and the space of Radon measures $\mathcal{M}(E)$ 
on $E$, let $P$ be any probability measure on $\mathcal{M}(E)$. If the measure $\hat{\mu} \in \mathcal{M}(E)$ defined by $\hat{\mu}(A)=\int_{\mathcal{M}(E)} \mu(A) P(d \mu)$ has a density, this density, with a slight abuse of notation, will be denoted by $P[\mu(x)]$. For example we will write

$$
\begin{array}{r}
\widetilde{P}\left[\int_{0}^{t} \int_{\mathbb{R}^{2}} \mathfrak{E}\left(\mathbf{X}_{t-s}, S_{\varepsilon}\left(\widetilde{\mathbf{X}}_{s}\right)\right) \psi(s, x) \tilde{L}(d s, d x)\right] \\
=\int_{0}^{t} \int_{\mathbb{R}^{2}} \tilde{P}\left[\mathfrak{E}\left(\mathbf{X}_{t-s}, S_{\varepsilon}\left(\widetilde{\mathbf{X}}_{s}\right)\right) \psi(s, x) \tilde{L}(s, x)\right] d s d x, \\
P\left[\int_{0}^{t} \int_{\mathbb{R}^{2}} \mathfrak{E}\left(\mathbf{X}_{s}, S_{\varepsilon}\left(\widetilde{\mathbf{X}}_{t-s}\right)\right) \psi(s, x) L(d s, d x)\right] \\
\quad=\int_{0}^{t} \int_{\mathbb{R}^{2}} P\left[\mathfrak{E}\left(\mathbf{X}_{s}, S_{\varepsilon}\left(\widetilde{\mathbf{X}}_{t-s}\right)\right) \psi(s, x) L(s, x)\right] d s d x,
\end{array}
$$

for any integrable function $\psi$ on $\mathbb{R}_{+} \times \mathbb{R}^{2}$. Recall that the above measures are absolutely continuous (and therefore densities are well-defined) since $\widetilde{P}[\tilde{L}(d s, d x)]$ and $P[L(d s, d x)]$ are absolutely continuous by Lemma 2.2(d) (note that $\widetilde{X}$ also satisfies the hypotheses of Lemma 2.2).

LEMMA 2.7. - For any $t, \varepsilon>0$

$$
\begin{aligned}
P \times & \widetilde{P}\left[\mathfrak{E}\left(\mathbf{X}_{t}, S_{\varepsilon}\left(\widetilde{\mathbf{X}}_{0}\right)\right)\right]-P \times \widetilde{P}\left[\mathfrak{E}\left(\mathbf{X}_{0}, S_{\varepsilon}\left(\widetilde{\mathbf{X}}_{t}\right)\right)\right] \\
= & 4 \gamma P\left[\int_{0}^{t} \int_{\mathbb{R}^{2}} \widetilde{P}\left[\mathfrak{E}\left(\mathbf{X}_{t-s}, S_{\varepsilon}\left(\widetilde{\mathbf{X}}_{s}\right)\right)\left(S_{\varepsilon} \widetilde{X}_{s}^{1}\right)(x)\left(S_{\varepsilon} \widetilde{X}_{s}^{2}\right)(x)\right] L^{t}(d s, d x)\right. \\
& \left.-\int_{0}^{t} \int_{\mathbb{R}^{2}} \widetilde{P}\left[\mathfrak{E}\left(\mathbf{X}_{t-s}, S_{\varepsilon}\left(\widetilde{\mathbf{X}}_{s}\right)\right) \tilde{L}(s, x)\right] L^{t, \varepsilon}(d s, d x)\right] .
\end{aligned}
$$

Proof. - Fix any $T>0$ and define three functions: $h_{1}, h_{2}, f$ by

$$
\begin{gathered}
f(t, s)=P \times \widetilde{P}\left[\mathfrak{E}\left(\mathbf{X}_{t}, S_{T-t-s}\left(\widetilde{\mathbf{X}}_{s}\right)\right)\right], \\
h_{1}(t, s)=\int_{\mathbb{R}^{2}} 4 \gamma \widetilde{P} \times P\left[\mathfrak{E}\left(\mathbf{X}_{t}, S_{T-t-s}\left(\widetilde{\mathbf{X}}_{s}\right)\right)\left(S_{T-t-s} \widetilde{X}_{s}^{1}\right)(x)\left(S_{T-t-s} \widetilde{X}_{s}^{2}\right)(x) L(t, x)\right] d x, \\
h_{2}(t, s)=\int_{\mathbb{R}^{2}} 4 \gamma \widetilde{P} \times P\left[\mathfrak{E}\left(\mathbf{X}_{t}, S_{T-t-s}\left(\widetilde{\mathbf{X}}_{s}\right)\right)\left(S_{T-t-s} X_{t}^{1}\right)(x)\left(S_{T-t-s} X_{t}^{2}\right)(x) \tilde{L}(s, x)\right] d x
\end{gathered}
$$

for $0 \leqslant s+t<T$.

By Lemma 2.3 (it is easy to check that stochastic integrals with respect to martingale measures are in fact martingales due to $L^{2}$ boundedness) and Fubini's theorem we have

$$
f(t, s)=f(0, s)+\int_{0}^{t} h_{1}(u, s) d u, \quad \forall t, s \geqslant 0: t+s<T,
$$




$$
f(t, s)=f(t, 0)+\int_{0}^{s} h_{2}(t, u) d u, \quad \forall t, s \geqslant 0: t+s<T .
$$

From Lemma 4.4 .10 of [7] (see e.g. Lemma 4.17 [11]) it follows that

$$
f(t, 0)-f(0, t)=\int_{0}^{t} h_{1}(t-s, s)-h_{2}(t-s, s) d s
$$

for almost every $t, 0 \leqslant t<T$. To verify (2.20) for every $0 \leqslant t<T$ it is enough to show the continuity of the right side of (2.20). Take an arbitrary $t_{n} \rightarrow t$ and check that

$$
\int_{0}^{T} 1\left(s \leqslant t_{n}\right) h_{i}\left(t_{n}-s, s\right) d s \rightarrow \int_{0}^{T} 1(s \leqslant t) h_{i}(t-s, s) d s, \quad i=1,2 .
$$

Consider (2.21) for $i=1$. To simplify the notation define

$$
\hat{f}\left(t_{n}, s\right)=\mathfrak{E}\left(\mathbf{X}_{s}, S_{T-t_{n}}\left(\tilde{\mathbf{X}}_{t_{n}-s}\right)\right) .
$$

Then

$$
\begin{gathered}
\int_{0}^{T} 1\left(s \leqslant t_{n}\right) h_{1}\left(t_{n}-s, s\right) d s=\int_{0}^{T} 1\left(s \leqslant t_{n}\right) h_{1}\left(s, t_{n}-s\right) d s \\
=4 \gamma \widetilde{P} \times P\left[\int_{0}^{T} \int_{\mathbb{R}^{2}} 1\left(s \leqslant t_{n}\right) \hat{f}\left(t_{n}, s\right)\right. \\
\left.\quad \times\left(S_{T-t_{n}} \widetilde{X}_{t_{n}-s}^{1}\right)(x)\left(S_{T-t_{n}} \tilde{X}_{t_{n}-s}^{2}\right)(x) L(d s, d x)\right] .
\end{gathered}
$$

By Lemma $2.4 \widetilde{X}^{1}, \widetilde{X}^{2}$ are continuous in $\mathcal{M}_{\text {rap }}$. This together with Corollaries A.4, A.5 and Dominated Convergence implies that

$$
\begin{aligned}
1(s & \left.\leqslant t_{n}\right) \hat{f}\left(t_{n}, s\right)\left(S_{T-t_{n}} \tilde{X}_{t_{n}-s}^{1}\right)(x)\left(S_{T-t_{n}} \tilde{X}_{t_{n}-s}^{2}\right)(x) \\
& \rightarrow \hat{f}(t, s)\left(S_{T-t} \widetilde{X}_{t-s}^{1}\right)(x)\left(S_{T-t} \widetilde{X}_{t-s}^{2}\right)(x), \quad \text { as } n \rightarrow \infty
\end{aligned}
$$

for every $x \in \mathbb{R}^{2}, s<t, P \times \widetilde{P}$-a.s. Note that

$$
\begin{aligned}
& \lim _{t_{n} \rightarrow t} \widetilde{P} \times P\left[\int_{0}^{T} \int_{\mathbb{R}^{2}}^{T} 1\left(s \leqslant t_{n}\right)\left(S_{T-t_{n}} \widetilde{X}_{t_{n}-s}^{1}\right)(x)\left(S_{T-t_{n}} \widetilde{X}_{t_{n}-s}^{2}\right)(x) L(d s, d x)\right] \\
& \quad=\lim _{t_{n} \rightarrow t} \int_{0}^{t_{n}} \int_{\mathbb{R}^{2}} \widetilde{P} \times P\left[\left(S_{T-s} \widetilde{X}_{0}^{1}\right)(x)\left(S_{T-s} \widetilde{X}_{0}^{2}\right)(x)\left(S_{s} X_{0}^{1}\right)(x)\left(S_{s} X_{0}^{2}\right)(x)\right] d x d s
\end{aligned}
$$




$$
\begin{aligned}
& =\int_{0}^{t} \int_{\mathbb{R}^{2}} \widetilde{P} \times P\left[\left(S_{T-s} \widetilde{X}_{0}^{1}\right)(x)\left(S_{T-s} \widetilde{X}_{0}^{2}\right)(x)\left(S_{s} X_{0}^{1}\right)(x)\left(S_{s} X_{0}^{2}\right)(x)\right] d x d s \\
& =\widetilde{P} \times P\left[\int_{0}^{T} \int_{\mathbb{R}^{2}} 1(s \leqslant t)\left(S_{T-t} \widetilde{X}_{t-s}^{1}\right)(x)\left(S_{T-t} \widetilde{X}_{t-s}^{2}\right)(x) L(d s, d x)\right] .
\end{aligned}
$$

Define $\mu(d s, d x, d \tilde{\omega}, d \omega) \equiv L(\omega, d s, d x) \widetilde{P}(d \tilde{\omega}) P(d \omega)$. Then (2.22), (2.23) imply that convergence

$$
\begin{aligned}
& 1\left(s \leqslant t_{n}\right)\left(S_{T-t_{n}} \widetilde{X}_{t_{n}-s}^{1}\right)(x)\left(S_{T-t_{n}} \widetilde{X}_{t_{n}-s}^{2}\right)(x) \\
& \quad \rightarrow 1(s \leqslant t)\left(S_{T-t} \widetilde{X}_{t-s}^{1}\right)(x)\left(S_{T-t} \widetilde{X}_{t-s}^{2}\right)(x), \quad \text { as } n \rightarrow \infty,
\end{aligned}
$$

is in $L^{1}(\mu(d s, d x, d \tilde{\omega}, d \omega))$. Since $\hat{f}\left(t_{n}, s\right)$ is uniformly bounded in $n$ we get that

$$
\begin{aligned}
& 1\left(s \leqslant t_{n}\right) \hat{f}\left(t_{n}, s\right)\left(S_{T-t_{n}} \widetilde{X}_{t_{n}-s}^{1}\right)(x)\left(S_{T-t_{n}} \widetilde{X}_{t_{n}-s}^{2}\right)(x) \\
& \quad \rightarrow 1(s \leqslant t) \hat{f}(t, s)\left(S_{T-t} \widetilde{X}_{t-s}^{1}\right)(x)\left(S_{T-t} \widetilde{X}_{t-s}^{2}\right)(x)
\end{aligned}
$$

in $L^{1}(\mu(d s, d x, d \tilde{\omega}, d \omega))$ and (2.21) follows for $i=1$. By the same argument it is easy to show that (2.21) holds for $i=2$ and the continuity of the right hand side of (2.20) follows. Hence (2.20) is satisfied for each $0<t<T$. Take $T=t+\varepsilon$ and the proof is complete.

Fix $t>0$. To simplify the notation denote

$$
\begin{gathered}
\varphi_{s}^{\varepsilon} \equiv \mathfrak{E}\left(\mathbf{X}_{s}, S_{\varepsilon}\left(\widetilde{\mathbf{X}}_{t-s}\right)\right) \\
f^{\varepsilon, \varepsilon^{\prime}}(s, x) \equiv\left(S_{\varepsilon} \widetilde{X}_{t-s}^{1}(x) S_{\varepsilon} \widetilde{X}_{t-s}^{2}(x)\right)\left(S_{\varepsilon^{\prime}} X_{s}^{1}(x) S_{\varepsilon^{\prime}} X_{s}^{2}(x)\right) .
\end{gathered}
$$

Use Lemma 2.7 and the equality in (2.24) with $s=t$ to see

$$
\begin{aligned}
I_{\varepsilon} \equiv & \left|P \times \widetilde{P}\left[\varphi_{t}^{\varepsilon}-\varphi_{0}^{\varepsilon}\right]\right| \\
= & \mid 4 \gamma P\left[\int_{0}^{t} \int_{\mathbb{R}^{2}} \widetilde{P}\left[\varphi_{s}^{\varepsilon} S_{\varepsilon} \widetilde{X}_{t-s}^{1}(x) S_{\varepsilon} \widetilde{X}_{t-s}^{2}(x)\right] L(d s, d x)\right. \\
& \left.-\int_{0}^{t} \int_{\mathbb{R}^{2}} \widetilde{P}\left[\varphi_{t-s}^{\varepsilon} \tilde{L}(s, x) S_{\varepsilon} X_{t-s}^{1}(x) S_{\varepsilon} X_{t-s}^{2}(x)\right] d x d s\right] \mid .
\end{aligned}
$$

Now apply Lemma 2.6 to see that the second term inside the $P$-expectation is

$$
\begin{gathered}
\widetilde{P}\left[\int_{0}^{t} \int_{\mathbb{R}^{2}} \varphi_{t-s}^{\varepsilon} S_{\varepsilon} X_{t-s}^{1}(x) S_{\varepsilon} X_{t-s}^{2}(x) \tilde{L}(d s, d x)\right] \\
\quad=\lim _{\varepsilon^{\prime} \downarrow 0} \widetilde{P}\left[\int_{0}^{t} \int_{\mathbb{R}^{2}} \varphi_{t-s}^{\varepsilon} f^{\varepsilon, \varepsilon^{\prime}}(t-s, x) d x d s\right] .
\end{gathered}
$$


Similarly by applying Lemma 2.6 to the first term in (2.25) we get

$$
\begin{aligned}
I_{\varepsilon}= & 4 \gamma \mid \lim _{\varepsilon^{\prime} \downarrow 0} P\left[\int_{0}^{t} \int_{\mathbb{R}^{2}} \widetilde{P}\left[\varphi_{s}^{\varepsilon} f^{\varepsilon, \varepsilon^{\prime}}(s, x)\right] d x d s\right] \\
& -P\left[\lim _{\varepsilon^{\prime} \downarrow 0} \widetilde{P}\left[\int_{0}^{t} \int_{\mathbb{R}^{2}} \varphi_{s}^{\varepsilon} f^{\varepsilon^{\prime}, \varepsilon}(s, x) d x d s\right]\right] \mid
\end{aligned}
$$

To justify taking the limit outside the expectation in the second term, fix arbitrary $\lambda>0, \varepsilon>0$ and note that

$$
\begin{aligned}
& \widetilde{P}\left[\int_{0}^{t} \int_{\mathbb{R}^{2}}\left|\varphi_{s}^{\varepsilon}\right| f^{\varepsilon^{\prime}, \varepsilon}(s, x) d x d s\right] \\
& \leqslant \int_{0}^{t} \int_{\mathbb{R}^{2}} S_{\varepsilon} X_{s}^{1}(x) S_{\varepsilon} X_{s}^{2}(x) S_{\varepsilon^{\prime}+t-s} \widetilde{X}_{0}^{1}(x) S_{\varepsilon^{\prime}+t-s} \widetilde{X}_{0}^{2}(x) d x d s \\
& \leqslant \int_{0}^{t} \int_{\mathbb{R}^{2}} \int_{\mathbb{R}^{2}} \mathrm{p}_{\varepsilon}\left(y_{1}-x\right) \mathrm{p}_{\varepsilon}\left(y_{2}-x\right)\left(c_{A 1}(t+1), \lambda\right)^{2}\left|\widetilde{X}_{0}^{1}\right|_{\lambda}\left|\widetilde{X}_{0}^{2}\right|_{\lambda} \\
& \quad \times \phi_{2 \lambda}(x) d x X_{s}^{1}\left(d y_{1}\right) X_{s}^{2}\left(d y_{2}\right) d s \\
& \leqslant c\left(\widetilde{\mathbf{X}}_{0}, \lambda\right) \varepsilon^{-1} \int_{0}^{t} X_{s}^{1}\left(\phi_{\lambda}\right) X_{s}^{2}\left(\phi_{\lambda}\right) d s,
\end{aligned}
$$

where the first inequality follows from Lemma 2.2(c), the second one follows from Lemma A.1 and the third one from Lemma A.2. Note that $c\left(\widetilde{\mathbf{X}}_{0}, \lambda\right)$ is finite since $\widetilde{\mathbf{X}}_{0} \in\left(\mathcal{C}_{\text {rap }}\right)^{2}$.

By Lemma A.1 $S_{s} \phi_{\lambda} \in \mathcal{C}_{\lambda}$ for any $s \geqslant 0$ and any $\lambda \in \mathbb{R}$. Therefore Lemma 2.2(c) shows that $\int_{0}^{t} X_{s}^{1}\left(\phi_{\lambda}\right) X_{s}^{2}\left(\phi_{\lambda}\right) d s$ is $P$-integrable and hence we may use Dominated Convergence to write (2.26) as

$$
I_{\varepsilon}=4 \gamma\left|\lim _{\varepsilon^{\prime} \downarrow 0} P \times \widetilde{P}\left[\int_{0}^{t} \int_{\mathbb{R}^{2}} \varphi_{s}^{\varepsilon}\left(f^{\varepsilon, \varepsilon^{\prime}}(s, x)-f^{\varepsilon^{\prime}, \varepsilon}(s, x)\right) d x d s\right]\right| .
$$

Arguing as in the proof of Theorem 11(a) of [4] (this theorem establishes absolute continuity in the particular case of $X_{0}^{j} \ll d x, j=1,2$ ), we can check that the hypotheses of the general absolute continuity Theorem 57 of [3] are satisfied. Therefore, with probability $1, X_{s}^{j}$ and $\widetilde{X}_{s}^{j}$ have densities $x_{s}^{j}(\cdot)$ and $\tilde{x}_{s}^{j}(\cdot)$ respectively for $j=1,2$.

Fix $0<s<t$. Then $S_{\varepsilon} \widetilde{X}_{t-s}^{j}(x) \rightarrow \tilde{x}_{t-s}^{j}(x), j=1,2$, for Lebesgue a.a. $x, \widetilde{P}$-a.s., by standard differentiation theory. Moreover,

$$
P \times \widetilde{P}\left[\left\langle X_{s}^{j}, S_{\varepsilon} \widetilde{X}_{t-s}^{j}\right\rangle\right]
$$




$$
\begin{aligned}
& =P\left[\int_{\mathbb{R}^{2}} S_{s}\left(X_{0}^{j}\right)(x) S_{\varepsilon+t-s}\left(\tilde{X}_{0}^{j}\right)(x) d x\right] \quad \text { (by Lemma 2.2(c)) } \\
& =P\left[\iint_{\mathbb{R}^{2}} \mathrm{p}_{\mathbb{R}^{2}}\left(y_{1}-y_{2}\right) \widetilde{X}_{0}^{j}\left(d y_{1}\right) X_{0}^{j}\left(d y_{2}\right)\right] \\
& \rightarrow P\left[\iint_{\mathbb{R}^{2}} \mathrm{p}_{t}\left(y_{1}-y_{2}\right) \widetilde{X}_{0}^{j}\left(d y_{1}\right) X_{0}^{j}\left(d y_{2}\right)\right], \quad \text { as } \varepsilon \downarrow 0, j=1,2,
\end{aligned}
$$

by Dominated Convergence, Lemma A.1 and $P\left[X_{0}^{j}\left(\phi_{\lambda}\right)\right]<\infty$. Use again Lemma 2.2(c) to show

$$
\begin{aligned}
P \times \widetilde{P}\left[\int_{\mathbb{R}^{2}} \tilde{x}_{t-s}^{j}(x) X_{s}^{j}(d x)\right] & =\widetilde{P}\left[\int_{\mathbb{R}^{2}} P\left[S_{s}\left(X_{0}^{j}\right)(x)\right] \tilde{x}_{t-s}^{j}(x) d x\right] \\
& =\int_{\mathbb{R}^{2}} P\left[S_{s}\left(X_{0}^{j}\right)(x)\right] S_{t-s}\left(\widetilde{X}_{0}^{j}\right)(x) d x \\
& =P\left[\int_{\mathbb{R}^{2}} \mathrm{p}_{t}\left(y_{1}-y_{2}\right) \widetilde{X}_{0}^{j}\left(d y_{1}\right) X_{0}^{j}\left(d y_{2}\right)\right], \quad j=1,2 .
\end{aligned}
$$

It follows from (2.28) and (2.29) that

$$
S_{\varepsilon}\left(\widetilde{X}_{t-s}^{j}\right)(x) \rightarrow \tilde{x}_{t-s}^{j}(x) \quad \text { in } L^{1}\left(P \times \widetilde{P}\left(X_{s}^{j}(d x)\right)\right) \quad \text { as } \varepsilon \downarrow 0, j=1,2 \text {, }
$$

and hence

$$
\left\langle X_{s}^{j}, S_{\varepsilon}\left(\widetilde{X}_{t-s}^{j}\right)\right\rangle \rightarrow\left\langle X_{s}^{j}, \tilde{x}_{t-s}^{j}\right\rangle \quad \text { in } L^{1}(P \times \widetilde{P}) \text { as } \varepsilon \downarrow 0, j=1,2 .
$$

Therefore for each $s$ in $(0, t)$,

$$
\varphi_{s}^{\varepsilon} \rightarrow \varphi_{s} \equiv \exp \left\{-\left\langle X_{s}^{1}+X_{s}^{2}, \tilde{x}_{t-s}^{1}+\tilde{x}_{t-s}^{2}\right\rangle+i\left\langle X_{s}^{1}-X_{s}^{2}, \tilde{x}_{t-s}^{1}-\tilde{x}_{t-s}^{2}\right\rangle\right\},
$$

boundedly and a.s. as $\varepsilon \downarrow 0$. The right side is only defined up to a null set for each $s$, but clearly we may define a Borel map

$$
\hat{\varphi}: \mathcal{M}_{\text {tem }}^{4} \mapsto\{z \in \mathbb{C}:|z| \leqslant 1\}
$$

such that

$$
\varphi_{s}^{\varepsilon} \rightarrow \varphi_{s}=\hat{\varphi}\left(\mathbf{X}_{s}, \widetilde{\mathbf{X}}_{t-s}\right)
$$

boundedly and a.s. as $\varepsilon \downarrow 0$ for each $s \in(0, t)$. Our immediate goal is to prove

$$
\lim _{\varepsilon \downarrow 0} I_{\varepsilon}=0
$$


By (2.27) and an elementary argument it suffices to fix $\varepsilon_{n} \downarrow 0, \varepsilon_{n}^{\prime} \downarrow 0$ and show

$$
\lim _{n \rightarrow \infty} P \times \widetilde{P}\left[\int_{0}^{t} \varphi_{s}^{\varepsilon_{n}}\left(\int_{\mathbb{R}^{2}} f^{\varepsilon_{n}, \varepsilon_{n}^{\prime}}(s, x)-f^{\varepsilon_{n}^{\prime}, \varepsilon_{n}}(s, x) d x\right) d s\right]=0 .
$$

The key step in proving this is the following lemma:

LEMMA 2.8. -

$$
\left\{\int_{\mathbb{R}^{2}} f^{\varepsilon_{n}, \varepsilon_{n}^{\prime}}(s, x)+f^{\varepsilon_{n}^{\prime}, \varepsilon_{n}}(s, x) d x: n \in \mathbb{N}\right\}
$$

is uniformly integrable on $\Omega \times \widetilde{\Omega} \times[0, t]$ with respect to $P \times \widetilde{P} \times d s$.

We first assume this and finish the proof of (2.33) and Proposition 2.1. If $\psi: \Omega \times \widetilde{\Omega} \times$ $(0, t) \mapsto \mathbb{C}$ is bounded and $\mathcal{F} \times \widetilde{\mathcal{F}} \times \mathcal{B}(0, t)$-measurable let

$$
J_{n}(\psi)=P \times \widetilde{P}\left[\int_{0}^{t} \psi_{s}\left(\int_{\mathbb{R}^{2}} f^{\varepsilon_{n}, \varepsilon_{n}^{\prime}}(s, x)-f^{\varepsilon_{n}^{\prime}, \varepsilon_{n}}(s, x) d x\right) d s\right]
$$

(Lemma 2.2(c) easily shows this integral is finite.) The left side of (2.33) is bounded by $\limsup _{n \rightarrow \infty} P \times \widetilde{P}\left[\int_{0}^{t}\left|\varphi_{s}^{\varepsilon_{n}}-\varphi_{s}\right|\left(\int_{\mathbb{R}^{2}} f^{\varepsilon_{n}, \varepsilon_{n}^{\prime}}(s, x)+f^{\varepsilon_{n}^{\prime}, \varepsilon_{n}}(s, x) d x\right) d s\right]+\limsup _{n \rightarrow \infty} J_{n}(\varphi)$,

which by (2.34) and (2.31) is easily seen to equal $\lim \sup _{n \rightarrow \infty} J_{n}(\varphi)$. Therefore (2.32) reduces to proving

$$
\limsup _{n \rightarrow \infty} J_{n}(\varphi)=0 .
$$

Let $\Psi$ be the linear class of bounded measurable $\psi: \Omega \times \widetilde{\Omega} \times(0, t) \mapsto \mathbb{C}$ satisfying $\lim \sup _{n \rightarrow \infty} J_{n}(\psi)=0$. Assume $\psi: \Omega \times \widetilde{\Omega} \times(0, t) \mapsto \mathbb{C}$ is bounded measurable, $\left\{\psi_{k}\right\} \subset$ $\Psi$, and $\psi_{k} \rightarrow \psi$ boundedly and in $P \times \widetilde{P} \times d s$-measure. Then our uniform integrability assumption (2.34) implies

$$
\lim _{k \rightarrow \infty} \sup _{n}\left|J_{n}\left(\psi_{k}\right)-J_{n}(\psi)\right|=0
$$

which in turn shows $\psi \in \Psi$. So we have shown $\Psi$ is closed under bounded convergence in $P \times \widetilde{P} \times d s$ measure. Hence in order to prove $\varphi_{s}=\hat{\varphi}\left(\mathbf{X}_{s}, \widetilde{\mathbf{X}}_{t-s}\right) \in \Psi$, we may assume $\hat{\varphi}$ is bounded and continuous. Now we may approximate $\varphi_{s}$ by the appropriate sequence of step functions and use the linearity of $\Psi$, to see that it suffices to prove (2.35) for

$$
\varphi_{s}=\hat{\varphi}\left(\mathbf{X}_{a}, \widetilde{\mathbf{X}}_{t-a-\delta_{3}}\right) 1\left(a+\delta_{1} \leqslant s<a+\delta_{2}\right) \equiv \varphi_{a} 1_{\left[a+\delta_{1}, a+\delta_{2}\right)}(s),
$$


where $a<a+\delta_{1}<a+\delta_{2}<a+\delta_{3} \leqslant t$ and $\hat{\varphi}$ is bounded continuous. Fix such a $\varphi$ and note by Fubini's theorem $\left(\mathcal{F}_{a}^{X}=\sigma\left(\mathbf{X}_{s}: s \leqslant a+\right)\right)$

$$
\begin{aligned}
J_{n}(\varphi)= & P \times \widetilde{P}\left[\varphi_{a} \int_{a+\delta_{1}}^{a+\delta_{2}} \int P\left[S_{\mathbb{R}_{n}^{2}}\left(X_{s}^{1}\right)(x) S_{\varepsilon_{n}^{\prime}}\left(X_{s}^{2}\right)(x) \mid \mathcal{F}_{a}^{X}\right]\right. \\
& \times P\left[S_{\varepsilon_{n}}\left(\widetilde{X}_{t-s}^{1}\right)(x) S_{\varepsilon_{n}}\left(\widetilde{X}_{t-s}^{2}\right)(x) \mid \mathcal{F}_{t-a-\delta_{3}}^{\widetilde{\mathbf{x}}}\right] \\
& -P\left[S_{\varepsilon_{n}}\left(X_{s}^{1}\right)(x) S_{\varepsilon_{n}}\left(X_{s}^{2}\right)(x) \mid \mathcal{F}_{a}^{X}\right] \\
& \left.\left.\times P\left[S_{\varepsilon_{n}^{\prime}}\left(\widetilde{X}_{t-s}^{1}\right)(x) S_{\varepsilon_{n}^{\prime}}\left(\widetilde{X}_{t-s}^{2}\right)(x) \mid \mathcal{F}_{t-a-\delta_{3}}^{\widetilde{\mathbf{x}}}\right]\right) d x d s\right] \\
= & P \times \widetilde{P}\left[\varphi _ { a } \int _ { a + \delta _ { 1 } } ^ { a + \delta _ { 2 } } \left(S _ { \mathbb { R } ^ { 2 } } \left({\widetilde{\varepsilon_{n}^{\prime}+s-a}}_{1}\left(X_{a}^{1}\right)(x) S_{\varepsilon_{n}^{\prime}+s-a}\left(X_{a}^{2}\right)(x)\right.\right.\right. \\
& \times S_{\varepsilon_{n}+a+\delta_{3}-s}\left(\widetilde{X}_{t-a-\delta_{3}}^{1}\right)(x) S_{\varepsilon_{n}+a+\delta_{3}-s}\left(\widetilde{X}_{t-a-\delta_{3}}^{2}\right)(x) \\
& -S_{\varepsilon_{n}+s-a}\left(X_{a}^{1}\right)(x) S_{\varepsilon_{n}+s-a}\left(X_{a}^{2}\right)(x) \\
& \left.\left.\times S_{\varepsilon_{n}^{\prime}+a+\delta_{3}-s}\left(\widetilde{X}_{t-a-\delta_{3}}^{1}\right)(x) S_{\varepsilon_{n}^{\prime}+a+\delta_{3}-s}\left(\widetilde{X}_{t-a-\delta_{3}}^{2}\right)(x)\right) d x d s\right],
\end{aligned}
$$

where we have used Lemma 2.2(c) in the last line. Note that the integrand in brackets approaches 0 as $n \rightarrow \infty$ (note $s-a \geqslant \delta_{1}>0$ and $a-s+\delta_{3} \geqslant \delta_{3}-\delta_{2}>0$ ). Fix $\lambda_{1}>\lambda_{2}>0$ and use Corollary A.4 to bound the integrand by

$$
\begin{aligned}
& c_{A 3}\left(t+1, \lambda_{1}\right)^{2} c_{A 3}\left(t+1, \lambda_{2}\right)^{2} c_{A 4}\left(\delta_{1} \wedge\left(\delta_{3}-\delta_{2}\right)\right)^{4} X_{a}^{1}\left(\phi_{\lambda_{2}}\right) X_{a}^{2}\left(\phi_{\lambda_{2}}\right) \\
& \quad \times \widetilde{X}_{t-a-\delta_{3}}^{1}\left(\phi_{-\lambda_{1}}\right) \widetilde{X}_{t-a-\delta_{3}}^{2}\left(\phi_{-\lambda_{1}}\right) \phi_{2\left(\lambda_{1}-\lambda_{2}\right)}(x)
\end{aligned}
$$

(without loss of generality we assume that $\varepsilon_{n}, \varepsilon_{n}^{\prime}<1$ ). By Lemma 2.2(c) and our assumptions on the initial conditions $\mathbf{X}_{0}$ and $\widetilde{\mathbf{X}}_{0}$, this latter expression is integrable with respect to $P \times \widetilde{P} \times d x \times d s$ and so by Dominated Convergence we have $\lim _{n \rightarrow \infty} J_{n}(\varphi)=$ 0 . This completes the proof of (2.32).

It is now easy to use (2.32) to prove Proposition 2.1. As $\widetilde{X}_{0}^{j}$ has a bounded continuous density $\tilde{x}_{0}^{j}(\cdot), S_{\varepsilon}\left(\tilde{X}_{0}^{j}\right)(x) \rightarrow \tilde{x}_{0}^{j}$ pointwise boundedly as $\varepsilon \downarrow 0$ and so by Lemma A.1 and Dominated Convergence $\left\langle X_{s}^{j}, S_{\varepsilon} \tilde{X}_{0}^{j}\right\rangle \rightarrow\left\langle X_{s}^{j}, \tilde{x}_{0}^{j}\right\rangle$. A second application of Dominated Convergence now shows $\left(D_{1}\right)$ is immediate from (2.32).

It remains only to prove the uniform integrability condition (2.34). To this end we need the following moment condition on $\widetilde{\mathbf{X}}$ which will be also used in the proof of Theorem 1.11(a).

Lemma 2.9. - Let $\gamma / \sigma^{2}<\left(\sqrt{6} \pi c_{\mathrm{rw}}\right)^{-1}, \widetilde{\mathbf{X}}_{0} \in\left(\mathcal{B}_{\mathrm{b}}^{+}\right)^{2}$ and $\widetilde{\mathbf{X}}$ be the particular solution to (MP) $\widetilde{\mathbf{x}}_{0}^{\sigma, \gamma}$ constructed in Section 1.4. Define

$$
w_{t}^{\eta}(x, y)=\widetilde{P}\left[S_{\eta}\left(\widetilde{X}_{t}^{1}\right)(x) S_{\eta}\left(\widetilde{X}_{t}^{2}\right)(x) S_{\eta}\left(\widetilde{X}_{t}^{1}\right)(y) S_{\eta}\left(\widetilde{X}_{t}^{2}\right)(y)\right], \quad\left(\eta, t>0 ; x, y \in \mathbb{R}^{2}\right) .
$$


For any $p: 0<p \leqslant 1 / 2$ such that

$$
\gamma / \sigma^{2}<\frac{\sin (\pi(1-p))}{\sqrt{6} \pi c_{\mathrm{rw}}}
$$

the following holds. For each $T>0$, there is a $c_{T}=c_{T}\left(\gamma, \sigma^{2}, p, \widetilde{\mathbf{X}}_{0}\right)>0$ such that

$$
w_{t}^{\eta}(x, y) \leqslant c_{T}\left(1+|x-y|^{-2 p}\right), \quad \forall \eta \in(0,1], x, y \in \mathbb{R}^{2}, 0 \leqslant t \leqslant T .
$$

Proof. - Recall that $\widetilde{\mathbf{X}}=\left(\widetilde{X}^{1}, \widetilde{X}^{2}\right)$ is a weak limit point in $C\left(\mathbb{R}_{+}, \mathcal{M}_{\text {tem }}^{2}\right)$ of a sequence of rescaled lattice systems ${ }^{\varepsilon_{n}} \widetilde{\mathbf{X}}=\left({ }^{\varepsilon_{n}} \widetilde{X}^{1},{ }^{\varepsilon_{n}} \widetilde{X}^{2}\right)$. By taking subsequence if necessary we may assume that $\widetilde{\mathbf{X}}$ is the limit in law of ${ }^{\varepsilon_{n}} \widetilde{\mathbf{X}}$. Recall (see Section 1.4) that ${ }^{\varepsilon} \mathcal{M}_{\text {tem }}$ is the subspace of $\mathcal{M}_{\text {tem }}$ of measures with densities with respect to $d^{\varepsilon} x$. Let

$$
w_{t}^{\eta, \varepsilon}(x, y)=\widetilde{P}\left[{ }^{\varepsilon} S_{\eta}\left({ }^{\varepsilon} \widetilde{X}_{t}^{1}\right)(x)^{\varepsilon} S_{\eta}\left({ }^{\varepsilon} \widetilde{X}_{t}^{2}\right)(x)^{\varepsilon} S_{\eta}\left({ }^{\varepsilon} \widetilde{X}_{t}^{1}\right)(y)^{\varepsilon} S_{\eta}\left({ }^{\varepsilon} \widetilde{X}_{t}^{2}\right)(y)\right], \quad x, y \in \varepsilon \mathbb{Z}^{2},
$$

where

$$
{ }^{\varepsilon} S_{\eta}(\mu)(x) \equiv \int_{\mathbb{R}^{2}} \mathrm{p}_{\eta}(x-y) \mu(d y), \quad \forall x \in \varepsilon \mathbb{Z}^{2}, \forall \mu \in{ }^{\varepsilon} \mathcal{M}_{\mathrm{tem}}, \eta>0 .
$$

A local central limit theorem (see Lemma 8(a)) of [3] implies

$$
d(\varepsilon, \eta) \equiv \sup _{x \in \varepsilon \mathbb{Z}^{2}}\left|{ }^{\varepsilon} \mathrm{p}_{\eta}(x)-\mathrm{p}_{\eta}(x)\right| \rightarrow 0, \quad \text { as } \varepsilon \downarrow 0 \text { for each } \eta>0 .
$$

By Skorohod's Theorem we may assume ${ }^{\varepsilon_{n}} \widetilde{\mathbf{X}}_{t} \rightarrow \widetilde{\mathbf{X}}_{t}$ in $\mathcal{M}_{\text {tem }}^{2}$ a.s. Fix arbitrary $x \in \mathbb{R}^{2}$ and a sequence $\left\{x_{n}\right\}$ such that $x_{n} \in \varepsilon_{n} \mathbb{Z}^{2}, \forall n \geqslant 1$ and $\lim _{n \rightarrow \infty} x_{n}=x$. Then, for any compact set $K \subset \mathbb{R}^{2}$,

$$
\begin{aligned}
& \underset{n \rightarrow \infty}{\limsup _{n \rightarrow \infty}}\left|{ }^{\varepsilon_{n}} S_{\eta}\left({ }^{\varepsilon_{n}} \widetilde{X}_{t}^{j}\right)\left(x_{n}\right)-S_{\eta}\left(\widetilde{X}_{t}^{j}\right)(x)\right| \\
& \leqslant \limsup _{n \rightarrow \infty} \int_{K}\left|{ }^{\varepsilon_{n}} \mathrm{p}_{\eta}\left(z-x_{n}\right)-\mathrm{p}_{\eta}(z-x)\right|^{\varepsilon_{n}} \widetilde{X}_{t}^{j}(d z) \\
& \quad+\limsup _{n \rightarrow \infty} \int_{K^{c}}\left|{ }^{\varepsilon_{n}} \mathrm{p}_{\eta}\left(z-x_{n}\right)-\mathrm{p}_{\eta}(z-x)\right|^{\varepsilon_{n}} \widetilde{X}_{t}^{j}(d z) \\
& \quad+\limsup _{n \rightarrow \infty}\left|\int_{\mathbb{R}^{2}} \mathrm{p}_{\eta}(z-x)\left({ }^{\varepsilon_{n}} \widetilde{X}_{t}^{j}(d z)-\widetilde{X}_{t}^{j}(d z)\right)\right| \\
& =\limsup _{n \rightarrow \infty} \int_{K^{c}}\left|{ }^{\varepsilon_{n}} \mathrm{p}_{\eta}\left(z-x_{n}\right)-\mathrm{p}_{\eta}(z-x)\right|^{\varepsilon_{n}} \widetilde{X}_{t}^{j}(d z) \\
& \equiv \limsup _{n \rightarrow \infty} I_{K}^{n}, \quad j=1,2,
\end{aligned}
$$

by (2.37) and the above a.s. convergence ${ }^{\varepsilon_{n}} \widetilde{\mathbf{X}}_{t} \rightarrow \widetilde{\mathbf{X}}_{t}$ in $\mathcal{M}_{\text {tem }}^{2}$. 
For any $\varepsilon>0$, there exists a compact set $K_{\varepsilon} \subset \mathbb{R}^{2}$ such that

$$
\begin{aligned}
\widetilde{P}\left[I_{K_{\varepsilon}}^{n}\right] & =\int_{K_{\varepsilon}^{c}}\left|{ }^{\varepsilon_{n}} \mathrm{p}_{\eta}\left(z-x_{n}\right)-\mathrm{p}_{\eta}(z-x)\right| \widetilde{P}\left[{ }^{\varepsilon_{n}} \widetilde{X}_{t}^{j}(d z)\right] \\
& \leqslant\left\|\widetilde{X}_{0}^{j}\right\|_{\infty} \int_{K_{\varepsilon}^{c}}\left|{ }^{\varepsilon_{n}} \mathrm{p}_{\eta}\left(z-x_{n}\right)-\mathrm{p}_{\eta}(z-x)\right| d^{\varepsilon_{n}} z \leqslant \varepsilon,
\end{aligned}
$$

uniformly in $n$. Since $\varepsilon$ was arbitrary we get by (2.38) that ${ }^{\varepsilon_{n}} S_{\eta}\left({ }^{\varepsilon_{n}} \widetilde{X}_{t}^{j}\right)\left(x_{n}\right) \rightarrow S_{\eta}\left(\widetilde{X}_{t}^{j}\right)(x)$ in probability, as $n \rightarrow \infty$ for $j=1,2$.

Now Fatou's Lemma shows that for arbitrary $x, y \in \mathbb{R}^{2}$

$$
w_{t}^{\eta}(x, y) \leqslant \liminf _{n \rightarrow \infty} w_{t}^{\eta, \varepsilon_{n}}\left(x_{n}, y_{n}\right),
$$

where $\lim _{n \rightarrow \infty} x_{n}=x, \lim _{n \rightarrow \infty} y_{n}=y$ and $x_{n}, y_{n} \in \varepsilon_{n} \mathbb{Z}^{2}, \forall n \geqslant 1$.

For any $\varepsilon>0$ let ${ }^{\varepsilon} \vec{p}_{\eta}\left(z_{1}, z_{2}, z_{3}, z_{4}\right)=\prod_{i=1}^{4}{ }^{\varepsilon} \mathrm{p}_{\eta}\left(z_{i}\right)$ be the transition function for the 8 -dimensional continuous time simple symmetric random walk on $\varepsilon \mathbb{Z}^{8}$. Recall that

$$
{ }^{\varepsilon} m_{t}^{1122}(\vec{z})=\widetilde{P}\left[{ }^{\varepsilon} \widetilde{X}_{t}^{1}\left(z_{1}\right)^{\varepsilon} \widetilde{X}_{t}^{1}\left(z_{2}\right)^{\varepsilon} \widetilde{X}_{t}^{2}\left(z_{3}\right)^{\varepsilon} \widetilde{X}_{t}^{2}\left(z_{4}\right)\right],
$$

and hence

$$
w_{t}^{\eta, \varepsilon}(x, y)=\int_{\mathbb{R}^{8}} \varepsilon \vec{p}_{\eta}(\vec{z}-(x, y, x, y))^{\varepsilon} m_{t}^{1122}(\vec{z}) d^{\varepsilon} \vec{z}
$$

where $d^{\varepsilon} \vec{z}$ assigns mass $\varepsilon^{8}$ to each point in $\left(\varepsilon \mathbb{Z}^{2}\right)^{4}$. Apply (1.13) and ChapmanKolmogorov to conclude that for $0 \leqslant t \leqslant T$,

$$
w_{t}^{\eta, \varepsilon}(x, y) \leqslant c_{T}\left(\gamma, \sigma^{2}, p, \widetilde{\mathbf{X}}_{0}\right)\left(1+\int_{0}^{t} u^{-p}\left({ }^{\varepsilon} \mathrm{p}_{2(u+\eta)}(x-y)\right) d u\right) .
$$

Now use (2.37), (2.39) and Dominated Convergence to conclude that for $\eta \in(0,1]$ and $p$ as in (2.36),

$$
\begin{aligned}
w_{t}^{\eta}(x, y) \leqslant & c_{T}\left(\gamma, \sigma^{2}, p, \widetilde{\mathbf{X}}_{0}\right)\left(1+\int_{0}^{t} u^{-p} \mathrm{p}_{2(u+\eta)}(x-y) d u\right) \\
\leqslant & c_{T}\left(\gamma, \sigma^{2}, p, \widetilde{\mathbf{X}}_{0}\right)\left(1+\int_{0}^{\eta} u^{-p} d u 2 \mathrm{p}_{4 \eta}(x-y)\right. \\
& \left.+\int_{\eta}^{t} 2^{p}(u+\eta)^{-p} 2 \mathrm{p}_{2(u+\eta)}(x-y) d u\right) \\
\leqslant & c_{T}\left(\gamma, \sigma^{2}, p, \widetilde{\mathbf{X}}_{0}\right)\left(1+\eta^{-p} \exp \left\{-\frac{|x-y|^{2}}{8 \eta}\right\}+\int_{0}^{t+1} u^{-p} \mathrm{p}_{2 u}(x-y) d u\right)
\end{aligned}
$$




$$
\leqslant c_{T}\left(\gamma, \sigma^{2}, p, \widetilde{\mathbf{X}}_{0}\right)\left(1+|x-y|^{-2 p}\right), \quad \forall 0 \leqslant t \leqslant T .
$$

In the last line we use

$$
\eta^{-p} \mathrm{e}^{-\Delta^{2} / 8 \eta} \leqslant \sup _{z} z^{2 p} \mathrm{e}^{-z^{2} / 8} \Delta^{-2 p}
$$

to bound the term preceding the integral and the substitution $w=|x-y|^{2} / 8 u$ to handle the integral. This gives the desired bound.

COROllary 2.10. - Let $\gamma \sigma^{-2}, \widetilde{\mathbf{X}}_{0}$ and $\widetilde{\mathbf{X}}$ be as in Lemma 2.9. For each $T>0$, there is a $c_{T}=c_{T}\left(\gamma, \sigma^{2}, \widetilde{\mathbf{X}}_{0}\right)>0$ such that

$$
w_{t}^{\eta}(x, y) \leqslant c_{T}\left(1+|x-y|^{-1}\right), \quad \forall \eta \in(0,1], x, y \in \mathbb{R}^{2}, t \in[0, T] .
$$

Proof. - Immediately from the previous lemma with $p=1 / 2$.

Corollary 2.11. - Let $\gamma \sigma^{-2}, \widetilde{\mathbf{X}}_{0}$ and $\widetilde{\mathbf{X}}$ be as in Lemma 2.9. Then $\widetilde{\mathbf{X}}$ satisfies (SIntC).

Proof. - Take $p<1 / 2$ satisfying (2.36). Then, for any compact set $K \subset \mathbb{R}^{2}$, we have for $s \leqslant T$,

$$
\begin{aligned}
P\left[H_{\varepsilon, K}\left(\widetilde{\mathbf{X}}_{s}\right)\right] & =\int_{K} \int_{K}\left(1+|x-y|^{-1}\right) w_{s}^{\varepsilon}(x, y) d x d y \\
& \leqslant \int_{K} \int_{K}\left(1+|x-y|^{-1}\right) c_{T}\left(1+|x-y|^{-2 p}\right) d x d y \quad \text { (by Lemma 2.9) } \\
& <\infty
\end{aligned}
$$

uniformly in $0<\varepsilon<1$. This gives the desired result.

Remark 2.12. - The proof of Theorem 1.11(a) is now finished since Corollary 2.11 implies that solutions constructed in [4] satisfy (SIntC).

Proof of Lemma 2.8. - As $\varepsilon_{n}, \varepsilon_{n}^{\prime} \downarrow 0$ are arbitrary we only need to show $\left\{\int_{\mathbb{R}^{2}} f^{\varepsilon_{n}, \varepsilon_{n}^{\prime}}(s\right.$, $x) d x: n \in \mathbb{N}\}$ are uniformly integrable with respect to $P \times \widetilde{P} \times d s$. As a first step we will show that for any compact set $K \subset \mathbb{R}^{2}$,

$$
\left\{\int_{K} f^{\varepsilon_{n}, \varepsilon_{n}^{\prime}}(s, x) d x: n \in \mathbb{N}\right\} \text { is uniformly integrable with respect to } P \times \widetilde{P} \times d s .
$$

This would follow from (each of the above functions are integrable by Lemma 2.2(c))

$$
\sup _{n \geqslant n_{0}} P \times \widetilde{P}\left[\int_{0}^{t}\left(\int_{K} f^{\varepsilon_{n}, \varepsilon_{n}^{\prime}}(s, x) d x\right)^{2} d s\right]<\infty, \quad \text { for some } n_{0} \in \mathbb{N} .
$$

For fixed $n$ the above expectation is

$$
\int_{0}^{t} \int_{K} \int_{K} \widetilde{P}\left[S_{\varepsilon_{n}}\left(\widetilde{X}_{t-s}^{1}\right)(x) S_{\varepsilon_{n}}\left(\widetilde{X}_{t-s}^{2}\right)(x) S_{\varepsilon_{n}}\left(\widetilde{X}_{t-s}^{1}\right)(y) S_{\varepsilon_{n}}\left(\widetilde{X}_{t-s}^{2}\right)(y)\right]
$$




$$
\begin{aligned}
& \times P\left[S_{\varepsilon_{n}^{\prime}}\left(X_{s}^{1}\right)(x) S_{\varepsilon_{n}^{\prime}}\left(X_{s}^{2}\right)(x) S_{\varepsilon_{n}^{\prime}}\left(X_{s}^{1}\right)(y) S_{\varepsilon_{n}^{\prime}}\left(X_{s}^{2}\right)(y)\right] \\
\leqslant & c_{t} P\left(\int_{0}^{t} H_{\varepsilon_{n}^{\prime}, K}\left(\mathbf{X}_{s}\right) d s\right) \quad(\text { by Corollary 2.10). }
\end{aligned}
$$

By (SIntC), there are constants $n_{0} \in \mathbb{N}$ and $c_{t, K}^{\prime}>0$ such that

$$
\sup _{n \geqslant n_{0}} c_{t} \int_{0}^{t} H_{\varepsilon_{n}^{\prime}, K}\left(\mathbf{X}_{s}\right) d s \leqslant c_{t, K}^{\prime} .
$$

This gives (2.41) and so (2.40).

Fix arbitrary $\lambda>0$ and assume without loss of generality that $\varepsilon_{n}, \varepsilon_{n}^{\prime}<1$. Now by Lemma 2.2(c), Lemma A.1 and simple calculus we get

$$
\begin{aligned}
& \int_{\mathbb{R}^{2}} \int_{0}^{t} \widetilde{P} \times P\left[f^{\varepsilon_{n}, \varepsilon_{n}^{\prime}}(s, x)\right] d s d x \\
& \quad=\int_{\mathbb{R}^{2}} \int_{0}^{t} \widetilde{P}\left[S_{t-s+\varepsilon_{n}}\left(\widetilde{X}_{0}^{1}\right)(x) S_{t-s+\varepsilon_{n}}\left(\widetilde{X}_{0}^{2}\right)(x)\right] P\left[S_{s+\varepsilon_{n}^{\prime}}\left(X_{0}^{1}\right)(x) S_{s+\varepsilon_{n}^{\prime}}\left(X_{0}^{2}\right)(x)\right] d s d x \\
& \quad \leqslant\left(c_{A 1}(t+1), \lambda\right)^{2}\left|\widetilde{X}_{0}^{1}\right|_{\lambda}\left|\widetilde{X}_{0}^{2}\right|_{\lambda} \int_{\mathbb{R}^{2}} \int_{0}^{t} \phi_{2 \lambda}(x) P\left[S_{s+\varepsilon_{n}^{\prime}}\left(X_{0}^{1}\right)(x) S_{s+\varepsilon_{n}^{\prime}}\left(X_{0}^{2}\right)(x)\right] d s d x \\
& \quad \leqslant\left.\left|\left(c_{A 1}(t+1), \lambda\right)^{2}\right| \widetilde{X}_{0}^{1}\right|_{\lambda}\left|\widetilde{X}_{0}^{2}\right|_{\lambda} \int_{\mathbb{R}^{2}}^{t+1} \int_{0}^{2} \phi_{2 \lambda}(x) P\left[\bar{p}_{s}\left(X_{0}^{1}, X_{0}^{2}\right)(x)\right] d s d x \\
& \quad=\left(c_{A 1}(t+1), \lambda\right)^{2}\left|\widetilde{X}_{0}^{1}\right|_{\lambda}\left|\widetilde{X}_{0}^{2}\right|_{\lambda} \int_{\mathbb{R}^{2}} \phi_{2 \lambda}(x) P\left[\bar{g}_{t+1}\left(X_{0}^{1}, X_{0}^{2}\right)(x)\right] d x, \quad \forall n \geqslant 1 .
\end{aligned}
$$

The last bound is finite by (EnC) condition and hence, by Fubini's theorem, we get

$$
\sup _{n} \widetilde{P} \times P\left[\int_{0}^{t} \int_{\mathbb{R}^{2}} f^{\varepsilon_{n}, \varepsilon_{n}^{\prime}}(s, x) d x d s\right]<\infty .
$$

Moreover, for any $n \geqslant 1, \int_{0}^{t} \widetilde{P} \times P\left[f^{\varepsilon_{n}, \varepsilon_{n}^{\prime}}(s, x)\right] d s$ is dominated by integrable function

$$
\left(c_{A 1}(t+1), \lambda\right)^{2}\left|\widetilde{X}_{0}^{1}\right|_{\lambda}\left|\widetilde{X}_{0}^{2}\right|_{\lambda} \phi_{2 \lambda}(x) P\left[\bar{g}_{t+1}\left(X_{0}^{1}, X_{0}^{2}\right)(x)\right] .
$$

Therefore for arbitrary $\delta>0$ we can fix a compact set $K \subset \mathbb{R}^{2}$ such that

$$
\int_{0}^{t} \int_{K^{c}} \widetilde{P} \times P\left[f^{\varepsilon_{n}, \varepsilon_{n}^{\prime}}(s, x)\right] d x d s \leqslant \delta / 2, \quad \forall n \geqslant 1 .
$$


It follows from (2.42) that $\left\{\int_{\mathbb{R}^{2}} f^{\varepsilon_{n}, \varepsilon_{n}^{\prime}}(s, x) d x\right\}$ is $\widetilde{P} \times P \times d s$-tight, and so we can choose $N$ such that

$$
\int_{0}^{t} \widetilde{P} \times P\left(\int_{\mathbb{R}^{2}} f^{\varepsilon_{n}, \varepsilon_{n}^{\prime}}(s, x) d x \geqslant N\right) d s \leqslant \delta^{2} / 4 c_{t, K}^{\prime}
$$

for all $n$ sufficiently large. Therefore

$$
\begin{aligned}
\widetilde{P} & \times P\left[\int_{0}^{t}\left(\int_{\mathbb{R}^{2}} f^{\varepsilon_{n}, \varepsilon_{n}^{\prime}}(s, x) d x\right) 1\left(\int_{\mathbb{R}^{2}} f^{\varepsilon_{n}, \varepsilon_{n}^{\prime}}(s, x) d x \geqslant N\right) d s\right] \\
\leqslant & \widetilde{P} \times P\left[\int_{0}^{t}\left(\int_{K} f^{\varepsilon_{n}, \varepsilon_{n}^{\prime}}(s, x) d x\right) 1\left(\int_{\mathbb{R}^{2}} f^{\varepsilon_{n}, \varepsilon_{n}^{\prime}}(s, x) d x \geqslant N\right) d s\right] \\
& +\widetilde{P} \times P\left[\int_{0}^{t}\left(\int_{K^{c}} f^{\varepsilon_{n}, \varepsilon_{n}^{\prime}}(s, x) d x\right) 1\left(\int_{\mathbb{R}^{2}} f^{\varepsilon_{n}, \varepsilon_{n}^{\prime}}(s, x) d x \geqslant N\right) d s\right] \\
\leqslant & \sqrt{\int_{0}^{t} \widetilde{P} \times P\left[\left(\int_{K} f^{\varepsilon_{n}, \varepsilon_{n}^{\prime}}(s, x) d x\right)^{2}\right] d s} \\
& \times \sqrt{\int_{0}^{t} \widetilde{P} \times P\left(\int_{\mathbb{R}^{2}} f^{\varepsilon_{n}, \varepsilon_{n}^{\prime}}(s, x) d x \geqslant N\right) d s}+\delta / 2 \\
\leqslant & \delta .
\end{aligned}
$$

This gives (2.34) and so completes the proof of Proposition 2.1.

Proof of Proposition 1.12. - Fix $t>0$ and any $\delta \in(0, t)$. (IntC) implies that there exists a sequence $F_{1} \subset F_{2} \subset \cdots$ of events in $\mathcal{F}_{\delta}$ such that $F_{l} \uparrow \Omega$, as $l \rightarrow \infty$ and for each $n \geqslant 1$ :

$$
\underset{\varepsilon \downarrow 0}{\limsup P}\left[\int_{\delta}^{t} H_{\varepsilon, K}\left(\mathbf{X}_{s}\right) d s 1_{F_{n}}\right]<\infty, \quad \forall \text { compact set } K \subset \mathbb{R}^{2}, \forall t \geqslant \delta .
$$

Define the process $\widehat{\mathbf{X}}_{t}^{l}=\mathbf{X}_{\delta+t}$, filtration $\mathcal{F}_{t}^{\delta} \equiv \mathcal{F}_{\delta+t}$ and

$$
P_{l}(B)=P\left(1_{F_{l}} P\left(1_{B} \mid \mathcal{F}_{\delta}\right)\right) / P\left(F_{l}\right), \quad B \in \mathcal{F} .
$$

Then $\widehat{\mathbf{X}}_{t}^{l}$ satisfies the $(\mathbf{M P})_{\widehat{\mathbf{X}}_{0}^{l}}^{\sigma, \gamma}$ on $\left(\Omega, \mathcal{F}, \mathcal{F}_{t}^{\delta}, P_{l}\right)$ with

$$
v^{l}(\cdot)=\mathcal{L}\left(\widehat{\mathbf{X}}_{0}^{l}\right)=P\left(\mathbf{X}_{\delta} \in \cdot \mid F_{l}\right) .
$$


Note that

$$
\underset{\varepsilon \downarrow 0}{\limsup } P_{l}\left[\int_{0}^{t} H_{\varepsilon, K}\left(\widehat{\mathbf{X}}_{0}^{l}\right) d s\right]=\underset{\varepsilon \downarrow 0}{\limsup P}\left[\int_{\delta}^{t+\delta} H_{\varepsilon, K}\left(\mathbf{X}_{s}\right) d s 1_{F_{l}}\right] / P\left(F_{l}\right)<\infty,
$$

for any compact set $K \subset \mathbb{R}^{2}$. Therefore $\widehat{\mathbf{X}}^{l}$ satisfies (SIntC). (EnC) for $\widehat{\mathbf{X}}_{0}^{l}$ also follows easily by Lemma 2.2. Therefore Proposition 2.1 shows that for any $t \geqslant 0$,

$$
P_{l}\left[\mathfrak{E}\left(\widehat{\mathbf{X}}_{t}^{l}, \tilde{x}_{0}\right)\right]=\lim _{\varepsilon \downarrow 0} P_{l} \times \widetilde{P}\left[\mathfrak{E}\left(\widehat{\mathbf{X}}_{0}^{l}, S_{\varepsilon}\left(\widetilde{\mathbf{X}}_{t}\right)\right)\right] .
$$

Now let us take $l \rightarrow \infty$. We immediately get that

$$
\lim _{l \rightarrow \infty} P_{l}\left[\mathfrak{E}\left(\widehat{\mathbf{X}}_{t}^{l}, \tilde{x}_{0}\right)\right]=P\left[\mathfrak{E}\left(\mathbf{X}_{t+\delta}, \tilde{x}_{0}\right)\right] .
$$

For the right hand side we have

$$
\begin{aligned}
\left|\lim _{l \rightarrow \infty} \lim _{\varepsilon \downarrow 0} P_{l} \times \widetilde{P}\left[\mathfrak{E}\left(\widehat{\mathbf{X}}_{0}^{l}, S_{\varepsilon}\left(\widetilde{\mathbf{X}}_{t}\right)\right)\right]-\lim _{\varepsilon \downarrow 0} P \times \widetilde{P}\left[\mathfrak{E}\left(\mathbf{X}_{\delta}, S_{\varepsilon}\left(\widetilde{\mathbf{X}}_{t}\right)\right)\right]\right| \\
=\mid \lim _{l \rightarrow \infty} \lim _{\varepsilon \downarrow 0} \int_{\Omega} \int_{\widetilde{\Omega}} \mathfrak{E}\left(\mathbf{X}_{\delta}, S_{\varepsilon}\left(\widetilde{\mathbf{X}}_{t}\right)\right) 1_{F_{l}}(\omega) / P\left(F_{l}\right) P(d \omega) \widetilde{P}(d \tilde{\omega}) \\
\quad-\lim _{\varepsilon \downarrow 0} \int_{\Omega} \int_{\widetilde{\Omega}} \mathfrak{E}\left(\mathbf{X}_{\delta}, S_{\varepsilon}\left(\widetilde{\mathbf{X}}_{t}\right)\right) P(d \omega) \widetilde{P}(d \tilde{\omega}) \mid \\
\leqslant \lim _{l \rightarrow \infty} \lim _{\varepsilon \downarrow 0} \int_{\Omega} \int_{\widetilde{\Omega}} \mathfrak{E}\left(\mathbf{X}_{\delta}, S_{\varepsilon}\left(\widetilde{\mathbf{X}}_{t}\right)\right)\left|1_{F_{l}}(\omega) / P\left(F_{l}\right)-1\right| P(d \omega) \widetilde{P}(d \tilde{\omega}) \\
\leqslant \lim _{l \rightarrow \infty} \int_{\Omega}\left|1_{F_{l}}(\omega) / P\left(F_{l}\right)-1\right| P(d \omega) \\
=0,
\end{aligned}
$$

where the last limit follows by Bounded Convergence Theorem. Therefore we have

$$
P\left[\mathfrak{E}\left(\mathbf{X}_{t+\delta}, \tilde{x}_{0}\right)\right]=\lim _{\varepsilon \downarrow 0} P \times \widetilde{P}\left[\mathfrak{E}\left(\mathbf{X}_{\delta}, S_{\varepsilon}\left(\widetilde{\mathbf{X}}_{t}\right)\right)\right], \quad \forall t, \delta>0 .
$$

Now we have to let $\delta \downarrow 0$. By continuity of $\mathbf{X}$, the left hand side converges to $P\left[\mathfrak{E}\left(\mathbf{X}_{t}, \tilde{x}_{0}\right)\right]$ and we have to handle interchange of limits on the right hand side. By Lemma 2.3(b), for any $\delta, \varepsilon>0$, we have

$$
\begin{aligned}
P & \times \widetilde{P}\left[\mathfrak{E}\left(\mathbf{X}_{\delta}, S_{\varepsilon}\left(\widetilde{\mathbf{X}}_{t}\right)\right)\right] \\
= & P \times \widetilde{P}\left[\mathfrak{E}\left(\mathbf{X}_{0}, S_{\varepsilon+\delta}\left(\widetilde{\mathbf{X}}_{t}\right)\right)\right] \\
& +P \times \widetilde{P}\left[\int_{0}^{\delta} \mathfrak{E}\left(\mathbf{X}_{s}, S_{\varepsilon+\delta-s}\left(\widetilde{\mathbf{X}}_{t}\right)\right) 4 \gamma S_{\varepsilon+\delta-s}\left(\widetilde{X}_{t}^{1}\right)(x) S_{\varepsilon+\delta-s}\left(\widetilde{X}_{t}^{2}\right)(x) L(d s, d x)\right] \\
& \equiv I_{\varepsilon, \delta}^{1}+I_{\varepsilon, \delta}^{2} .
\end{aligned}
$$


Trivially

$$
\lim _{\delta \downarrow 0} \lim _{\varepsilon \downarrow 0} I_{\varepsilon, \delta}^{1}=\lim _{\varepsilon \downarrow 0} P \times \widetilde{P}\left[\mathfrak{E}\left(\mathbf{X}_{0}, S_{\varepsilon}\left(\widetilde{\mathbf{X}}_{t}\right)\right)\right] .
$$

We will show that $\lim _{\delta \downarrow 0} \lim _{\varepsilon \downarrow 0} I_{\varepsilon, \delta}^{2}=0$. Without loss of generality assume that $\varepsilon+\delta<1$.

$$
\begin{aligned}
\left|I_{\varepsilon, \delta}^{2}\right| \leqslant & 4 \gamma P \times \widetilde{P}\left[\int_{0}^{\delta} S_{\varepsilon+\delta-s}\left(\widetilde{X}_{t}^{1}\right)(x) S_{\varepsilon+\delta-s}\left(\widetilde{X}_{t}^{2}\right)(x) L(d s, d x)\right] \\
\leqslant & 4 \gamma \int_{0}^{\delta} S_{t+\varepsilon+\delta-s}\left(\widetilde{X}_{0}^{1}\right)(x) S_{t+\varepsilon+\delta-s}\left(\widetilde{X}_{0}^{2}\right)(x) \\
& \left.\times P\left[S_{s}\left(X_{0}^{1}\right)(x) S_{s}\left(X_{0}^{2}\right)(x)\right] d x d s \quad \text { (by Lemma } 2.2(\mathrm{c})\right) \\
\leqslant & 4 \gamma c_{A 1}(t+1, \lambda)^{2}\left|\tilde{x}_{0}^{1}\right|_{\lambda}\left|\tilde{x}_{0}^{2}\right|_{\lambda} \\
& \times P\left[\int_{\mathbb{R}^{2}} \phi_{2 \lambda}(x) \int_{0}^{\delta} S_{s}\left(X_{0}^{1}\right)(x) S_{s}\left(X_{0}^{2}\right)(x) d s d x\right] \quad \text { (by Lemma A.1) } \\
= & 4 \gamma c_{A 1}(t+1, \lambda)^{2}\left|\tilde{x}_{0}^{1}\right|_{\lambda}\left|\tilde{x}_{0}^{2}\right|_{\lambda} P\left[\int_{\mathbb{R}^{2}} \phi_{2 \lambda}(x) \bar{g}_{\delta}\left(X_{0}^{1}, X_{0}^{2}\right)(x) d x\right] \\
\rightarrow & 0, \quad \text { as } \delta \downarrow 0, \text { uniformly in } \varepsilon,
\end{aligned}
$$

where the last convergence follows by (EnC) and Dominated Convergence. This gives the desired result.

\section{Proof of Theorem 1.8}

We start with the following lemma.

LEMMA 3.1 (Uniqueness of one-dimensional distributions). - Assume $\gamma / \sigma^{2}<$ $\left(\sqrt{6} \pi c_{\mathrm{rw}}\right)^{-1}$ and $\mathbf{X}_{0} \in \mathcal{M}_{\text {tem,e }}$. Let $\mathbf{X}$ and $\mathbf{Z}$ be any two solutions to $(\mathbf{M P})_{\mathbf{X}_{0}}^{\sigma, \gamma}$ satisfying (IntC) with initial conditions satisfying (EnC). Then $\mathbf{X}$ and $\mathbf{Z}$ have the same onedimensional distributions, that is, for each $t>0$

$$
P\left(\mathbf{X}_{t} \in \Gamma\right)=P\left(\mathbf{Z}_{t} \in \Gamma\right), \quad \forall \Gamma \in \mathcal{B}\left(\mathcal{M}_{\text {tem }}^{2}\right) .
$$

Proof. - A monotone class argument shows the bounded pointwise closure of the complex linear span of $\left\{\mathfrak{E}\left(\cdot, \widetilde{\mathbf{X}}_{0}\right): \widetilde{\mathbf{X}}_{0} \in\left(\mathcal{C}_{\text {rap }}^{+}\right)^{2}\right\}$ is the set of all bounded complexvalued measurable maps on $\mathcal{M}_{\text {tem }}^{2}$ (e.g. see Lemma 6.2 of [5]). Therefore, the result is immediate from Proposition 1.12.

Before we give a proof of Theorem 1.8 let us prove two useful lemmas.

Lemma 3.2. - Let $\mathbf{X}$ be a solution to $(\mathbf{M P})_{\mathbf{X}_{0}}^{\sigma, \gamma}$ on $\left(\Omega, \mathcal{F}, \mathcal{F}_{t}, P\right)$ satisfying (IntC) and (EnC). Then 
(a) $\mathbf{X}_{t} \in \mathcal{M}_{\text {tem,e }}$ for any $t \geqslant 0$ P-a.s., and for any bounded stopping time $\tau$

$$
P\left[\int_{\mathbb{R}^{2}} \bar{g}_{\delta}\left(X_{\tau}^{1}, X_{\tau}^{2}\right)(x) \phi_{\lambda}(x) d x\right]<\infty, \quad \forall \delta>0, \forall \lambda>0 .
$$

(b) For any bounded stopping time $\tau$

$$
P\left[\sum_{j=1}^{2} X_{\tau}^{j}\left(\phi_{\lambda}\right)^{2}\right]<\infty, \quad \forall \lambda>0 .
$$

Proof. -

(a) The proof essentially goes along the lines of the proof of Proposition 25 of [3] with changes necessitated by the infinite measure states. Define

$$
\begin{aligned}
& g_{\alpha}\left(\mu_{1}, \mu_{2}\right)(x) \\
& \quad \equiv \int_{0}^{\infty} \mathrm{e}^{-\alpha u} S_{u} \mu_{1}(x) S_{u} \mu_{2}(x) d u, \quad \forall \alpha>0, x \in \mathbb{R}^{2},\left(\mu_{1}, \mu_{2}\right) \in \mathcal{M}_{\text {tem }, \mathrm{e}}, \\
& g_{\alpha, \varepsilon}\left(\mu_{1}, \mu_{2}\right)(x) \\
& \equiv \int_{\varepsilon}^{\infty} \mathrm{e}^{-\alpha u} S_{u} \mu_{1}(x) S_{u} \mu_{2}(x) d u, \quad \forall \alpha>0, x \in \mathbb{R}^{2},\left(\mu_{1}, \mu_{2}\right) \in \mathcal{M}_{\text {tem }, \mathrm{e}} .
\end{aligned}
$$

Fix arbitrary $\lambda>0$. For any $t>0, \bar{g}_{t}\left(\mu_{1}, \mu_{2}\right)(x) \leqslant \mathrm{e}^{\alpha t} g_{\alpha}\left(\mu_{1}, \mu_{2}\right)(x)$. Therefore it is enough to check that

$$
\sup _{t \leqslant T} \int_{\mathbb{R}^{2}} g_{\alpha}\left(X_{t}^{1}, X_{t}^{2}\right)(x) \phi_{\lambda}(x) d x<\infty, \quad P \text {-a.s. }, \forall 0<T<\infty,
$$

and

$$
P\left[\int_{\mathbb{R}^{2}} g_{\alpha}\left(X_{\tau}^{1}, X_{\tau}^{2}\right)(x) \phi_{\lambda}(x) d x\right]<\infty
$$

for any bounded stopping time $\tau$. It follows from Itô's formula, just as in the derivation of $\left(T_{\varepsilon}\right)$ in Section 5 of [1], that

$$
\begin{aligned}
\int_{\mathbb{R}^{2}} g_{\alpha, \varepsilon}\left(X_{t}^{1}, X_{t}^{2}\right)(x) \phi_{\lambda}(x) d x= & \int_{\mathbb{R}^{2}} g_{\alpha, \varepsilon}\left(X_{0}^{1}, X_{0}^{2}\right)(x) \phi_{\lambda}(x) d x \\
& -\int_{0}^{t} \mathrm{e}^{-\alpha \varepsilon} \int_{\mathbb{R}^{2}} \bar{p}_{\varepsilon}\left(X_{s}^{1}, X_{s}^{2}\right)(x) \phi_{\lambda}(x) d x d s \\
& +\alpha \int_{0}^{t} \int_{\mathbb{R}^{2}} g_{\alpha, \varepsilon}\left(X_{s}^{1}, X_{s}^{2}\right)(x) \phi_{\lambda}(x) d x d s+M_{t}^{\varepsilon}
\end{aligned}
$$


where $M_{t}^{\varepsilon}$ is a local martingale. Then

$$
\begin{aligned}
& \mathrm{e}^{-\alpha t} \int_{\mathbb{R}^{2}} g_{\alpha, \varepsilon}\left(X_{t}^{1}, X_{t}^{2}\right)(x) \phi_{\lambda}(x) d x \\
& =\int_{\mathbb{R}^{2}} g_{\alpha, \varepsilon}\left(X_{0}^{1}, X_{0}^{2}\right)(x) \phi_{\lambda}(x) d x \\
& \quad-\int_{0}^{t} \mathrm{e}^{-\alpha(s+\varepsilon)} \int_{\mathbb{R}^{2}} \bar{p}_{\varepsilon}\left(X_{s}^{1}, X_{s}^{2}\right)(x) \phi_{\lambda}(x) d x d s+\int_{0}^{t} \mathrm{e}^{-\alpha s} d M_{s}^{\varepsilon} .
\end{aligned}
$$

This shows that $\mathrm{e}^{-\alpha t} \int_{\mathbb{R}^{2}} g_{\alpha, \varepsilon}\left(X_{t}^{1}, X_{t}^{2}\right)(x) \phi_{\lambda}(x) d x$ is a positive supermartingale. Letting $\varepsilon \downarrow 0$ we get by Fatou lemma that the limiting process $\mathrm{e}^{-\alpha t} \int_{\mathbb{R}^{2}} g_{\alpha}\left(X_{t}^{1}, X_{t}^{2}\right)(x) \phi_{\lambda}(x) d x$ is also a positive supermartingale. This together with maximal inequality for positive supermartingales, Optional Stopping Theorem and (EnC) gives the desired result.

(b) Fix arbitrary $\lambda>0$. We can choose $\tilde{\phi} \geqslant \phi_{\lambda}$ such that $\tilde{\phi} \in \mathcal{C}_{\lambda}^{(2)}$. Since $\tau$ is bounded, it is enough to check that

$$
P\left[\sup _{0 \leqslant t \leqslant T} \sum_{j=1}^{2} X_{t}^{j}(\tilde{\phi})^{2}\right]<\infty, \quad \forall T>0
$$

But this follows by a simple moment calculation combined with Doob's maximal inequality.

LEMMA 3.3. - Let $\mathbf{X}$ be a solution to $(\mathbf{M P})_{\mathbf{X}_{0}^{\sigma, \gamma}}^{\sigma}$ on $\left(\Omega, \mathcal{F}, \mathcal{F}_{t}, P\right)$ satisfying (IntC) and (EnC). Let $\tau$ be any bounded stopping time. Fix arbitrary $F \in \mathcal{F}_{\tau}$ with $P(F)>0$. Define

$$
P_{1}(B)=P\left(1_{F} P\left(1_{B} \mid \mathcal{F}_{\tau}\right)\right) / P(F), \quad B \in \mathcal{F},
$$

$\mathcal{F}_{t}^{\tau} \equiv \mathcal{F}_{\tau+t}$, and $\widehat{\mathbf{X}}_{t} \equiv \mathbf{X}_{\tau+t}$. Then $\widehat{\mathbf{X}}$ solves $(\mathbf{M P})_{\widehat{\mathbf{X}}_{0}}^{\sigma, \gamma}$ on $\left(\Omega, \mathcal{F}, \mathcal{F}_{t}^{\tau}, P_{1}\right)$ with $\mathcal{L}\left(\widehat{\mathbf{X}}_{0}\right)=$ $P\left(\mathbf{X}_{\tau} \in \cdot \mid F\right)$ and satisfies (IntC) and (EnC).

Proof. - Since $\tau$ is bounded there exists $M>0$ such that $\tau \leqslant M, P$-a.s. It is easy to check that $\widehat{\mathbf{X}}$ solves $(\mathbf{M P})_{\widehat{\mathbf{X}}_{0}}^{\sigma, \gamma}$ on $\left(\Omega, \mathcal{F}, \mathcal{F}_{t}^{\tau}, P_{1}\right)$ with $\mathcal{L}\left(\widehat{\mathbf{X}}_{0}\right)=P\left(\mathbf{X}_{\tau} \in \cdot F\right)$. Let us check that it satisfies (IntC) and (EnC). It is enough to check (IntC) and (EnC) for $F=\Omega$. Fix arbitrary $\delta>0$. Take a sequence $F_{1} \subset F_{2} \subset \cdots$ of events in $\mathcal{F}_{\delta}$ such that $F_{l} \uparrow \Omega$, as $l \rightarrow \infty$ and for each $n \geqslant 1$ and $t>\delta$

$$
\underset{\varepsilon \downarrow 0}{\limsup } P\left[\int_{\delta}^{t} H_{\varepsilon, K}\left(\mathbf{X}_{s}\right) d s 1_{F_{n}}\right]<\infty, \quad \forall \text { compact set } K \subset \mathbb{R}^{2}
$$

Then for each $n \geqslant 1, t>\delta$

$$
\underset{\varepsilon \downarrow 0}{\limsup P}\left[\int_{\delta}^{t} H_{\varepsilon, K}\left(\widehat{\mathbf{X}}_{s}\right) d s 1_{F_{n}}\right]=\underset{\varepsilon \downarrow 0}{\limsup } P\left[\int_{\tau+\delta}^{\tau+t} H_{\varepsilon, K}\left(\mathbf{X}_{s}\right) d s 1_{F_{n}}\right]
$$




$$
\begin{aligned}
& \leqslant \underset{\varepsilon \downarrow 0}{\limsup P}\left[\int_{\delta}^{M+t} H_{\varepsilon, K}\left(\mathbf{X}_{s}\right) d s 1_{F_{n}}\right] \\
& <\infty, \quad \forall \text { compact set } K \subset \mathbb{R}^{2},
\end{aligned}
$$

by (3.7). Therefore $\widehat{\mathbf{X}}$ also satisfies (IntC) (note that $\left\{\widehat{\mathbf{X}}_{t}\right\}$ is adapted to $\left\{\mathcal{F}_{\tau+t}\right\}$ ) and the result follows. Regarding (EnC) it follows from Lemma 3.2.

Proof of Theorem 1.8. - We argue as in the proof of Theorem 4.4.2 [7]. Let $\mathbf{X}, \mathbf{Z}$ be any two solutions to (MP) $)_{\mathbf{X}_{0}}^{\sigma, \gamma}$ satisfying (IntC). We want to show that

$$
P\left[\prod_{k=1}^{m} f_{k}\left(\mathbf{X}_{t_{k}}\right)\right]=P\left[\prod_{k=1}^{m} f_{k}\left(\mathbf{Z}_{t_{k}}\right)\right]
$$

for all choices of $t_{k} \in[0, \infty)$ and bounded Borel measurable functions $f_{k}$ on $\mathcal{M}_{\text {tem }}^{2}$. It is sufficient to consider only $f_{k}>0$. For $m=1$ (3.8) follows from Lemma 3.1. Proceeding by induction, assume (3.8) holds for all $m \leqslant n$. Fix $0 \leqslant t_{1}<t_{2} \cdots<t_{n}$ and bounded strictly positive Borel measurable functions $f_{1}, \ldots, f_{n}$ on $\mathcal{M}_{\text {tem }}^{2}$. Define

$$
\begin{array}{ll}
P^{1}(B)=\frac{P\left[1_{B} \prod_{k=1}^{n} f_{k}\left(\mathbf{X}_{t_{k}}\right)\right]}{P\left[\prod_{k=1}^{n} f_{k}\left(\mathbf{X}_{t_{k}}\right)\right]}, & B \in \mathcal{F}^{X}, \\
P^{2}(B)=\frac{P\left[1_{B} \prod_{k=1}^{n} f_{k}\left(\mathbf{Z}_{t_{k}}\right)\right]}{P\left[\prod_{k=1}^{n} f_{k}\left(\mathbf{Z}_{t_{k}}\right)\right]}, & B \in \mathcal{F}^{Z},
\end{array}
$$

and set $\widehat{\mathbf{X}}=\mathbf{X}_{t_{n}+t}, \widehat{\mathbf{Z}}=\mathbf{Z}_{t_{n}+t}$. Then Lemma 3.3 shows that $\widehat{\mathbf{X}}$ and $\widehat{\mathbf{Z}}$ solve $(\mathbf{M P})_{v}^{\sigma, \gamma}$ on $\left(\Omega, \mathcal{F}, \mathcal{F}_{t}^{t_{n}}, P^{1}\right)$ and $\left(\Omega, \mathcal{F}, \mathcal{F}_{t}^{t_{n}}, P^{2}\right)$, respectively, with the same initial distribution $v$ (the latter by the induction assumption). $\widehat{\mathbf{X}}$ and $\widehat{\mathbf{Z}}$ also satisfy (IntC) and (EnC) by Lemma 3.3, and therefore by Lemma 3.1 they have the same one dimensionaldistributions. This implies that for any bounded Borel measurable function $f$ on $\mathcal{M}_{\text {tem }}^{2}$ we have

$$
P^{1}\left[f\left(\widehat{\mathbf{X}}_{t}\right)\right]=P^{2}\left[f\left(\widehat{\mathbf{Z}}_{t}\right)\right], \quad \forall t \geqslant 0 .
$$

It follows from the definitions of $P^{1}$ and $P^{2}$ and the induction hypothesis that

$$
P\left[f\left(\mathbf{X}_{t_{n}+t}\right) \prod_{k=1}^{n} f_{k}\left(\mathbf{X}_{t_{k}}\right)\right]=P\left[f\left(\mathbf{Z}_{t_{n}+t}\right) \prod_{k=1}^{n} f_{k}\left(\mathbf{Z}_{t_{k}}\right)\right],
$$

and by setting $t_{n+1}=t_{n}+t$ we get (3.8) for $m=n+1$.

Proof of Theorem 1.11. - As it has been mentioned already in Remark 2.12 the proof of Theorem 1.11(a) was completed by Corollary 2.11. Part (b) of Theorem 1.11 follows now from Theorem 1.8 and Theorem 1.11(a). 


\section{Continuity of the transition function with respect to initial conditions}

For any $\lambda, t>0$ let $c_{A 3}(t, \lambda), c_{A 4}(t)$ be the constants defined in Lemma A.3. To simplify the notation define also the constants

$$
\begin{aligned}
& c_{4.1}(\mu, \lambda, t, \delta) \equiv 4 \gamma c_{A 3}(t, \lambda)^{2} c_{A 4}(t-\delta)^{2} \mu^{1}\left(\phi_{\lambda}\right) \mu^{2}\left(\phi_{\lambda}\right), \\
& \forall \mu=\left(\mu^{1}, \mu^{2}\right) \in \mathcal{M}_{\text {tem }, \mathrm{e}}, 0<\delta<t, \lambda>0, \\
& c_{4.2}(\mu, \lambda, t, \delta) \equiv 4 \gamma c_{A 3}(t, \lambda)^{2} c_{A 4}(t-\delta)^{2} \mu^{1}\left(\phi_{-\lambda}\right) \mu^{2}\left(\phi_{-\lambda}\right), \\
& \forall \mu=\left(\mu^{1}, \mu^{2}\right) \in \mathcal{M}_{\text {rap }, \mathrm{e}}, 0<\delta<t, \lambda>0 .
\end{aligned}
$$

LEMMA 4.1. -

(a) Let $\mu=\left(\mu^{1}, \mu^{2}\right) \in \mathcal{M}_{\text {tem,e, }} \tilde{\mu}=\left(\tilde{\mu}^{1}, \tilde{\mu}^{2}\right) \in \mathcal{M}_{\text {rap,e. }}$ Let $\mathbf{X}$ solve the martingale problem (MP) ${ }_{\mu}^{\sigma, \gamma}$. Then for all $0<\delta<t, \lambda>0$

$$
\begin{aligned}
& \underset{\varepsilon \downarrow 0}{\limsup }\left|P\left[\mathfrak{E}\left(S_{\varepsilon} \mathbf{X}_{t}, \tilde{\mu}\right)\right]-P\left[\mathfrak{E}\left(S_{\delta} \mathbf{X}_{t-\delta}, \tilde{\mu}\right)\right]\right| \\
& \quad \leqslant c_{4.1}(\mu, \lambda, t, \delta) \int_{\mathbb{R}^{2}} \bar{g}_{\delta}\left(\tilde{\mu}^{1}, \tilde{\mu}^{2}\right)(x) \phi_{-2 \lambda}(x) d x<\infty .
\end{aligned}
$$

(b) Let $x_{0}=\left(x_{0}^{1}, x_{0}^{2}\right) \in \mathcal{B}_{\mathrm{b}}^{+} \times \mathcal{B}_{\mathrm{b}}^{+}, \tilde{\mu}=\left(\tilde{\mu}^{1}, \tilde{\mu}^{2}\right) \in \mathcal{M}_{\mathrm{f}, \mathrm{e}}$. Let $\mathbf{X}$ solve the martingale problem (MP) $)_{x_{0}}^{\sigma, \gamma}$. Then for all $0<\delta<t$,

$$
\begin{aligned}
& \underset{\varepsilon \downarrow 0}{\limsup }\left|P\left[\mathfrak{E}\left(S_{\varepsilon} \mathbf{X}_{t}, \tilde{\mu}\right)\right]-P\left[\mathfrak{E}\left(S_{\delta} \mathbf{X}_{t-\delta}, \tilde{\mu}\right)\right]\right| \\
& \quad \leqslant 4 \gamma\left|x_{0}^{1}\right|_{\infty}\left|x_{0}^{2}\right|_{\infty} \int_{\mathbb{R}^{2}} \bar{g}_{\delta}\left(\tilde{\mu}^{1}, \tilde{\mu}^{2}\right)(x) d x<\infty .
\end{aligned}
$$

(c) Let $\mu=\left(\mu^{1}, \mu^{2}\right) \in \mathcal{M}_{\text {rap,e },}, \tilde{\mu}=\left(\tilde{\mu}^{1}, \tilde{\mu}^{2}\right) \in \mathcal{M}_{\text {tem,e }}$. Let $\mathbf{X}$ solve the martingale problem (MP) ${ }_{\mu}^{\sigma, \gamma}$. Then for all $0<\delta<t, \lambda>0$

$$
\begin{aligned}
& \underset{\varepsilon \downarrow 0}{\limsup }\left|P\left[\mathfrak{E}\left(S_{\varepsilon} \mathbf{X}_{t}, \tilde{\mu}\right)\right]-P\left[\mathfrak{E}\left(S_{\delta} \mathbf{X}_{t-\delta}, \tilde{\mu}\right)\right]\right| \\
& \quad \leqslant c_{4.2}(\mu, \lambda, t, \delta) \int_{\mathbb{R}^{2}} \bar{g}_{\delta}\left(\tilde{\mu}^{1}, \tilde{\mu}^{2}\right)(x) \phi_{2 \lambda}(x) d x<\infty .
\end{aligned}
$$

Proof. - (a) By Lemma 2.3(b) we get that for any $\varepsilon>0$,

$$
\begin{aligned}
& \left|P\left[\mathfrak{E}\left(S_{\varepsilon} \mathbf{X}_{t}, \tilde{\mu}\right)\right]-P\left[\mathfrak{E}\left(S_{\delta} \mathbf{X}_{t-\delta}, \tilde{\mu}\right)\right]\right| \\
& \leqslant\left|4 \gamma P\left[\int_{t-\delta}^{t} \mathfrak{E}\left(S_{\varepsilon+t-s} \mathbf{X}_{s}, \tilde{\mu}\right) \int_{\mathbb{R}^{2}} S_{\varepsilon+t-s} \tilde{\mu}^{1}(x) S_{\varepsilon+t-s} \tilde{\mu}^{2}(x) L(d s, d x)\right]\right| \\
& \quad+\left|P\left[\mathfrak{E}\left(S_{\varepsilon+\delta} \mathbf{X}_{t-\delta}, \tilde{\mu}\right)\right]-P\left[\mathfrak{E}\left(S_{\delta} \mathbf{X}_{t-\delta}, \tilde{\mu}\right)\right]\right| .
\end{aligned}
$$

The first term in (4.1) is bounded by

$$
4 \gamma P\left[\int_{t-\delta}^{t} \int_{\mathbb{R}^{2}} S_{\varepsilon+t-s} \tilde{\mu}^{1}(x) S_{\varepsilon+t-s} \tilde{\mu}^{2}(x) L(d s, d x)\right]
$$




$$
\begin{aligned}
\leqslant & 4 \gamma \int_{t-\delta}^{t} \int_{\mathbb{R}^{2}} S_{\varepsilon+t-s} \tilde{\mu}^{1}(x) S_{\varepsilon+t-s} \tilde{\mu}^{2}(x) S_{s} \mu^{1}(x) S_{s} \mu^{2}(x) d s d x \\
\leqslant & 4 \gamma \int_{0}^{\varepsilon+\delta} \int_{\mathbb{R}^{2}} c_{A 3}(t, \lambda)^{2} c_{A 4}(t-\delta)^{2} \phi_{-2 \lambda}(x) \mu^{1}\left(\phi_{\lambda}\right) \\
& \times \mu^{2}\left(\phi_{\lambda}\right) 1(\varepsilon \leqslant s \leqslant \varepsilon+\delta) S_{s} \tilde{\mu}^{1}(x) S_{s} \tilde{\mu}^{2}(x) d s d x \\
= & c_{4.1}(\mu, \lambda, t, \delta) \int_{0}^{\varepsilon+\delta} \int_{\mathbb{R}^{2}} 1(\varepsilon \leqslant s \leqslant \varepsilon+\delta) S_{s} \tilde{\mu}^{1}(x) S_{s} \tilde{\mu}^{2}(x) \phi_{-2 \lambda}(x) d s d x,
\end{aligned}
$$

where the second inequality follows from Corollary A.4. Now let $\varepsilon \downarrow 0$. By Corollaries A.4, A.5 and Dominated Convergence we get that the second term in (4.1) converges to 0 . Applying again Dominated Convergence we get

$$
\begin{aligned}
& \lim _{\varepsilon \downarrow 0} \int_{0}^{\varepsilon+\delta} \int_{\mathbb{R}^{2}} 1(\varepsilon \leqslant s \leqslant \varepsilon+\delta) S_{s} \tilde{\mu}^{1}(x) S_{s} \tilde{\mu}^{2}(x) \phi_{-2 \lambda}(x) d s d x \\
& \quad=\int_{\mathbb{R}^{2}} \bar{g}_{\delta}\left(\tilde{\mu}^{1}, \tilde{\mu}^{2}\right)(x) \phi_{-2 \lambda}(x) d x<\infty,
\end{aligned}
$$

and the result follows.

The proof of (b), (c) is completely analogous and therefore is omitted.

PROPOSITION 4.2 (Continuity of transition function with respect to initial conditions). Let $\mu_{n} \rightarrow \mu$ in $\mathcal{M}_{\text {tem,e }}$, such that

$$
\lim _{\delta \downarrow 0} \sup _{n} \int_{\mathbb{R}^{2}} \bar{g}_{\delta}\left(\mu_{n}^{1}, \mu_{n}^{2}\right)(x) \phi_{\lambda}(x) d x=0, \quad \forall \lambda>0 .
$$

Assume that there exist solutions $\mathbf{X}^{n}, \mathbf{X}$ to the martingale problems $(\mathbf{M P})_{\mu_{n}}^{\sigma, \gamma}$ and (MP) ${ }_{\mu}^{\sigma, \gamma}$ respectively, satisfying (IntC), and let $\mathrm{P}_{t}\left(\mu_{n}, \cdot\right)$ and $\mathrm{P}_{t}(\mu, \cdot)$ be their onedimensional distributions on $\mathcal{M}_{\mathrm{tem}}^{2}$. Then

$$
\mathrm{P}_{t}\left(\mu_{n}, \cdot\right) \rightarrow \mathrm{P}_{t}(\mu, \cdot), \quad \forall t \geqslant 0
$$

as $n \rightarrow \infty$.

Proof. - To prove the proposition we have to check weak convergence

$$
\mathbf{X}_{t}^{n} \Rightarrow \mathbf{X}_{t} \quad \text { in } \mathcal{M}_{\mathrm{tem}}^{2}, \text { as } n \rightarrow \infty, \forall t>0 .
$$

To this end it is enough to check that for arbitrary $\varphi=\left(\varphi^{1}, \varphi^{2}\right) \in\left(\mathcal{C}_{\text {rap }}\right)^{2}$

$$
\left(X_{t}^{1, n}\left(\varphi^{1}\right), X_{t}^{2, n}\left(\varphi^{2}\right)\right) \Rightarrow\left(X_{t}^{1}\left(\varphi^{1}\right), X_{t}^{2}\left(\varphi^{2}\right)\right), \quad \text { as } n \rightarrow \infty, \forall t>0,
$$


and

$$
\sup _{n} P\left[X_{t}^{j, n}\left(\phi_{\lambda}\right)\right]<\infty, \quad j=1,2, \forall t>0, \forall \lambda>0 .
$$

(4.4) follows immediately from Lemma 2.2(c), Lemma A.1 and our assumptions on the initial measures $\mu_{n}^{1}, \mu_{n}^{2}$.

To check (4.3), fix arbitrary $\varphi=\left(\varphi^{1}, \varphi^{2}\right) \in\left(\mathcal{C}_{\text {rap }}^{+}\right)^{2}$ and let $\widetilde{\mathbf{X}}$ be a solution to the martingale problem $(\mathbf{M P})_{\varphi}^{\sigma, \gamma}$. Then

$$
\begin{aligned}
\mid P[ & \left.\mathfrak{E}\left(\mathbf{X}_{t}^{n}, \varphi\right)\right]-P\left[\mathfrak{E}\left(\mathbf{X}_{t}, \varphi\right)\right] \mid \\
= & \left|\lim _{\varepsilon \downarrow 0} \widetilde{P}\left[\mathfrak{E}\left(\mu_{n}, S_{\varepsilon} \widetilde{\mathbf{X}}_{t}\right)\right]-\lim _{\varepsilon \downarrow 0} \widetilde{P}\left[\mathfrak{E}\left(\mu, S_{\varepsilon} \widetilde{\mathbf{X}}_{t}\right)\right]\right|, \quad \text { (by Proposition 1.12) } \\
\leqslant & \left|\lim _{\varepsilon \downarrow 0} \widetilde{P}\left[\mathfrak{E}\left(\mu_{n}, S_{\varepsilon} \widetilde{\mathbf{X}}_{t}\right)\right]-\widetilde{P}\left[\mathfrak{E}\left(\mu_{n}, S_{\delta} \widetilde{\mathbf{X}}_{t-\delta}\right)\right]\right| \\
& +\left|\widetilde{P}\left[\mathfrak{E}\left(\mu_{n}, S_{\delta} \widetilde{\mathbf{X}}_{t-\delta}\right)\right]-\widetilde{P}\left[\mathfrak{E}\left(\mu, S_{\delta} \widetilde{\mathbf{X}}_{t-\delta}\right)\right]\right| \\
& +\left|\widetilde{P}\left[\mathfrak{E}\left(\mu, S_{\delta} \widetilde{\mathbf{X}}_{t-\delta}\right)\right]-\lim _{\varepsilon \downarrow 0} \widetilde{P}\left[\mathfrak{E}\left(\mu, S_{\varepsilon} \widetilde{\mathbf{X}}_{t}\right)\right]\right| \\
\leqslant & \left.c_{4.2}(\varphi, \lambda, t, \delta) \int_{\mathbb{R}^{2}}\left(\bar{g}_{\delta}\left(\mu_{n}^{1}, \mu_{n}^{2}\right)(x)+\bar{g}_{\delta}\left(\mu^{1}, \mu^{2}\right)(x)\right) \phi_{\lambda}(x) d x \quad \text { (by Lemma 4.1(c) }\right) \\
& +\left|\widetilde{P}\left[\mathfrak{E}\left(\mu_{n}, S_{\delta} \widetilde{\mathbf{X}}_{t-\delta}\right)\right]-\widetilde{P}\left[\mathfrak{E}\left(\mu, S_{\delta} \widetilde{\mathbf{X}}_{t-\delta}\right)\right]\right|, \quad \forall t>0, n \geqslant 1 .
\end{aligned}
$$

Let $n \rightarrow \infty$. Then by Lemmas 2.4 and A.1,

$$
\lim _{n \rightarrow \infty}\left|\widetilde{P}\left[\mathfrak{E}\left(\mu_{n}, S_{\delta} \widetilde{\mathbf{X}}_{t-\delta}\right)\right]-\widetilde{P}\left[\mathfrak{E}\left(\mu, S_{\delta} \widetilde{\mathbf{X}}_{t-\delta}\right)\right]\right|=0 .
$$

Therefore

$$
\begin{gathered}
\limsup _{n \rightarrow \infty}\left|P\left[\mathfrak{E}\left(\mathbf{X}_{t}^{n}, \varphi\right)\right]-P\left[\mathfrak{E}\left(\mathbf{X}_{t}, \varphi\right)\right]\right| \leqslant \\
\sup _{n} c_{4.2}(\varphi, \lambda, t, \delta) \int_{\mathbb{R}^{2}}\left(\bar{g}_{\delta}\left(\mu_{n}^{1}, \mu_{n}^{2}\right)(x)\right. \\
\left.+\bar{g}_{\delta}\left(\mu^{1}, \mu^{2}\right)(x)\right) \phi_{\lambda}(x) d x
\end{gathered}
$$

for any $\delta>0$. Let $\delta \downarrow 0$. Then it follows from our assumptions on $\mu_{n}^{1}, \mu_{n}^{2}$ that

$$
\limsup _{n \rightarrow \infty}\left|P\left[\mathfrak{E}\left(\mathbf{X}_{t}^{n}, \varphi\right)\right]-P\left[\mathfrak{E}\left(\mathbf{X}_{t}, \varphi\right)\right]\right|=0, \quad \forall t \geqslant 0 .
$$

By a standard argument, the last convergence implies (4.3) and we are done.

\section{Finite initial conditions}

\subsection{Existence of a process satisfying (IntC) and proof of Theorem 1.9(a),(b)}

We recall some facts about the dual process $\left(\phi_{t}, I_{t}\right)$ introduced prior to Theorem 32 of [3]. This process takes values in $\mathcal{S}=\mathcal{M}_{\mathrm{f}}\left(\left(\mathbb{R}^{2}\right)^{4}\right) \times 2^{\{1, \ldots, 4\}}$ and points in $\mathcal{S}$ are denoted by $(\phi, I)$. For $1 \leqslant j, j^{\prime} \leqslant 4$ define maps $\pi_{j, j^{\prime}}:\left(\mathbb{R}^{2}\right)^{4} \mapsto\left(\mathbb{R}^{2}\right)^{4}$ and $f_{j, j^{\prime}}: \mathcal{C}_{\mathrm{b}}^{+}\left(\left(\mathbb{R}^{2}\right)^{4}\right) \mapsto$ $\mathcal{M}_{\mathrm{f}}\left(\left(\mathbb{R}^{2}\right)^{4}\right)$ by 


$$
\begin{gathered}
\left(\pi_{j, j^{\prime}} x\right)_{i}=\left\{\begin{array}{ll}
x_{i}, & \text { if } i \neq j^{\prime}, \\
x_{j}, & \text { if } i=j^{\prime},
\end{array} \quad x=\left(x_{1}, x_{2}, x_{3}, x_{4}\right),\right. \\
f_{j, j^{\prime}}(\phi)(A)=\int_{A} \phi\left(\pi_{j, j^{\prime}} x\right) \delta_{0}\left(x_{j}-x_{j^{\prime}}\right) d x_{1} d x_{2} d x_{3} d x_{4} .
\end{gathered}
$$

Let $\vec{S}_{t}$ be the 8-dimensional Brownian semigroup with variance $\sigma^{2}$. The dynamics of the dual process, $(\phi ., I.) \in D\left(\mathbb{R}_{+}, \mathcal{S}\right)$ are as follows:

(a) For each $\left(j, j^{\prime}\right) \in I_{t} \times I_{t}, j \neq j^{\prime}$, with rate $\gamma / 2,\left(\phi_{t-}, I_{t-}\right)$ jumps to $\left(f_{j, j^{\prime}}\left(\phi_{t-}\right)\right.$, $\left.I_{t-}-\left\{j^{\prime}\right\}\right)$, and for each $\left(j, j^{\prime}\right) \in I_{t}^{c} \times I_{t}^{c}, j \neq j^{\prime}$, with rate $\gamma / 2,\left(\phi_{t-}, I_{t-}\right)$ jumps to $\left(f_{j, j^{\prime}}\left(\phi_{t-}\right), I_{t-} \cup\left\{j^{\prime}\right\}\right)$. In either case we will say $j^{\prime}$ switches via $j$.

(b) Let $0=T_{0}<T_{1}<T_{2}<\cdots$ be the successive jump times. Then for $T_{n} \leqslant t<T_{n+1}$, $\left(\phi_{t}, I_{t}\right)=\left(\vec{S}_{t-T_{n}} \phi_{T_{n}}, I_{T_{n}}\right)$.

Let $\widehat{P}_{\phi_{0}, I_{0}}$ be the law of $\left(\phi ., I\right.$.) on $D\left(\mathbb{R}_{+}, \mathcal{S}\right)$. Note again that we identify functions $\phi$ in $\mathcal{C}_{\mathrm{b}}\left(\mathbb{R}^{8}\right) \cap L^{1}(d x)$ with $\phi(x) d x \in \mathcal{M}_{\mathrm{f}}\left(\mathbb{R}^{8}\right)$. Let $U_{n}=T_{n}-T_{n-1}, n \geqslant 1$. Then under $\widehat{P}_{\phi_{0}, I_{0}}$

$\left\{U_{n}\right\}$ are independent exponential random variables and

$$
\text { if }\left|I_{0}\right|=2, U_{2 n+1} \text { has mean }(2 \gamma)^{-1} \text { while } U_{2 n} \text { has mean }(3 \gamma)^{-1} \text {. }
$$

Assume that $\mathbf{X}_{0} \in \mathcal{M}_{\mathrm{f}, \mathrm{e}}$ and $\mathbf{X}$ satisfies martingale problem $(\mathbf{M P})_{\mathbf{X}_{0}}^{\sigma, \gamma}$. We introduce a fourth moment condition on $\mathbf{X}$ : for $\delta \geqslant 0$

(MB) ${ }_{\delta} \forall \phi_{0} \in \mathcal{C}_{\mathrm{b}}^{+}\left(\mathbb{R}^{2}\right)^{4}, I_{0} \subset\{1,2,3,4\}$ and any Borel map $\psi: \mathcal{M}_{\mathrm{f}}\left(\mathbb{R}^{2}\right)^{4} \mapsto \mathbb{R}_{+}, \forall t>\delta$,

$$
\begin{aligned}
& P\left[\int \phi_{0}\left(x_{1}, x_{2}, x_{3}, x_{4}\right) \prod_{i \in I_{0}} X_{t}^{1}\left(d x_{i}\right) \prod_{j \in I_{0}^{c}} X_{t}^{2}\left(d x_{j}\right) \psi\left(\mathbf{X}_{\delta}\right)\right] \\
& \leqslant \widehat{P}_{\phi_{0}, I_{0}} \times P\left[\int \phi_{t-\delta}\left(x_{1}, x_{2}, x_{3}, x_{4}\right) \prod_{i \in I_{t-\delta}} X_{\delta}^{1}\left(d x_{i}\right) \prod_{j \in I_{t-\delta}^{c}} X_{\delta}^{2}\left(d x_{j}\right) \psi\left(\mathbf{X}_{\delta}\right)\right. \\
& \left.\quad \times \exp \left\{\gamma \int_{0}^{t-\delta}\left(\begin{array}{c}
\left|I_{s}\right| \\
2
\end{array}\right)+\left(\begin{array}{c}
\left|I_{s}^{c}\right| \\
2
\end{array}\right) d s\right\}\right] .
\end{aligned}
$$

The existence of a solution satisfying $(\mathrm{MB})_{\delta}$ for any $\delta>0$ was established in Proposition 52 and Theorem 53 of [3] providing

$$
\gamma \sigma^{-2}<\left(3 \sqrt{6} \pi c_{\mathrm{rW}}\right)^{-1} .
$$

In addition conditions were given under which the upper bound is finite (see Theorems 53,54 of [3]).

The following theorem completes the proof of Theorem 1.9(a), by establishing existence of solution satisfying (IntC).

THEOREM 5.1. - Assume

$$
\gamma \sigma^{-2}<6^{-1 / 2} .
$$


(a) If (MB) $\delta$ holds for all $\delta>0$, then $\mathbf{X}$ satisfies (IntC).

(b) If (MB) $)_{0}$ holds and $\mathbf{X}_{0} \in \mathcal{M}_{\mathrm{f}, \mathrm{se}}$, then $\mathbf{X}$ satisfies (SIntC).

The rest of this subsection is devoted to the proof of the above theorem and we will use the following notation.

$$
\begin{gathered}
c_{0}=c_{0}(\sigma)=\left(2 \pi \sigma^{2}\right)^{-1}, \\
c_{1}=c_{1}(\sigma)=2 c_{0}(\sigma)=\left(\pi \sigma^{2}\right)^{-1}, \\
\rho_{n}=c_{1}(\sigma)^{n-1} \prod_{k=1}^{n-1}\left(U_{k}+U_{k+1}\right)^{-1} .
\end{gathered}
$$

For the proof of Theorem 5.1 we will need the following lemma.

LEMMA 5.2. - Assume $I_{0}=\{1,3\}$ and

$$
0 \leqslant \phi_{t}\left(y_{1}, y_{2}, y_{3}, y_{4}\right) \leqslant f(t) \mathrm{p}_{a+b t}\left(y_{1}-y_{2}\right) \mathrm{p}_{c+d t}\left(y_{3}-y_{4}\right), \quad \text { for } 0 \leqslant t<T_{1},
$$

for some $a, c \geqslant 0, b, d \geqslant 1$, and continuous function $f: \mathbb{R}_{+} \mapsto \mathbb{R}_{+}$which is bounded on compacts. Let

$$
\rho(t)= \begin{cases}f(t), & \text { if } T_{0} \leqslant t \leqslant T_{1}, \\ f\left(U_{1}\right)\left(\prod_{k=1}^{n-1} \frac{c_{1}}{\left(U_{k}+U_{k+1}\right)}\right) \frac{c_{1}}{U_{n}+\left(t-T_{n}\right)}, & \text { if } T_{n} \leqslant t<T_{n+1}, n=1,2, \ldots\end{cases}
$$

Then for all $n \geqslant 0$ there are random variables $V_{n}^{i} \geqslant 0, b_{n}^{i} \geqslant 1(i=1,2)$ and random indices $\left\{i_{1}^{n}, \ldots, i_{4}^{n}\right\}=\{1, \ldots, 4\}$ such that

$$
\begin{aligned}
(I)_{n}(a) \quad \phi_{t}(y) \leqslant & \rho(t) \mathrm{p}_{V_{2 n}^{1}+b_{2 n}^{1}\left(t-T_{2 n}\right)}\left(y_{i_{1}^{2 n}}-y_{i_{2}^{2 n}}\right) \\
& \times \mathrm{p}_{V_{2 n}^{2}+b_{2 n}^{2}\left(t-T_{2 n}\right)}\left(y_{i_{3}^{2 n}}-y_{i_{4}^{2 n}}\right), \quad \text { if } T_{2 n}<t<T_{2 n+1}, I_{t}=\left\{i_{1}^{2 n}, i_{3}^{2 n}\right\} \\
(I)_{n}(b) \quad \phi_{t}(y) \leqslant & 2 \rho(t) \mathrm{p}_{V_{2 n+1}^{1}+b_{2 n+1}^{1}\left(t-T_{2 n+1}\right)}\left(y_{i_{1}^{2 n+1}}-y_{i_{2}^{2 n+1}}\right) \\
& \times \mathrm{p}_{2\left(t-T_{2 n+1}\right)}\left(y_{i_{3}^{2 n+1}}-y_{i_{4}^{2 n+1}}\right), \quad \text { if } T_{2 n+1}<t<T_{2(n+1)}, \\
& I_{t}=\left\{i_{3}^{2 n+1}\right\} \text { or }\left\{i_{3}^{2 n+1}\right\}^{c} .
\end{aligned}
$$

Proof. - We proceed by induction on $n$. Note that $(I)_{0}(a)$ holds by assumption with $V_{0}^{1}=a, V_{0}^{2}=c, b_{0}^{1}=b, b_{0}^{2}=d$. Assume that $(I)_{n-1}(a)$ holds for some $n \geqslant 1$. Then, writing $i_{j}$ for $i_{j}^{2(n-1)}$, we have

$$
\begin{aligned}
\phi_{T_{2 n-1}-}(y) \leqslant & f\left(U_{1}\right) \rho_{2 n-1} \mathrm{p}_{V_{2(n-1)}^{1}+b_{2(n-1)}^{1} U_{2 n-1}}\left(y_{i_{1}}-y_{i_{2}}\right) \\
& \times \mathrm{p}_{V_{2(n-1)}^{2}+b_{2(n-1)}^{2} U_{2 n-1}}\left(y_{i_{3}}-y_{i_{4}}\right), \text { and } I_{T_{2 n-1}}=\left\{i_{1}, i_{3}\right\} .
\end{aligned}
$$

By symmetry we may assume $I_{T_{2 n-1}}=\left\{i_{1}\right\}$, i.e. $i_{3}$ switches via $i_{1}$ at $t=T_{2 n-1}$. This gives

$$
\begin{aligned}
\phi_{T_{2 n-1}}(y) \leqslant & f\left(U_{1}\right) \rho_{2 n-1} \mathrm{p}_{V_{2(n-1)}^{1}+b_{2(n-1)}^{1} U_{2 n-1}}\left(y_{i_{1}}-y_{i_{2}}\right) \\
& \times \mathrm{p}_{V_{2(n-1)}^{2}+b_{2(n-1)}^{2} U_{2 n-1}}\left(y_{i_{1}}-y_{i_{4}}\right) \delta_{0}\left(y_{i_{1}}-y_{i_{3}}\right) .
\end{aligned}
$$

Set $W_{2(n-1)}^{i}=V_{2(n-1)}^{i}+b_{2(n-1)}^{i} U_{2 n-1}$ and use the semigroup property of the Brownian densities to see that for $T_{2 n-1}<t<T_{2 n}$, 


$$
\begin{aligned}
\phi_{t}(y) \leqslant & f\left(U_{1}\right) \rho_{2 n-1} \int_{\mathbb{R}^{2}} \mathrm{p}_{W_{2 n-1}^{1}+t-T_{2 n-1}}\left(z_{i_{1}}-y_{i_{2}}\right) \\
& \times \mathrm{p}_{W_{2 n-1}^{2}+t-T_{2 n-1}}\left(z_{i_{1}}-y_{i_{4}}\right) \mathrm{p}_{t-T_{2 n-1}}\left(z_{i_{1}}-y_{i_{3}}\right) \mathrm{p}_{t-T_{2 n-1}}\left(z_{i_{1}}-y_{i_{1}}\right) d z_{i_{1}} \\
\leqslant & f\left(U_{1}\right) \rho_{2 n-1} \int_{\mathbb{R}^{2}}\left[1\left(\left|z_{i_{1}}-y_{i_{2}}\right|<\left|z_{i_{1}}-y_{i_{4}}\right|\right) \mathrm{p}_{W_{2 n-1}^{1}+t-T_{2 n-1}}(0)\right. \\
& \times \mathrm{p}_{W_{2 n-1}^{2}+t-T_{2 n-1}}\left(\frac{y_{i_{2}}-y_{i_{4}}}{2}\right) \\
& \left.+1\left(\left|z_{i_{1}}-y_{i_{2}}\right|>\left|z_{i_{1}}-y_{i_{4}}\right|\right) \mathrm{p}_{W_{2 n-1}^{2}+t-T_{2 n-1}}(0) \mathrm{p}_{W_{2 n-1}^{1}+t-T_{2 n-1}}\left(\frac{y_{i_{2}}-y_{i_{4}}}{2}\right)\right] \\
& \times \mathrm{p}_{t-T_{2 n-1}}\left(z_{i_{1}}-y_{i_{3}}\right) \mathrm{p}_{t-T_{2 n-1}}\left(z_{i_{1}}-y_{i_{1}}\right) d z_{i_{1}} .
\end{aligned}
$$

In the last line we use the fact that $\left|z_{i_{1}}-y_{i_{2}}\right|<\left|z_{i_{1}}-y_{i_{4}}\right|$ implies $\left|z_{i_{1}}-y_{i_{4}}\right| \geqslant\left|y_{i_{2}}-y_{i_{4}}\right| / 2$ and also monotonicity in $|z|$ of $\mathrm{p}_{s}(z)$. Now set $W_{2 n-1}^{m}=W_{2 n-1}^{1} \wedge W_{2 n-1}^{2}$ and $W_{2 n-1}^{M}=$ $W_{2 n-1}^{1} \vee W_{2 n-1}^{2}$. Use the inequality

$$
\mathrm{p}_{t_{2}}(0) \mathrm{p}_{t_{1}}(x) \leqslant \mathrm{p}_{t_{1}}(0) \mathrm{p}_{t_{2}}(x), \quad \text { if } t_{1} \leqslant t_{2},
$$

in (5.6) to conclude that for $T_{2 n-1}<t<T_{2 n}$,

$$
\begin{aligned}
\phi_{t}(y) & \leqslant f\left(U_{1}\right) \rho_{2 n-1} \mathrm{p}_{W_{2 n-1}^{m}+t-T_{2 n-1}}(0) \mathrm{p}_{W_{2 n-1}^{M}+t-T_{2 n-1}}\left(\left(y_{i_{2}}-y_{i_{4}}\right) / 2\right) \mathrm{p}_{2\left(t-T_{2 n-1}\right)}\left(y_{i_{1}}-y_{i_{3}}\right) \\
& \leqslant f\left(U_{1}\right) \rho_{2 n-1} \frac{4 c_{0}}{W_{2 n-1}^{m}+t-T_{2 n-1}} \mathrm{p}_{4 W_{2 n-1}^{M}+4\left(t-T_{2 n-1}\right)}\left(y_{i_{2}}-y_{i_{4}}\right) \mathrm{p}_{2\left(t-T_{2 n-1}\right)}\left(y_{i_{1}}-y_{i_{3}}\right) \\
& \leqslant 2 \rho(t) \mathrm{p}_{4 W_{2 n-1}^{M}+4\left(t-T_{2 n-1}\right)}\left(y_{i_{2}}-y_{i_{4}}\right) \mathrm{p}_{2\left(t-T_{2 n-1}\right)}\left(y_{i_{1}}-y_{i_{3}}\right) .
\end{aligned}
$$

This implies $(I)_{n-1}(b)$ for appropriately chosen $V_{2 n-1}^{1}, i_{j}^{2 n-1}$ and $b_{2 n-1}^{1}=4$. Rather than using this to derive $(I)_{n}(a)$, we can do a bit better with (5.5) which implies (set $\left.W_{2 n}^{i}=W_{2 n-1}^{i}+U_{2 n} \geqslant U_{2 n-1}+U_{2 n}\right)$

$$
\begin{aligned}
\phi_{T_{2 n}}(y) \leqslant & f\left(U_{1}\right) \rho_{2 n-1} \int_{\mathbb{R}^{2}} \mathrm{p}_{W_{2 n}^{1}}\left(z_{i_{1}}-y_{i_{2}}\right) \mathrm{p}_{W_{2 n}^{2}}\left(z_{i_{1}}-y_{i_{4}}\right) \mathrm{p}_{U_{2 n}}\left(z_{i_{1}}-y_{i_{3}}\right) \\
& \times \mathrm{p}_{U_{2 n}}\left(z_{i_{1}}-y_{i_{1}}\right) d z_{i_{1}}, \\
I_{T_{2 n}-}= & \left\{i_{1}\right\} .
\end{aligned}
$$

Note the roles of $i_{2}, i_{4}$ and of $i_{1}, i_{3}$ are symmetric in the above and so there are 3 cases to consider.

Case 1. $i_{4}$ switches via $i_{2}\left(i_{2}\right.$ switches via $i_{4}$ is similar). Then $I_{T_{2 n}}=\left\{i_{1}, i_{4}\right\}$ and

$$
\begin{aligned}
\phi_{T_{2 n}}(y) \leqslant & f\left(U_{1}\right) \rho_{2 n-1} \int_{\mathbb{R}^{2}} \mathrm{p}_{W_{2 n}^{1}}\left(z_{i_{1}}-y_{i_{2}}\right) \mathrm{p}_{W_{2 n}^{2}}\left(z_{i_{1}}-y_{i_{2}}\right) \mathrm{p}_{U_{2 n}}\left(z_{i_{1}}-y_{i_{3}}\right) \\
& \times \mathrm{p}_{U_{2 n}}\left(z_{i_{1}}-y_{i_{1}}\right) d z_{i_{1}} \delta_{0}\left(y_{i_{4}}-y_{i_{2}}\right) .
\end{aligned}
$$

Case 2. $i_{4}$ switches via $i_{3}\left(i_{2}\right.$ switches via $i_{3}$ is similar). Then $I_{T_{2 n}}=\left\{i_{1}, i_{4}\right\}$ and

$$
\phi_{T_{2 n}}(y) \leqslant f\left(U_{1}\right) \rho_{2 n-1} \int_{\mathbb{R}^{2}} \mathrm{p}_{W_{2 n}^{1}}\left(z_{i_{1}}-y_{i_{2}}\right) \mathrm{p}_{W_{2 n}^{2}}\left(z_{i_{1}}-y_{i_{3}}\right) \mathrm{p}_{U_{2 n}}\left(z_{i_{1}}-y_{i_{3}}\right)
$$




$$
\times \mathrm{p}_{U_{2 n}}\left(z_{i_{1}}-y_{i_{1}}\right) d z_{i_{1}} \delta_{0}\left(y_{i_{4}}-y_{i_{3}}\right) .
$$

Case 3. $i_{3}$ switches via $i_{4}\left(i_{3}\right.$ switches via $i_{2}$ is similar). Then $I_{T_{2 n}}=\left\{i_{1}, i_{3}\right\}$ and

$$
\begin{aligned}
\phi_{T_{2 n}}(y) \leqslant & f\left(U_{1}\right) \rho_{2 n-1} \int_{\mathbb{R}^{2}} \mathrm{p}_{W_{2 n}^{1}}\left(z_{i_{1}}-y_{i_{2}}\right) \mathrm{p}_{W_{2 n}^{2}}\left(z_{i_{1}}-y_{i_{4}}\right) \mathrm{p}_{U_{2 n}}\left(z_{i_{1}}-y_{i_{4}}\right) \\
& \times \mathrm{p}_{U_{2 n}}\left(z_{i_{1}}-y_{i_{1}}\right) d z_{i_{1}} \delta_{0}\left(y_{i_{3}}-y_{i_{4}}\right) .
\end{aligned}
$$

Now we may define $\left\{i_{j}^{2 n}\right\}$ and $\widetilde{V}_{2 n}^{i}\left(=W_{2 n}^{1}, W_{2 n}^{2}\right.$ or $\left.U_{2 n}\right) \geqslant U_{2 n}$, and use $W_{2 n}^{i} \geqslant U_{2 n-1}+$ $U_{2 n}$ to combine these three cases into the simple bound (write $i_{j}$ for $i_{j}^{2 n}$ now),

$$
\begin{aligned}
\phi_{T_{2 n}}(y) \leqslant & f\left(U_{1}\right) \rho_{2 n-1}\left(c_{0} /\left(U_{2 n-1}+U_{2 n}\right)\right) \int_{\mathbb{R}^{2}} \mathrm{p}_{\widetilde{V}_{2 n}^{1}}\left(z_{i_{1}}-y_{i_{2}}\right) \mathrm{p}_{\widetilde{V}_{2 n}^{2}}\left(z_{i_{1}}-y_{i_{4}}\right) \\
& \times \mathrm{p}_{U_{2 n}}\left(z_{i_{1}}-y_{i_{1}}\right) d z_{i_{1}} \delta_{0}\left(y_{i_{3}}-y_{i_{4}}\right), \\
I_{T_{2 n}}= & \left\{i_{1}, i_{3}\right\} .
\end{aligned}
$$

Therefore if $T_{2 n}<t<T_{2 n+1}$, then $I_{t}=\left\{i_{1}, i_{3}\right\}$ and

$$
\begin{aligned}
\phi_{t}(y) \leqslant & f\left(U_{1}\right) \rho_{2 n-1}\left(c_{0} /\left(U_{2 n-1}+U_{2 n}\right)\right) \\
& \times \int_{\mathbb{R}^{2}} \int_{\mathbb{R}^{2}} \mathrm{p}_{\widetilde{V}_{2 n}^{1}+t-T_{2 n}}\left(z_{i_{1}}-y_{i_{2}}\right) \mathrm{p}_{\widetilde{V}_{2 n}^{2}}\left(z_{i_{1}}-z_{i_{4}}\right) \\
& \times \mathrm{p}_{U_{2 n}+t-T_{2 n}}\left(z_{i_{1}}-y_{i_{1}}\right) \mathrm{p}_{t-T_{2 n}}\left(y_{i_{3}}-z_{i_{4}}\right) \mathrm{p}_{t-T_{2 n}}\left(y_{i_{4}}-z_{i_{4}}\right) d z_{i_{1}} d z_{i_{4}} .
\end{aligned}
$$

Use (5.7) and the fact that $\widetilde{V}_{2 n}^{i} \geqslant U_{2 n}$ and argue again as in the derivation of (5.8) to see that

$$
\begin{aligned}
\int_{\mathbb{R}^{2}} \mathrm{p}_{\widetilde{V}_{2 n}^{1}}+t-T_{2 n} & \left(z_{i_{1}}-y_{i_{2}}\right) \mathrm{p}_{\widetilde{V}_{2 n}^{2}}\left(z_{i_{1}}-z_{i_{4}}\right) \mathrm{p}_{U_{2 n}+t-T_{2 n}}\left(z_{i_{1}}-y_{i_{1}}\right) d z_{i_{1}} \\
\leqslant & \int_{\mathbb{R}^{2}}\left[1\left(\left|z_{i_{1}}-y_{i_{2}}\right|<\left|z_{i_{1}}-y_{i_{1}}\right|\right) \mathrm{p}_{\widetilde{V}_{2 n}^{1}+t-T_{2 n}}(0) \mathrm{p}_{U_{2 n}+t-T_{2 n}}\left(\left(y_{i_{1}}-y_{i_{2}}\right) / 2\right)\right. \\
& \left.+1\left(\left|z_{i_{1}}-y_{i_{1}}\right|<\left|z_{i_{1}}-y_{i_{2}}\right|\right) \mathrm{p}_{U_{2 n}+t-T_{2 n}}(0) \mathrm{p}_{\widetilde{V}_{2 n}^{1}+t-T_{2 n}}\left(\left(y_{i_{1}}-y_{i_{2}}\right) / 2\right)\right] \\
& \quad \times \mathrm{p}_{\widetilde{V}_{2 n}^{2}}\left(z_{i_{1}}-z_{i_{4}}\right) d z_{i_{1}} \\
\leqslant & \left(c_{0} /\left(U_{2 n}+t-T_{2 n}\right)\right) \mathrm{p}_{\widetilde{V}_{2 n}^{1}+t-T_{2 n}}\left(\left(y_{i_{1}}-y_{i_{2}}\right) / 2\right) \\
= & 4\left(c_{0} /\left(U_{2 n}+t-T_{2 n}\right)\right) \mathrm{p}_{4 \widetilde{V}_{2 n}^{1}}+4\left(t-T_{2 n}\right)
\end{aligned}
$$

Use this in (5.9) to conclude that for $T_{2 n}<t<T_{2 n+1}, \pi_{t}=\left\{i_{1}, i_{3}\right\}$ and

$$
\begin{aligned}
\phi_{t}(y) \leqslant & f\left(U_{1}\right) \rho_{2 n-1}\left(\frac{2 c_{0}}{U_{2 n-1}+U_{2 n}}\right)\left(\frac{2 c_{0}}{U_{2 n}+t-T_{2 n}}\right) \\
& \times \mathrm{p}_{4 \widetilde{V}_{2 n}^{1}+4\left(t-T_{2 n}\right)}\left(y_{i_{1}}-y_{i_{2}}\right) \mathrm{p}_{2\left(t-T_{2 n}\right)}\left(y_{i_{3}}-y_{i_{4}}\right) \\
= & \rho(t) \mathrm{p}_{4 \widetilde{V}_{2 n}^{1}+4\left(t-T_{2 n}\right)}\left(y_{i_{1}}-y_{i_{2}}\right) \mathrm{p}_{2\left(t-T_{2 n}\right)}\left(y_{i_{3}}-y_{i_{4}}\right) .
\end{aligned}
$$

This gives $(I)_{n}(a)$ and the induction is complete. 
Now we are ready to complete the

Proof of Theorem 5.1. - Fix $0<\delta<T<\infty$. Let $0<p<1 / 2, N>0$ and define

$$
\psi_{N}\left(\mathbf{X}_{\delta}\right)=1\left(\max _{1 \leqslant i, j \leqslant 2} \sup _{0<s \leqslant T} s^{p / 2} \iint \mathrm{p}_{s}\left(x_{1}-x_{2}\right) X_{\delta}^{i}\left(d x_{1}\right) X_{\delta}^{j}\left(d x_{2}\right) \leqslant N\right) \equiv 1_{K_{N}}\left(\mathbf{X}_{\delta}\right) .
$$

We claim first that it suffices to show:

$$
\sup _{\varepsilon>0} P\left[\int_{\delta}^{T} H_{\varepsilon}\left(\mathbf{X}_{s}\right) d s \psi_{N}\left(\mathbf{X}_{\delta}\right)\right]<\infty
$$

where

$$
\begin{aligned}
H_{\varepsilon}\left(\mathbf{X}_{s}\right) \equiv H_{\varepsilon, \mathbb{R}^{2}}\left(\mathbf{X}_{s}\right)= & \int_{\mathbb{R}^{2}} \int_{\mathbb{R}^{2}}\left(1+|x-y|^{-1}\right) S_{\varepsilon} X_{s}^{1}(x) S_{\varepsilon} X_{s}^{2}(x) \\
& \times S_{\varepsilon} X_{s}^{1}(y) S_{\varepsilon} X_{s}^{2}(y) d x d y, \quad \varepsilon>0 .
\end{aligned}
$$

Assume (5.10) and recall that $\mathbf{X}_{\delta} \in \mathcal{M}_{\mathrm{f}, \mathrm{se}}$, a.s., (by Proposition 25(a) of [3]). The latter implies that $P\left(\mathbf{X}_{\delta} \in K_{N}\right) \uparrow 1$ as $N \rightarrow \infty$. Therefore

$$
\begin{aligned}
& P\left(P\left[\int_{\delta}^{T} H_{\varepsilon}\left(\mathbf{X}_{s}\right) d s \mid \mathcal{F}_{\delta}\right]>M\right) \\
& \quad \leqslant P\left(\mathbf{X}_{\delta} \in K_{N}^{c}\right)+P\left(P\left[\int_{\delta}^{T} H_{\varepsilon}\left(\mathbf{X}_{s}\right) d s \psi_{N}\left(\mathbf{X}_{\delta}\right) \mid \mathcal{F}_{\delta}\right]>M\right) \\
& \quad \leqslant P\left(\mathbf{X}_{\delta} \in K_{N}^{c}\right)+M^{-1} P\left[\int_{\delta}^{T} H_{\varepsilon}\left(\mathbf{X}_{s}\right) d s \psi_{N}\left(\mathbf{X}_{\delta}\right)\right] .
\end{aligned}
$$

If $\eta>0$ we may first choose $N>0$ such that the first term is at most $\eta / 2$ and then choose $M$ large enough so that the second term is at most $\eta / 2$ for all $\varepsilon>0$. This proves (IntC).

Turning to (5.10), a change of variables shows that

$$
\int_{0}^{1} u^{-1 / 2}\left(1+\mathrm{p}_{u}(x)\right) d u \geqslant c(\sigma)\left(|x|^{-1}+1\right)
$$

If $\varepsilon>0, u \in(0,1]$, and

$$
\begin{aligned}
\phi_{0}^{u, \varepsilon}(y) & =\phi_{0}\left(y_{1}, y_{2}, y_{3}, y_{4}\right) \\
& =\iint\left(1+\mathrm{p}_{u}(x-y)\right) \prod_{i=1}^{2} \mathrm{p}_{\varepsilon}\left(y_{i}-x\right) \prod_{j=3}^{4} \mathrm{p}_{\varepsilon}\left(y_{j}-y\right) d x d y,
\end{aligned}
$$

then (5.10) is therefore equivalent to 


$$
\begin{aligned}
& \sup _{\varepsilon>0} \int_{0}^{1} \int_{\delta}^{T} P\left[\int \phi_{0}^{u, \varepsilon}\left(y_{1}, y_{2}, y_{3}, y_{4}\right)\right. \\
& \left.\quad \times X_{t}^{1}\left(d y_{1}\right) X_{t}^{1}\left(d y_{3}\right) X_{t}^{2}\left(d y_{2}\right) X_{t}^{2}\left(d y_{4}\right) \psi_{N}\left(\mathbf{X}_{\delta}\right)\right] u^{-1 / 2} d u d t<\infty .
\end{aligned}
$$

If $\left(\phi_{t}, I_{t}\right)$ is the dual process starting at $\left(\phi_{0}^{u, \varepsilon},\{1,3\}\right)$, then for $t<T_{1}$,

$$
\begin{aligned}
\phi_{t}(y)= & \mathrm{p}_{2(\varepsilon+t)}\left(y_{1}-y_{2}\right) \mathrm{p}_{2(\varepsilon+t)}\left(y_{3}-y_{4}\right) \\
& +\iint \mathrm{p}_{u}(x-y) \prod_{i=1}^{2} \mathrm{p}_{\varepsilon+t}\left(y_{i}-x\right) \prod_{j=3}^{4} \mathrm{p}_{\varepsilon+t}\left(y_{j}-y\right) d x d y .
\end{aligned}
$$

Use the fact that $\left|x-y_{1}\right|<\left|x-y_{2}\right|$ implies $\left|y_{1}-y_{2}\right| / 2 \leqslant\left|x-y_{2}\right|$ to bound the second term by

$$
\begin{aligned}
\iint[ & 1\left(\left|x-y_{1}\right|<\left|x-y_{2}\right|\right) \mathrm{p}_{\varepsilon+t}\left(x-y_{1}\right) \mathrm{p}_{\varepsilon+t}\left(\left(y_{1}-y_{2}\right) / 2\right) \\
& \left.+1\left(\left|x-y_{1}\right|>\left|x-y_{2}\right|\right) \mathrm{p}_{\varepsilon+t}\left(x-y_{2}\right) \mathrm{p}_{\varepsilon+t}\left(\left(y_{1}-y_{2}\right) / 2\right)\right] \\
& \times \mathrm{p}_{u}(x-y) \mathrm{p}_{\varepsilon+t}\left(y_{3}-y\right) \mathrm{p}_{\varepsilon+t}\left(y_{4}-y\right) d x d y \\
\leqslant & \mathrm{p}_{\varepsilon+t}\left(\left(y_{1}-y_{2}\right) / 2\right) \int\left(\mathrm{p}_{\varepsilon+t+u}\left(y-y_{1}\right)+\mathrm{p}_{\varepsilon+t+u}\left(y-y_{2}\right)\right) \\
& \times \mathrm{p}_{\varepsilon+t}\left(y_{3}-y\right) \mathrm{p}_{\varepsilon+t}\left(y_{4}-y\right) d y \\
\leqslant & \frac{2 c_{0}}{\varepsilon+t+u} 4 \mathrm{p}_{4(\varepsilon+t)}\left(y_{1}-y_{2}\right) \mathrm{p}_{2(\varepsilon+t)}\left(y_{3}-y_{4}\right) .
\end{aligned}
$$

Put this into (5.13) to see that for $0 \leqslant t<T_{1}$,

$$
\phi_{t}(y) \leqslant\left(2+\frac{8 c_{0}}{\varepsilon+t+u}\right) \mathrm{p}_{4(\varepsilon+t)}\left(y_{1}-y_{2}\right) \mathrm{p}_{2(\varepsilon+t)}\left(y_{3}-y_{4}\right)
$$

This shows the hypothesis (5.4) of Lemma 5.2 holds with $f(t)=2+\frac{8 c_{0}}{t+u}$.

If $\rho(t)$ is defined as in Lemma 5.2, then that result and $(\mathrm{MB})_{\delta}$ imply

$$
\begin{aligned}
& \int_{\delta}^{T} P\left[\int \phi_{0}^{u, \varepsilon}(y) X_{t}^{1}\left(d y_{1}\right) X_{t}^{1}\left(d y_{3}\right) X_{t}^{2}\left(d y_{2}\right) X_{t}^{2}\left(d y_{4}\right) \psi_{N}\left(\mathbf{X}_{\delta}\right)\right] d t \\
& \quad \leqslant \int_{\delta}^{T} \widehat{P}_{\phi_{0}^{u, \varepsilon}, I_{0}} \times P\left[\int \phi_{t-\delta}(y) \prod_{i \in I_{t-\delta}} X_{\delta}^{1}\left(d y_{i}\right) \prod_{j \notin I_{t-\delta}} X_{\delta}^{2}\left(d y_{j}\right) \psi_{N}\left(\mathbf{X}_{\delta}\right)\right] \mathrm{e}^{3 \gamma T} d t \\
& \quad \leqslant \int_{\delta}^{T} \mathrm{e}^{3 \gamma T} \sum_{n=0}^{\infty} \widehat{P}_{\phi_{0}^{u, \varepsilon}, I_{0}} \times P\left[1\left(T_{n}<t<T_{n+1}\right) 2 \rho(t)\left(t-T_{n}\right)^{-p}\right] N^{2} d t \\
& \quad \leqslant c(\gamma, T, N) \int_{\delta}^{T}\left\{\widehat{P}_{\phi_{0}^{u, \varepsilon}, I_{0}}\left[1\left(t<T_{1}\right)\left(1+(t+u)^{-1}\right) t^{-p}\right]\right.
\end{aligned}
$$




$$
\begin{aligned}
& +\sum_{n=1}^{\infty} \widehat{P}_{\phi_{0}^{u, \varepsilon}, I_{0}}\left[1\left(T_{n}<t<T_{n}+U_{n+1}\right)\left(1+\frac{1}{u+U_{1}}\right)\right. \\
& \left.\left.\times \prod_{k=1}^{n-1}\left(U_{k}+U_{k+1}\right)^{-1}\left(U_{n}+t-T_{n}\right)^{-1}\left(t-T_{n}\right)^{-p}\right] c_{1}^{n}\right\} d t .
\end{aligned}
$$

Recall (5.1), write out the appropriate exponential densities, and set $u_{0}=u$ to bound (5.15) by $(c(\gamma, T, n)$ may change from line to line)

$$
\begin{aligned}
& c(\gamma, T, N)\left\{\int_{\delta}^{T}\left(1+(t+u)^{-1}\right) t^{-p} d t\right. \\
& \quad+\sum_{n=1}^{\infty} 6^{n / 2} \gamma^{n} c_{1}^{n} \int_{\delta} \int_{\mathbb{R}_{+}^{n}} 1\left(\sum_{i=1}^{n} u_{i}<t\right)\left(1+\frac{1}{u_{0}+u_{1}}\right) \prod_{k=1}^{n-1}\left(u_{k}+u_{k+1}\right)^{-1} \\
&\left.\quad \times\left(u_{n}+t-\sum_{i=1}^{n} u_{i}\right)^{-1}\left(t-\sum_{i=1}^{n} u_{i}\right)^{-p} d u_{1} \ldots d u_{n} d t\right\} \\
& \leqslant c(\gamma, T, N)\left\{1+\int_{0}^{T}(t+u)^{-1} t^{-p} d t+\sum_{n=1}^{\infty}\left(\sqrt{6} \gamma c_{1}\right)^{n} \int_{\mathbb{R}_{+}^{n+1}} 1\left(\sum_{i=1}^{n+1} u_{i}<T\right)\right. \\
&\left.\times\left(\prod_{k=1}^{n}\left(u_{k}+u_{k+1}\right)^{-1}+\prod_{k=0}^{n}\left(u_{k}+u_{k+1}\right)^{-1}\right) u_{n+1}^{-p} d u_{1} \ldots d u_{n+1}\right\} .
\end{aligned}
$$

If $c(p)=\pi(\sin ((1-p) \pi))^{-1}$ and

$$
I_{n, p}\left(u_{0}\right)=\int_{\mathbb{R}_{+}^{n}}\left(u_{0}+u_{1}\right)^{-1} \prod_{j=1}^{n-1}\left(u_{j}+u_{j+1}\right)^{-1} u_{n}^{-p} d u_{1} \ldots d u_{n}
$$

then (5.16) is at most

$$
\begin{aligned}
& c(\gamma, T, N)\left\{1+I_{1, p}(u)+\sum_{n=1}^{\infty}\left(\sqrt{6} \gamma c_{1}\right)^{n}\left(\int_{0}^{T} I_{n, p}\left(u_{1}\right) d u_{1}+I_{n+1, p}(u)\right)\right\} \\
& =c(\gamma, T, N)\left\{1+c(p) u^{-p}\right. \\
& \left.\quad+\sum_{n=1}^{\infty}\left(\sqrt{6} \gamma c_{1} c(p)\right)^{n}\left(\int_{0}^{T} u_{1}^{-p} d u_{1}+c(p) u^{-p}\right)\right\}, \quad \text { (by Lemma } 60 \text { of [3]) } \\
& \leqslant c(\gamma, T, N, p, \sigma)\left(1+u^{-p}\right),
\end{aligned}
$$

where we used $(a)$ in the last line and have chosen $p<1 / 2$, sufficiently close to $1 / 2$ so that $\sqrt{6} c_{1} \gamma c(p)<1$. Put the above upper bound for (5.14) into (5.12) (and recall (5.11) to conclude that 


$$
\begin{aligned}
\sup _{\varepsilon>0} P\left[\int_{\delta}^{T} H_{\varepsilon}\left(\mathbf{X}_{s}\right) d s \psi_{N}\left(\mathbf{X}_{\delta}\right)\right] & \leqslant c(\sigma)^{-1} c(\gamma, T, N, p, \sigma) \int_{0}^{1} u^{-1 / 2}\left(1+u^{-p}\right) d u \\
& \equiv c(\gamma, T, N, p, \sigma)<\infty
\end{aligned}
$$

since $p<1 / 2$. This concludes the proof of (a).

(b) This is the minor modification of the above. We need the estimate (5.12) with $\delta=0$ and $\psi_{N}=1$. The only change is in the derivation of (5.15) where instead of using $\psi_{N}$ to get a factor of $N^{2}$ we simply use $\mathbf{X}_{0} \in \mathcal{M}_{\mathrm{f} \text {,se }}$ to get a constant depending on $\mathbf{X}_{0}$. The rest of the proof is the same.

\subsection{Existence of collision measure}

TheOrem 5.3. - Let $\mathbf{X}_{0} \in \mathcal{M}_{\mathrm{f}, \mathrm{e}}$ and $\mathbf{X}$ be the unique in law solution of $(\mathbf{M P})_{\mathbf{X}_{0}}^{\sigma, \gamma}$ satisfying (IntC) on $\left(\Omega, \mathcal{F}, \mathcal{F}_{t}, P\right)$. There is a jointly measurable map

$$
K_{\mathbf{X}}: \mathbb{R}_{+} \times \Omega \mapsto \mathcal{M}_{\mathrm{f}}\left(\mathbb{R}^{2}\right)
$$

such that

$$
L_{\mathbf{X}}(t, \cdot)=\int_{0}^{t} K_{\mathbf{X}}(s, \cdot) d s, \quad \forall t \geqslant 0, P \text {-a.s. }
$$

Proof. - We work with $(\widehat{\Omega}, \widehat{\mathcal{F}}, \widehat{P})=(\Omega \times[0,1], \mathcal{F} \times \mathcal{B}, P \times d x)$ on which we define $\mathcal{M}_{\mathrm{f}}\left(\mathbb{R}^{2}\right)$-valued random vectors $Z^{\delta}$ (for $\delta>0$ ) by

$$
Z^{\delta}(w, s)=\frac{L_{\mathbf{X}}(s+\delta)-L_{\mathbf{X}}(s)}{\delta} .
$$

Let $\varphi \in \mathcal{C}_{\mathrm{b}}^{+}$. Then $s \mapsto L_{\mathbf{X}}(s)(\varphi)$ is a continuous non-decreasing process and so by standard differentiation theory

$$
\left\langle Z^{\delta}, \varphi\right\rangle \rightarrow\left\langle L_{\mathbf{X}}^{\prime}(s), \varphi\right\rangle \equiv\left\langle K_{\mathbf{X}}(s), \varphi\right\rangle, \quad \widehat{P} \text {-a.s., as } \delta \downarrow 0 .
$$

By Proposition 52 of [3] $\left\langle L_{\mathbf{X}}^{*, \eta}(s), 1\right\rangle \rightarrow\left\langle L_{\mathbf{X}}(s), 1\right\rangle$ in $P$-probability as $\eta \downarrow 0$ for each $s \geqslant 0$.

Now let us fix arbitrary $u \in(0,1)$. (IntC) implies that there exists a sequence $F_{1} \subset F_{2} \subset \cdots$ of events in $\mathcal{F}_{u}$ such that $F_{l} \uparrow \Omega$, as $l \rightarrow \infty$ and for each $n \geqslant 1$ :

$$
\limsup _{\varepsilon \downarrow 0} P\left[\int_{u}^{t} H_{\varepsilon}\left(\mathbf{X}_{s}\right) d s 1_{F_{n}}\right]<\infty, \quad \forall t \geqslant u .
$$

For each $l \geqslant 1$ define the measure $P_{l}$ on $\Omega$ by

$$
P_{l}(B)=P\left(1_{F_{l}} 1_{B}\right), \quad B \in \mathcal{F},
$$


and for each $u \in(0,1)$ define $\widehat{P}_{l, u}$ on $\Omega \times[0,1]$ by

$$
\widehat{P}_{l, u}(B)=\widehat{P}\left(1_{F_{l} \times[u, 1]} 1_{B}\right), \quad B \in \mathcal{F} \times \mathcal{B} .
$$

In the following denote $L_{t} \equiv L_{\mathbf{X}}(t), K_{t} \equiv K_{\mathbf{X}}(t)$.

Fix $1>\delta>0$. Then we may choose $\eta_{n} \downarrow 0$ so that

$$
\delta^{-1}\left\langle L_{s+\delta}^{*, \eta_{n}}(\omega)-L_{s}^{*, \eta_{n}}(\omega), 1\right\rangle \rightarrow\left\langle Z^{\delta}(\omega, s), 1\right\rangle, \quad \widehat{P} \text {-a.s. as } n \rightarrow \infty .
$$

Fix some $l \geqslant 1$ and $u \in(0,1) . \widehat{P}_{l, u}$ is absolutely continuous with respect to $\widehat{P}$, therefore

$$
\delta^{-1}\left\langle L_{s+\delta}^{*, \eta_{n}}(\omega)-L_{s}^{*, \eta_{n}}(\omega), 1\right\rangle \rightarrow\left\langle Z^{\delta}(\omega, s), 1\right\rangle, \quad \widehat{P}_{l, u} \text {-a.s. as } n \rightarrow \infty .
$$

Therefore Fatou's lemma and Jensen's inequality imply

$$
\begin{aligned}
\widehat{P}_{l, u}\left[\left\langle Z^{\delta}, \varphi\right\rangle^{2}\right] & \leqslant\|\varphi\|_{\infty}^{2} \liminf _{n \rightarrow \infty} \widehat{P}_{l, u}\left[\left\langle L_{\cdot+\delta}^{*, \eta_{n}}(\cdot)-L^{*, \eta_{n}}(\cdot), 1\right\rangle^{2}\right] \delta^{-2} \\
& =\|\varphi\|_{\infty}^{2} \liminf _{n \rightarrow \infty} P_{l}\left[\int_{u}^{1}\left(\int_{s}^{s+\delta} \int_{0}^{\eta_{n}} \int_{\mathbb{R}^{2}} S_{r} X_{v}^{1}(x) S_{r} X_{v}^{2}(x) d x \frac{d r}{\eta_{n}} \frac{d v}{\delta}\right)^{2} d s\right] \\
& \leqslant\|\varphi\|_{\infty}^{2} \liminf _{n \rightarrow \infty} P_{l}\left[\int_{u}^{1} \int_{s}^{s+\delta} \int_{0}^{2}\left(\int_{\mathbb{R}^{2}} S_{r} X_{v}^{1}(x) S_{r} X_{v}^{2}(x) d x\right)^{2} \frac{d v}{\eta_{n}} d s\right] \\
& \leqslant\|\varphi\|_{\infty}^{2} \liminf _{n \rightarrow \infty} \int_{0}^{\eta_{n}} P_{l}\left[\int_{u}^{1+\delta}\left(\int_{\mathbb{R}^{2}} S_{r} X_{v}^{1}(x) S_{r} X_{v}^{2}(x) d x\right)^{2} d v\right] \frac{d r}{\eta_{n}} \\
& \leqslant\|\varphi\|_{\infty}^{2} \liminf _{n \rightarrow \infty} \int_{0}^{\eta_{n}} P\left[\int_{u}^{2} H_{r}\left(\mathbf{X}_{v}\right) d v 1_{F_{l}}\right] \frac{d r}{\eta_{n}} \\
& \leqslant\|\varphi\|_{\infty}^{2} C,
\end{aligned}
$$

by (5.19) independent of $\delta$. This together with (5.18) shows that

$$
\left\langle Z^{\delta}, \varphi\right\rangle \rightarrow K_{s}(\varphi), \quad \text { in } L^{1}\left(\widehat{P}_{l, u}\right) \text { as } \delta \downarrow 0 .
$$

Therefore

$$
\begin{aligned}
P\left[\int_{u}^{1} K_{s}(\varphi) d s 1_{F_{l}}\right] & =\lim _{\delta \downarrow 0} \widehat{P}_{l, u}\left[\left\langle Z^{\delta}, \varphi\right\rangle\right] \\
& =\lim _{\delta \downarrow 0} P_{l}\left[\int_{u}^{1}\left\langle L_{s+\delta}, \varphi\right\rangle-\left\langle L_{s}, \varphi\right\rangle d s\right] \delta^{-1} \\
& =\lim _{\delta \downarrow 0} P_{l}\left[\int_{1}^{1+\delta}\left\langle L_{s+\delta}, \varphi\right\rangle d s \delta^{-1}-\int_{u}^{u+\delta}\left\langle L_{s}, \varphi\right\rangle d s \delta^{-1}\right]
\end{aligned}
$$




$$
=P\left[\left(\left\langle L_{1}, \varphi\right\rangle-\left\langle L_{u}, \varphi\right\rangle\right) 1_{F_{l}}\right]
$$

where the last equality follows by continuity of $\left\langle L_{1}, \varphi\right\rangle$ and fact that $\left\langle L_{t}, \varphi\right\rangle$ is $P$ integrable. Now take $l \rightarrow \infty$. Then by Monotone Convergence we get from (5.21)

$$
P\left[\int_{u}^{1} K_{s}(\varphi) d s\right]=P\left[\left\langle L_{1}, \varphi\right\rangle-\left\langle L_{u}, \varphi\right\rangle\right] .
$$

Now let $u \rightarrow 0$, use Monotone Convergence on the left side again, and continuity of $L_{t}$ and Dominated Convergence on the right side to get

$$
P\left[\int_{0}^{1} K_{s}(\varphi) d s\right]=P\left[\left\langle L_{1}, \varphi\right\rangle\right] .
$$

This equality implies that the singular part of $s \mapsto L_{s}(\varphi)$ is $P$-a.s. 0 and so,

$$
\left\langle L_{t}, \varphi\right\rangle=\int_{0}^{t} K_{s}(\varphi) d s, \quad \forall t \in[0,1] P \text {-a.s. } \forall \varphi \in \mathcal{C}_{\mathrm{b}}^{+} .
$$

It remains to choose a version of $K(\omega, s)$ which is a finite measure on $\mathbb{R}^{2}$. First note that by the same arguments as in (5.21) we can see that

$$
\lim _{\delta_{n} \downarrow 0} \widehat{P}\left[\left\langle Z^{\delta_{n}}, \varphi\right\rangle\right]=P\left[\left\langle L_{1}, \varphi\right\rangle\right] .
$$

Since $P\left[L_{1}(d x)\right]$ is a finite measure on $\mathbb{R}^{2}$ we can easily get that the sequence of measures $\left\{Z^{\delta_{n}}\right\}$ is tight in $\mathcal{M}_{\mathrm{f}}, \widehat{P}$-a.s. Let $\left\{\varphi_{k}\right\}$ be a countable determining class in $\mathcal{C}_{\mathrm{b}}^{+}$. By considering an appropriate subsequence $\delta_{n}$ in (5.18) we may assume $\lim _{\delta_{n} \downarrow 0}\left\langle Z^{\delta_{n}}, \varphi_{k}\right\rangle=$ $K\left(\varphi_{k}\right), \forall k \widehat{P}$-a.s. It follows that there is a random measure $\widetilde{K} \in \mathcal{M}_{\mathrm{f}}$ on $(\widehat{\Omega}, \widehat{\mathcal{F}})$ such that $Z^{\delta_{n}} \rightarrow \widetilde{K} \widehat{P}$-a.s. and $K(\varphi)=\langle\widetilde{K}, \varphi\rangle \widehat{P}$-a.s. $\forall \varphi \in \mathcal{C}_{\mathrm{b}}$. The required result is now clear from (5.23), at least for $t \in[0,1]$. Simply repeat the above construction on $[i, i+1] \forall i \in \mathbb{N}$ to complete the proof.

\subsection{Proof of Proposition 1.14}

Let $\mathbf{X}$ be any solution to $(\mathbf{M P})_{\mathbf{X}_{0}}^{\sigma, \gamma}$ satisfying $(\mathbf{I n t C})$ on $\left(\Omega, \mathcal{F}, \mathcal{F}_{t}, P\right)$ for some $\mathcal{F}_{0}$ measurable initial condition $X_{0} \in \mathcal{M}_{\mathrm{f}, \mathrm{e}}$ satisfying $(\mathbf{E n C})$. Let $\widetilde{\mathbf{X}}_{0}=\left(\tilde{x}_{0}^{1} d x, \tilde{x}_{0}^{2} d x\right)$ where $\left(\tilde{x}_{0}^{1}, \tilde{x}_{0}^{2}\right) \in\left(\mathcal{C}_{\mathrm{b}}^{+}\right)^{2}$. Take $\varphi^{n}=\left(\varphi^{1, n}, \varphi^{2, n}\right) \in\left(\mathcal{C}_{\mathrm{com}}^{+}\right)^{2}, n \geqslant 1$ such that $\varphi^{i, n} \uparrow \tilde{x}_{0}^{i}, i=1,2$, pointwise. Let $\widetilde{\mathbf{X}}$ and $\widetilde{\mathbf{X}}^{n}$ be particular solutions of $(\mathbf{M P})_{\widetilde{\mathbf{X}}_{0}}^{\sigma, \gamma}$ and $(\mathbf{M P})_{\varphi^{n}}^{\sigma, \gamma}$ respectively, constructed in Section 1.4 on some probability space $\left(\widetilde{\Omega}, \widetilde{\mathcal{F}}, \widetilde{\mathcal{F}}_{t}, \widetilde{P}\right)$.

Then

$$
\begin{aligned}
& \left|P\left[\mathfrak{E}\left(\mathbf{X}_{t}, \widetilde{\mathbf{X}}_{0}\right)\right]-\lim _{\varepsilon \downarrow 0} P \times \widetilde{P}\left[\mathfrak{E}\left(\mathbf{X}_{0}, S_{\varepsilon} \widetilde{\mathbf{X}}_{t}\right)\right]\right| \\
& \quad \leqslant\left|P\left[\mathfrak{E}\left(\mathbf{X}_{t}, \widetilde{\mathbf{X}}_{0}\right)\right]-P\left[\mathfrak{E}\left(\mathbf{X}_{t}, \varphi^{n}\right)\right]\right|
\end{aligned}
$$




$$
\begin{aligned}
& +\left|\lim _{\varepsilon \downarrow 0} P \times \widetilde{P}\left[\mathfrak{E}\left(\mathbf{X}_{0}, S_{\varepsilon} \widetilde{\mathbf{X}}_{t}^{n}\right)\right]-P \times \widetilde{P}\left[\mathfrak{E}\left(\mathbf{X}_{0}, S_{\delta} \widetilde{\mathbf{X}}_{t-\delta}^{n}\right)\right]\right| \\
& +\left|P \times \widetilde{P}\left[\mathfrak{E}\left(\mathbf{X}_{0}, S_{\delta} \widetilde{\mathbf{X}}_{t-\delta}^{n}\right)\right]-P \times \widetilde{P}\left[\mathfrak{E}\left(\mathbf{X}_{0}, S_{\delta} \widetilde{\mathbf{X}}_{t-\delta}\right)\right]\right| \\
& +\left|P \times \widetilde{P}\left[\mathfrak{E}\left(\mathbf{X}_{0}, S_{\delta} \widetilde{\mathbf{X}}_{t-\delta}\right)\right]-\lim _{\varepsilon \downarrow 0} P \times \widetilde{P}\left[\mathfrak{E}\left(\mathbf{X}_{0}, S_{\varepsilon} \widetilde{\mathbf{X}}_{t}\right)\right]\right| \quad \text { (by Proposition 1.12) } \\
\leqslant & 4 \gamma\left(\left|\varphi^{1, n}\right|_{\infty}\left|\varphi^{2, n}\right|_{\infty}+\left|\tilde{x}_{0}^{1}\right|_{\infty}\left|\tilde{x}_{0}^{2}\right|_{\infty}\right) P\left[\int_{\mathbb{R}^{2}} \bar{g}_{\delta}\left(X_{0}^{1}, X_{0}^{2}\right)(x) d x\right] \quad \text { (by Lemma 4.1(b)) } \\
& +\left|P\left[\mathfrak{E}\left(\mathbf{X}_{t}, \widetilde{\mathbf{X}}_{0}\right)\right]-P\left[\mathfrak{E}\left(\mathbf{X}_{t}, \varphi^{n}\right)\right]\right|+\mid P \times \widetilde{P}\left[\mathfrak{E}\left(\mathbf{X}_{0}, S_{\delta} \widetilde{\mathbf{X}}_{t-\delta}^{n}\right)\right] \\
& -P \times \widetilde{P}\left[\mathfrak{E}\left(\mathbf{X}_{0}, S_{\delta} \widetilde{\mathbf{X}}_{t-\delta}\right)\right] \mid \\
= & I^{1, \delta, n}+I^{2, \delta, n}+I^{3, \delta, n}
\end{aligned}
$$

for any $0<\delta<t$ and $n \geqslant 1$. Now take $n \rightarrow \infty$. Then by Proposition 4.2 we get that $\widetilde{\mathbf{X}}_{t-\delta}^{n} \Rightarrow \widetilde{\mathbf{X}}_{t-\delta}$ in $\mathcal{M}_{\text {tem }}^{2}$. Since $S_{\delta} X_{0}^{j}, j=1,2$, is not necessarily in $\mathcal{C}_{\text {exp }}$, we need to check that $\widetilde{X}_{t-\delta}^{j, n}\left(S_{\delta} X_{0}^{j}\right) \Rightarrow \widetilde{X}_{t-\delta}^{j}\left(S_{\delta} X_{0}^{j}\right)$. But this follows easily by $L^{2}(P \times \widetilde{P})$ boundedness of $\tilde{X}_{t-\delta}^{j, n}\left(S_{\delta} X_{0}^{j}\right)$ uniformly in $n$ and the fact that

$$
\begin{aligned}
P \times \widetilde{P}\left[\widetilde{X}_{t-\delta}^{j, n}\left(S_{\delta} X_{0}^{j}\right)\right] & =P\left[\widetilde{X}_{0}^{j, n}\left(S_{t} X_{0}^{j}\right)\right] \\
& \rightarrow P\left[\widetilde{X}_{0}^{j}\left(S_{t} X_{0}^{j}\right)\right]=P \times \widetilde{P}\left[\widetilde{X}_{t-\delta}^{j}\left(S_{\delta} X_{0}^{j}\right)\right]<\infty .
\end{aligned}
$$

These two facts together easily give that $\widetilde{X}_{t-\delta}^{j, n}\left(S_{\delta} X_{0}^{j}\right) \Rightarrow \widetilde{X}_{t-\delta}^{j}\left(S_{\delta} X_{0}^{j}\right)$ (details are left for the reader). Therefore $I^{3, n, \delta} \rightarrow 0$ as $n \rightarrow \infty$. Since $\varphi^{n, j} \rightarrow \tilde{x}_{0}^{j}$ boundedly pointwise we get that $I^{2, n, \delta} \rightarrow 0$ as $n \rightarrow \infty$. The first term $I^{1, n, \delta}$ is bounded by

$$
\begin{aligned}
& \underset{n}{4}\left(\sup _{n}\left|\varphi^{1, n}\right|_{\infty}\left|\varphi^{2, n}\right|_{\infty}+\left|\tilde{x}_{0}^{1}\right|_{\infty}\left|\tilde{x}_{0}^{2}\right|_{\infty}\right) P\left[\int_{\mathbb{R}^{2}} \bar{g}_{\delta}\left(X_{0}^{1}, X_{0}^{2}\right)(x) d x\right] \\
& \quad \leqslant 8 \gamma\left|\tilde{x}_{0}^{1}\right|_{\infty}\left|\tilde{x}_{0}^{2}\right|_{\infty} P\left[\int_{\mathbb{R}^{2}} \bar{g}_{\delta}\left(X_{0}^{1}, X_{0}^{2}\right)(x) d x\right]
\end{aligned}
$$

uniformly in $n$. Now by (EnC) and Dominated Convergence we get $\lim _{\delta \downarrow 0} \sup _{n} I^{1, n, \delta}=0$ and so we are done.

\subsection{Markov and strong Markov properties}

Lemma 5.4. - Let $\widetilde{\mathbf{X}}_{0} \in\left(\mathcal{C}_{\text {rap }}^{+}\right)^{2}$ and $\widetilde{\mathbf{X}}$ be the particular solution of $(\mathbf{M P})_{\widetilde{\mathbf{x}}_{0}}^{\sigma, \gamma}$ constructed in Section 1.4 on some $\left(\widetilde{\Omega}, \widetilde{\mathcal{F}}_{,}, \widetilde{\mathcal{F}}_{t}, \widetilde{P}\right)$.

(a) There is a time-homogeneous Borel Markov transition kernel $\mathrm{P}=\left\{\mathrm{P}_{t}(\mu, d v)\right.$ : $\left.t>0, \mu \in \mathcal{M}_{\mathrm{f}, \mathrm{e}}\right\}$ on $\mathcal{M}_{\mathrm{f}, \mathrm{e}}$ such that

$$
\int_{\mathcal{M}_{\mathrm{f}, \mathrm{e}}} \mathfrak{E}\left(\nu, \widetilde{\mathbf{X}}_{0}\right) \mathrm{P}_{t}(\mu, d \nu)=\lim _{\varepsilon \downarrow 0} \widetilde{P}\left[\mathfrak{E}\left(\mu, S_{\varepsilon}\left(\widetilde{\mathbf{X}}_{t}\right)\right)\right] .
$$


(b) Let $\mathbf{X}$ satisfy (IntC) and (EnC) and solve (MP) $\mathbf{\mathbf { X }}_{0}^{\sigma, \gamma}$. Then for any bounded Borel measurable function $h$ on $\mathcal{M}_{\mathrm{f}, \mathrm{e}}$ and any a.s. finite $\mathcal{F}_{t}$-stopping time $\tau$,

$$
P\left[h\left(\mathbf{X}_{\tau+t}\right) \mid \mathcal{F}_{\tau}\right]=\int_{\mathcal{M}_{\mathrm{f}, \mathrm{e}}} h(v) \mathrm{P}_{t}\left(\mathbf{X}_{\tau}, d v\right) \quad \text { a.s. }
$$

i.e. $\mathbf{X}$ is a strong Markov process.

Proof. -

(a) Fix a (deterministic) $\mathbf{X}_{0} \in \mathcal{M}_{\mathrm{f}, \mathrm{e}}$. By Theorem 1.9(a) for any $\mathbf{X}_{0} \in \mathcal{M}_{\mathrm{f}, \mathrm{e}}$ there exists a solution $\mathbf{X}$ to the martingale problem (MP) $\mathbf{\mathbf { X }}_{0}^{\sigma, \gamma}$ satisfying (IntC) and by Proposition 1.12

$$
P\left[\mathfrak{E}\left(\mathbf{X}_{t}, \widetilde{\mathbf{X}}_{0}\right)\right]=\lim _{\varepsilon \downarrow 0} \widetilde{P}\left[\mathfrak{E}\left(\mathbf{X}_{0}, S_{\varepsilon}\left(\widetilde{\mathbf{X}}_{t}\right)\right)\right] .
$$

By Theorem 1.9(b) the solution $\mathbf{X}$ is unique. Let $\mathrm{P}_{t}\left(\mathbf{X}_{0}, \cdot\right)$ be the unique law of $\mathbf{X}_{t}$. Then (5.26) shows

$$
\int_{\mathcal{M}_{\mathrm{f}, \mathrm{e}}} \mathfrak{E}\left(\nu, \widetilde{\mathbf{X}}_{0}\right) \mathrm{P}_{t}\left(\mathbf{X}_{0}, d v\right)=\lim _{\varepsilon \downarrow 0} \widetilde{P}\left[\mathfrak{E}\left(\mathbf{X}_{0}, S_{\varepsilon}\left(\widetilde{\mathbf{X}}_{t}\right)\right)\right] .
$$

The right side is Borel measurable in $\mathbf{X}_{0} \in \mathcal{M}_{\mathrm{f}, \mathrm{e}}$. A monotone class argument shows the bounded pointwise closure of the complex linear span of $\left\{\mathfrak{E}\left(\cdot, \widetilde{\mathbf{X}}_{0}\right): \widetilde{\mathbf{X}}_{0} \in\left(\mathcal{C}_{\text {rap }}^{+}\right)^{2}\right\}$ is the set of all bounded complex-valued measurable maps in $\mathcal{M}_{\mathrm{f}, \mathrm{e}}$ (e.g. see Lemma 6.2 of [5]). The Borel measurability of $\mathbf{X}_{0} \mapsto \mathrm{P}_{t}\left(\mathbf{X}_{0}, \cdot\right)$ from $\mathcal{M}_{\mathrm{f}, \mathrm{e}}$ to $\mathcal{M}_{1}\left(\mathcal{M}_{\mathrm{f}, \mathrm{e}}\right)$ follows.

(b) We now proceed by modifying the proof of Theorem 4.4.2 of [7] to accommodate the side conditions (IntC) and $(\mathbf{E n C})$. Let $\mathbf{X}$ be the unique solution to $(\mathbf{M P})_{\mathbf{X}_{0}}^{\sigma, \gamma}$ on $\left(\Omega, \mathcal{F}, \mathcal{F}_{t}, P\right)$ satisfying (IntC) with $\mathbf{X}_{0}$ (possibly random) satisfying (EnC). Let $\tau$ be any bounded stopping time and $F \in \mathcal{F}_{\tau}$ with $P(F)>0$. Define

$$
P_{1}(B)=P\left(1_{F} P\left(1_{B} \mid \mathcal{F}_{\tau}\right)\right) / P(F), \quad B \in \mathcal{F},
$$

and $\mathbf{Y}_{s}=\mathbf{X}_{\tau+s}$. Then by Lemma 3.3, $\mathbf{Y}$ solves $(\mathbf{M P})_{Y_{0}}^{\sigma, \gamma}$ on $\left(\Omega, \mathcal{F}, \mathcal{F}_{t}^{\tau}, P_{1}\right)$ with $\mathcal{L}\left(\mathbf{Y}_{0}\right)=$ $P\left(\mathbf{X}_{\tau} \in \cdot \mid F\right)$ and satisfies (IntC) and (EnC). So by Proposition 1.12 if $\widetilde{\mathbf{X}}_{0} \in\left(\mathcal{C}_{\text {rap }}^{+}\right)^{2}$ and $\varrho=\mathcal{L}\left(\mathbf{Y}_{0}\right)$ then

$$
\begin{aligned}
P\left[\mathfrak{E}\left(\mathbf{Y}_{t}, \widetilde{\mathbf{X}}_{0}\right)\right] & =\lim _{\varepsilon \downarrow 0} \int_{\mathcal{M}_{\mathrm{f}, \mathrm{e}}} \widetilde{P}\left[\mathfrak{E}\left(\mu, S_{\varepsilon}\left(\widetilde{\mathbf{X}}_{t}\right)\right)\right] \varrho(d \mu) \\
& =\int_{\mathcal{M}_{\mathrm{f}, \mathrm{e}}} \int_{\mathcal{M}_{\mathrm{f}, \mathrm{e}}} \mathfrak{E}\left(\nu, \widetilde{\mathbf{X}}_{0}\right) \mathrm{P}_{t}(\mu, d \nu) \varrho(d \mu)
\end{aligned}
$$

by (5.27) and Dominated Convergence. A monotone class argument again as in Lemma 6.2 of [5] shows that for any bounded Borel $h: \mathcal{M}_{\mathrm{f}, \mathrm{e}} \mapsto \mathbb{R}$

$$
P_{1}\left[h\left(\mathbf{Y}_{t}\right)\right]=\int_{\mathcal{M}_{\mathrm{f}, \mathrm{e}}} \int_{\mathcal{M}_{\mathrm{f}, \mathrm{e}}} h(v) \mathrm{P}_{t}(\mu, d \nu) \varrho(d \mu)
$$


and so

$$
P\left(1_{F} P\left(h\left(\mathbf{X}_{\tau+t}\right) \mid \mathcal{F}_{\tau}\right)\right)=P\left(1_{F} \int_{\mathcal{M}_{\mathrm{f}, \mathrm{e}}} h(v) \mathrm{P}_{t}\left(\mathbf{X}_{\tau}, d v\right)\right) .
$$

The required result follows for bounded stopping times, and the obvious truncation argument then gives the result for a.s. finite stopping times.

Remark 5.5. - Lemma 5.4 completes the proof of Theorem 1.9(c).

\section{Acknowledgement}

We thank a referee for some useful comments and suggestions which improved the exposition.

\section{Appendix A}

LEMma A.1. - For any $\lambda \in \mathbb{R}$ and $t>0$, set

$$
c_{A 1}(t, \lambda) \equiv \int_{\mathbb{R}^{2}} \frac{1}{2 \pi t \sigma^{2}} \mathrm{e}^{-|y|^{2} / 2 t \sigma^{2}} \mathrm{e}^{|\lambda y|} d y .
$$

Then, for any $\lambda \in \mathbb{R}$ and $\psi \in \mathcal{C}_{\lambda}$, we have

$$
S_{t} \psi(x) \leqslant c_{A 1}(t, \lambda)|\psi|_{\lambda} \phi_{\lambda}(x), \quad \forall x \in \mathbb{R}^{2},
$$

and

$$
\sup _{0 \leqslant t \leqslant T} c_{A 1}(t, \lambda)=c_{A 1}(T, \lambda)<\infty, \quad \forall T>0 .
$$

Proof. - (A.2) is obvious because $\mathrm{e}^{\left|\lambda B_{t}\right|}$ is a submartingale. We will prove (A.1) for $\lambda \geqslant 0$. For a negative $\lambda$ the proof is analogous. For $\lambda \geqslant 0$, we have

$$
\begin{aligned}
\int_{\mathbb{R}^{2}} \mathrm{p}_{t}(x-y) \psi(y) d y & =\int_{\mathbb{R}^{2}} \frac{1}{2 \pi t \sigma^{2}} \mathrm{e}^{-|x-y|^{2} / 2 t \sigma^{2}} \psi(y) d y \\
& \leqslant|\psi|_{\lambda} \int_{\mathbb{R}^{2}} \frac{1}{2 \pi t \sigma^{2}} \mathrm{e}^{-|x-y|^{2} / 2 t \sigma^{2}} \mathrm{e}^{-\lambda|y|} d y \\
& =|\psi|_{\lambda} \mathrm{e}^{-\lambda|x|} \int_{\mathbb{R}^{2}} \frac{1}{2 \pi t \sigma^{2}} \mathrm{e}^{-|x-y|^{2} / 2 t \sigma^{2}} \mathrm{e}^{\lambda|x|-\lambda|y|} d y \\
& \leqslant|\psi|_{\lambda} \phi_{\lambda}(x) \int_{\mathbb{R}^{2}} \frac{1}{2 \pi t \sigma^{2}} \mathrm{e}^{-|x-y|^{2} / 2 t \sigma^{2}} \mathrm{e}^{\lambda|x-y|} d y \\
& =|\psi|_{\lambda} \phi_{\lambda}(x) \int_{\mathbb{R}^{2}} \frac{1}{2 \pi t \sigma^{2}} \mathrm{e}^{-|y|^{2} / 2 t \sigma^{2}} \mathrm{e}^{\lambda|y|} d y,
\end{aligned}
$$

and we are done by definition of $c_{A 1}(t, \lambda)$. 
Lemma A.2. - Assume $\psi \in \mathcal{C}_{\lambda}$ for some $\lambda \in \mathbb{R}$. Then, for any $t>0$, there is a constant $c_{A 2}(t, \lambda)$ such that

$$
\int_{\mathbb{R}^{2}} \mathrm{p}_{t}\left(x-y_{1}\right) \mathrm{p}_{t}\left(x-y_{2}\right) \psi(x) d x \leqslant c_{A 2}(t, \lambda) t^{-1}|\psi|_{\lambda} \phi_{\lambda / 2}\left(y_{1}\right) \phi_{\lambda / 2}\left(y_{2}\right), \quad \forall y_{1}, y_{2} \in \mathbb{R}^{2} .
$$

Proof. - By the Holder inequality, we have

$$
\begin{aligned}
& \int_{\mathbb{R}^{2}} \mathrm{p}_{t}\left(x-y_{1}\right) \mathrm{p}_{t}\left(x-y_{2}\right) \psi(x) d x \\
& \leqslant \sqrt{\int_{\mathbb{R}^{2}} \mathrm{p}_{t}\left(x-y_{1}\right)^{2} \psi(x) d x} \sqrt{\int_{\mathbb{R}^{2}} \mathrm{p}_{t}\left(x-y_{2}\right)^{2} \psi(x) d x} \\
& =c t^{-1} \sqrt{\int_{\mathbb{R}^{2}} \mathrm{p}_{t / 2}\left(x-y_{1}\right) \psi(x) d x} \sqrt{\int_{\mathbb{R}^{2}} \mathrm{p}_{t / 2}\left(x-y_{2}\right) \psi(x) d x} \\
& \leqslant c_{A 2}(t, \lambda) t^{-1}|\psi|_{\lambda} \sqrt{\phi_{\lambda}\left(y_{1}\right) \phi_{\lambda}\left(y_{2}\right)}
\end{aligned}
$$

where the last inequality follows by Lemma A.1.

Lemma A.3. - For any $\lambda, t>0$, set

$$
c_{A 3}(t, \lambda) \equiv \mathrm{e}^{2 t \sigma^{2} \lambda^{2}}, \quad c_{A 4}(t) \equiv \frac{1}{2 \pi t \sigma^{2}} .
$$

Then, for any $\mu \in \mathcal{M}_{\text {rap }}$,

$$
\int \mathrm{p}_{t}(x-y) \mu(d y) \leqslant c_{A 3}(t, \lambda) c_{A 4}(t) \phi_{\lambda}(x) \mu\left(\phi_{-\lambda}\right), \quad \forall \lambda>0, t>0 .
$$

If $\mu \in \mathcal{M}_{\mathrm{tem}}$, then

$$
\int \mathrm{p}_{t}(x-y) \mu(d y) \leqslant c_{A 3}(t, \lambda) c_{A 4}(t) \phi_{-\lambda}(x) \mu\left(\phi_{\lambda}\right), \quad \forall \lambda>0, t>0 .
$$

Proof. - For any $\mu \in \mathcal{M}_{\text {rap }}$

$$
\begin{aligned}
\int \mathrm{p}_{t}(x-y) \mu(d y) & =\frac{1}{2 \pi t \sigma^{2}} \int \mathrm{e}^{-\frac{|x-y|^{2}}{2 t \sigma^{2}}} \mu(d y) \\
& =c_{A 4}(t) \phi_{\lambda}(x) \int \mathrm{e}^{-\frac{|x-y|^{2}-|x| \lambda 2 t \sigma^{2}}{2 t \sigma^{2}}} \mu(d y) \\
& \leqslant c_{A 4}(t) \phi_{\lambda}(x) \int \mathrm{e}^{\frac{\lambda 2 t \sigma^{2}|y|+\lambda^{2}\left(2 t \sigma^{2}\right)^{2}}{2 t \sigma^{2}}} \mu(d y) \\
& =c_{A 3}(t, \lambda) c_{A 4}(t) \phi_{\lambda}(x) \int \mathrm{e}^{\lambda|y|} \mu(d y)
\end{aligned}
$$

where in the third line we used the trivial inequality $a|x|-|x-y|^{2} \leqslant a|y|+a^{2}$ for any $a>0$. (A.3) follows and the proof of (A.4) goes along the same lines. 
Corollary A.4. - For any $\mu \in \mathcal{M}_{\text {rap }}, \lambda>0$ and $0<\varepsilon<T$

$$
\sup _{\varepsilon \leqslant t \leqslant T} \int \mathrm{p}_{t}(x-y) \mu(d y) \leqslant c_{A 3}(T, \lambda) c_{A 4}(\varepsilon) \phi_{\lambda}(x) \mu\left(\phi_{-\lambda}\right) .
$$

For any $\mu \in \mathcal{M}_{\mathrm{tem}}, \lambda>0$ and $0<\varepsilon<T$

$$
\sup _{\varepsilon \leqslant t \leqslant T} \int \mathrm{p}_{t}(x-y) \mu(d y) \leqslant c_{A 3}(T, \lambda) c_{A 4}(\varepsilon) \phi_{-\lambda}(x) \mu\left(\phi_{\lambda}\right) .
$$

Proof. - Immediately from Lemma A.1.

COROLlaRY A.5. - For any $v \in \mathcal{M}_{\text {tem }}$ and $x \in \mathbb{R}^{2}, t \mapsto S_{t} v(x)$ is continuous on $(0, \infty)$

Proof. - By Corollary A.4 with $\mu\left(d y_{1}\right)=\delta_{y}\left(d y_{1}\right)$, for any $0<\varepsilon<T, \lambda>0$,

$$
\begin{aligned}
\sup _{\varepsilon \leqslant t \leqslant T} \mathrm{p}_{t}(x-y) & \leqslant c_{A 3}(T, \lambda) c_{A 4}(\varepsilon) \phi_{-\lambda}(x) \phi_{\lambda}(y) \\
& =c_{A 3}(T, \lambda) c_{A 4}(\varepsilon) \mathrm{e}^{\lambda|x|} \mathrm{e}^{-\lambda|y|}
\end{aligned}
$$

and the result follows by Dominated Convergence since $c_{A 3}(T, \lambda) c_{A 4}(\varepsilon) \mathrm{e}^{\lambda|x|} \times$ $\int_{\mathbb{R}^{2}} \mathrm{e}^{-\lambda|y|} v(d y)<\infty$.

\section{REFERENCES}

[1] M. Barlow, S. Evans, E. Perkins, Collision local times and measure-valued processes, Can. J. Math. 43 (5) (1991) 897-938.

[2] D. Dawson, Measure-valued Markov Processes, École d'été de Probabilités de Saint Flour, 1991.

[3] D. Dawson, A. Etheridge, K. Fleischmann, L. Mytnik, E. Perkins, J. Xiong, Mutually catalytic branching in the plane: Finite measure states, Ann. Probab. 30 (4) (2002) 16811762 .

[4] D. Dawson, A. Etheridge, K. Fleischmann, L. Mytnik, E. Perkins, J. Xiong, Mutually catalytic branching in the plane: infinite measure states, Electron. J. Probab. 7 (15) (2002).

[5] D. Dawson, E. Perkins, Long time behaviour and co-existence in a mutually catalytic branching model, Ann. Probab. 26 (3) (1998) 1088-1138.

[6] P. Donnelly, T. Kurtz, Particle representations for measure-valued population models, Ann. Probab. 27 (1999) 166-205.

[7] S.N. Ethier, T.G. Kurtz, Markov Process: Characterization and Convergence, John Wiley and Sons, New York, 1986.

[8] S. Evans, E. Perkins, Collision local times, historical stochastic calculus, and competing superprocesses, Electron. J. Probab. 3 (5) (1998).

[9] N. Konno, T. Shiga, Stochastic differential equations for some measure-valued diffusions, Probab. Theory Related Fields 79 (1988) 201-225.

[10] P. Meyer, Un cours sur les intégrales stochastiques, in: P. Meyer (Ed.), Séminaire de Probabilités, X, in: Lecture Notes in Mathematics, Vol. 511, Springer, Berlin, 1976, pp. 245-400.

[11] L. Mytnik, Superprocesses in random environments, Ann. Probab. 24 (1996) 1953-1978. 
[12] L. Mytnik, Uniqueness for a mutually catalytic branching model, Probab. Theory Related Fields 112 (2) (1998) 245-253.

[13] E. Perkins, Measure-valued branching diffusions with spatial interactions, Probab. Theory Related Fields 94 (1992) 189-245.

[14] E. Perkins, On the martingale problem for interactive measure-valued branching diffusions, Mem. Amer. Math. Soc. 549 (1995).

[15] M. Reimers, One-dimensional stochastic partial differential equations and the branching measure diffusion, Probab. Theory Related Fields 81 (1989) 319-340.

[16] J. Walsh, An introduction to stochastic partial differential equations, Lecture Notes in Mathematics 1180 (1986) 265-439. 